\title{
A LITTLEWOOD-RICHARDSON RULE FOR TWO-STEP FLAG VARIETIES
}

\author{
IZZET COSKUN
}

\begin{abstract}
This paper studies the geometry of one-parameter specializations of subvarieties of Grassmannians and two-step flag varieties. As a consequence, we obtain a positive, geometric rule for expressing the structure constants of the cohomology of two-step flag varieties in terms of their Schubert basis. A corollary is a positive, geometric rule for computing the structure constants of the small quantum cohomology of Grassmannians. We also obtain a positive, geometric rule for computing the classes of subvarieties of Grassmannians that arise as the projection of the intersection of two Schubert varieties in a partial flag variety. These rules have numerous applications to geometry, representation theory and the theory of symmetric functions.
\end{abstract}

\section{CONTENTs}

1. Introduction

2. Preliminaries

2.1. The cohomology of flag varieties

2.2. Gromov-Witten invariants 6

2.3. Scrolls 6

3. Mondrian tableaux and a Littlewood-Richardson rule for Grassmannians $\quad 7$

$\begin{array}{ll}3.1 . & \text { The rule combinatorially. }\end{array}$

3.2. The geometry of Mondrian tableaux 14

3.3. Amplifications of Theorem $3.32 \quad 20$

4. Painted Mondrian tableaux and a Littlewood-Richardson rule for two-step flag varieties. 24

4.1. Preliminary combinatorial definitions. 24

4.2. The combinatorial Littlewood-Richardson rule. $\quad 27$

4.3. The geometry of painted Mondrian tableau. 39

5. A quantum Littlewood-Richardson rule for Grassmannians 45

$\begin{array}{ll}\text { References } & 46\end{array}$

\section{INTRODUCTION}

This paper studies the geometry of one-parameter specializations of subvarieties of Grassmannians and twostep flag varieties. The first consequence of our study is a new positive, geometric rule for expressing the structure constants of the cohomology ring of Grassmannians in terms of their Schubert basis (Theorem 3.21). Such rules are known as Littlewood-Richardson rules. In fact, much more generally, Algorithm 3.12 provides a positive, geometric algorithm for computing the classes of subvarieties of the Grassmannian that arise as the projection of the intersection of two Schubert varieties in a partial flag variety. The second main application of our study is a positive, geometric rule for computing the structure constants of two-step flag varieties in terms of their Schubert basis (Theorem 4.21). Since the three-pointed Gromov-Witten invariants of a Grassmannian can be calculated as ordinary intersections of Schubert cycles in two-step flag varieties [BKT], we also obtain a quantum Littlewood-Richardson rule for Grassmannians (Theorem 5.1).

2000 Mathematics Subject Classification. Primary 14M15, 14N35, 32M10 .

During the preparation of this article the author was partially supported by a Clay Mathematics Institute Liftoff Fellowship. 
The partial flag variety $F\left(k_{1}, \ldots, k_{r} ; n\right)$ parameterizes nested sequences of $r$ linear subspaces $V_{1}^{k_{1}} \subset \cdots \subset V_{r}^{k_{r}}$ of dimensions $k_{i}$ of a fixed $n$-dimensional vector space $V$. Partial flag varieties are fundamental objects in algebraic geometry, combinatorics and representation theory. Consequently their cohomology rings have been studied extensively (see [BGG], [FPi] or [Ful2]). Although there are many presentations for their cohomology rings, there were no proven Littlewood-Richardson rules for flag varieties except in the case of Grassmannians.

Littlewood-Richardson coefficients exhibit a very rich structure which is best revealed by positive geometric rules. For instance, in recent years, new Littlewood-Richardson rules for the Grassmannians have enabled Klyachko, Knutson, Tao, Woodward and their collaborators to resolve long standing problems such as Horn's Conjecture and the Saturation Conjecture (see $[\mathrm{KT}]$ and $[\mathrm{KTW}]$ ). Vakil using a geometric Littlewood-Richardson rule was able to establish the reality of Schubert calculus [V2].

In the case of Grassmannians there are many Littlewood-Richardson rules. For example, there are LittlewoodRichardson rules in terms of Young tableaux [Ful1], puzzles [KT], and checkers [V1]. A. Knutson conjectured a rule in terms of puzzles (see $([\mathrm{BKT}])$ ) for two-step flag varieties and A. Buch extended the conjecture to three-step flag varieties. However, except for multiplying very special classes (e.g. Monk's formula [Ful1]), a proven rule has eluded mathematicians. Even in the case of Grassmannians, the known rules are insufficient and unsatisfactory for answering many natural geometric questions.

Fix an ordered basis $e_{1}, \ldots, e_{n}$ of a vector space $V$. Let $W_{1}, \ldots, W_{k}$ be $k$ distinct subspaces of $V$ spanned by consecutive elements of the ordered basis. Consider the subvariety $\Sigma$ of $G(k, n)$ parameterizing $k$-planes that intersect each $W_{i}$ in a specified dimension (subject to the necessary compatibility conditions). The first problem we address in this paper is:

Problem 1.1. Give a positive, geometric rule for computing the cohomlogy class of $\Sigma$.

Note that when the vector spaces $W_{i}$ are totally ordered with respect to inclusion, the subvariety $\Sigma$ is a Schubert variety. If the vector spaces $W_{i}$ have no inclusion relations among them, then $\Sigma$ is the intersection of two Schubert varieties in $G(k, n)$. More generally, the projection of the intersection of two Schubert varieties in $F\left(k_{1}, \ldots, k_{r} ; n\right)$ to $G\left(k_{i}, n\right)$ can be described as a variety of the form $\Sigma$. Our first main result is a positive, geometric rule for expressing the class of $\Sigma$ in terms of Schubert cycles.

Theorem 3.32: Algorithm 3.12 gives a positive, geometric rule for computing the class of $\Sigma$.

A corollary of this theorem is a new Littlewood-Richardson rule for Grassmannians. Frank Sottile has pointed out that Algorithm 3.12 and Theorem 3.32 also give a positive, geometric rule for computing Kogan coefficients (See $[\mathrm{BS}],[\mathrm{Kog}])$. I am grateful to Frank Sottile for this valuable observation.

Corollary 1.2 (Frank Sottile). Algorithm 3.12 computes the Kogan coefficients.

The second main result of this paper is a positive geometric rule for computing the structure constants of two-step flag varieties in terms of their Schubert basis.

Theorem 4.21: Algorithm 4.19 is a Littlewood-Richardson rule for two-step flag varieties.

The strategy that leads to the rule works to compute the classes of subvarieties of other partial flag varieties. However, to keep this paper at a reasonable length, we restrict our discussion to two-step flag varieties. The reader may consult [CV] for an example for three-step flag varieties. In addition to being a step in understanding the geometry of arbitrary partial flag varieties, the study of two-step flag varieties is interesting in its own right. For instance, the work of Buch, Kresch and Tamvakis ([BKT]) has highlighted a beautiful relation between the small quantum cohomology of Grassmannians and the cohomology of two-step flag varieties.

The final result of this paper is a quantum Littlewood-Richardson rule for Grassmannians.

Theorem 5.1: Algorithm 4.19 gives a quantum Littlewood-Richardson rule for Grassmannians.

The small quantum cohomology rings of Grassmannians have been studied extensively (see [B], [Bu] or $[\mathrm{BKT}]$ for references). However, as in the case of flag varieties, there was not a positive combinatorial procedure for finding the quantum Littlewood-Richardson coefficients of Grassmannians. Using the results in [BKT], the 
problem of computing three-pointed Gromov-Witten invariants turns to a problem of intersecting three Schubert cycles in a two-step flag variety. Hence, our algorithm also solves the quantum Littlewood-Richardson problem.

Our Littlewood-Richardson rules will be in terms of combinatorial objects called Mondrian tableaux. Mondrian tableaux supply a convenient tool for recording the rank table for the intersections of two flags. We start with the intersection of two Schubert varieties defined with respect to two transverse flags. We specialize the flags via codimension one degenerations. In the process we force the intersection of the two Schubert cycles defined with respect to the two flags to break into a union of Schubert cycles. The limits that occur during this process are again recorded by Mondrian tableaux.

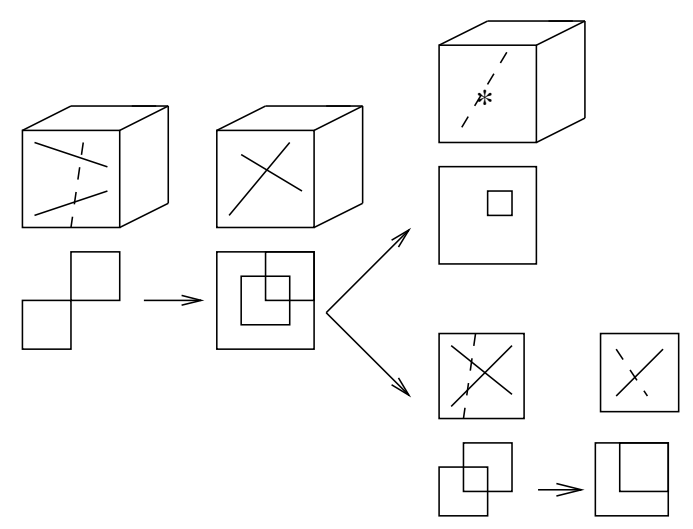

Figure 1. The product $\sigma_{1}^{2}=\sigma_{2}+\sigma_{1,1}$ in $G(2,4)$.

Here we give a simple example of how the rule works. See Figure 1 for geometric pictures and the Mondrian tableaux corresponding to them. Consider the problem of calculating $\sigma_{1}^{2}$ in $G(2,4)$. Geometrically, we would like to compute the class of the locus of lines in $\mathbb{P}^{3}$ that intersect two skew lines $l_{1}$ and $l_{2}$. When $l_{1}$ and $l_{2}$ are skew, describing the lines that intersect both is difficult. Suppose $l_{1}$ and $l_{2}$ intersected at a point. Then the lines that intersect both $l_{1}$ and $l_{2}$ would either contain $l_{1} \cap l_{2}$ or they would be contained in the plane spanned by $l_{1}$ and $l_{2}$. Both of these are Schubert varieties $\left(\sigma_{2}\right.$ and $\sigma_{1,1}$, respectively). To conclude that when the lines are skew that the class is still $\sigma_{2}+\sigma_{1,1}$, we form a one-parameter family of lines by rotating $l_{1}$ around a pivot point until it intersects $l_{2}$. The variety of lines that intersect both $l_{1}$ and $l_{2}$ form a flat family. By the properness of the Hilbert scheme there exists a flat limit when $l_{1}$ and $l_{2}$ intersect. The class of the flat limit is equal to the class of any of the varieties in the family. The flat limit has to be supported along the locus of lines containing $l_{1} \cap l_{2}$ or contained in the span of $l_{1}$ and $l_{2}$. Finally, a (very easy) tangent space calculation shows that the two cycles in the Grassmannian parameterizing lines that intersect $l_{i}$ intersect generically transversally even when $l_{1}$ and $l_{2}$ intersect at a point. It follows that $\sigma_{1}^{2}=\sigma_{2}+\sigma_{1,1}$.

One aim of this paper is to show that this example captures the geometry of Grassmannians. The class of the intersection of two Schubert varieties in the Grassmannian can be calculated (in a sense that will become clear) by judiciously repeating this example. Similarly for two-step flag varieties there are three simple examples that capture the geometry. The first is the calculation

$$
\sigma_{1,0,0}^{2,1,2} \cdot \sigma_{1,1,0}^{2,1,2}=\sigma_{2,2,0}^{2,1,2}+\sigma_{2,1,0}^{1,2,2}+\sigma_{1,1,1}^{1,2,2}
$$

in $F(1,3 ; 5)$. The left-hand side of Figure 2 depicts this calculation. Geometrically this calculation corresponds to finding the class of pointed planes $(p, P)$ in $\mathbb{P}^{4}$ where the point intersects a line $l_{2}$ (drawn as the red square) and the plane contains the point and intersects the lines $l_{1}$ and $l_{3}$. We rotate $l_{1}$ until it intersects $l_{2}$. Either the plane $P$ intersects $l_{1} \cap l_{2}$, but the point $p$ is distinct from $l_{1} \cap l_{2}$. In that case $P$ must contain $l_{2}$ and we obtain the Schubert variety $\sigma_{2,2,0}^{2,1,2}$. Or the point $p$ might coincide with $l_{1} \cap l_{2}$. In this case, we get the Schubert variety $\sigma_{2,1,0}^{1,2,2}$. Finally, if neither the plane $P$ nor the point $p$ intersects $l_{1} \cap l_{2}$, then they have to be contained in the new span of $l_{1}, l_{2}$ and $l_{3}$. In this case, we get the Schubert variety $\sigma_{1,1,1}^{1,2,2}$. 


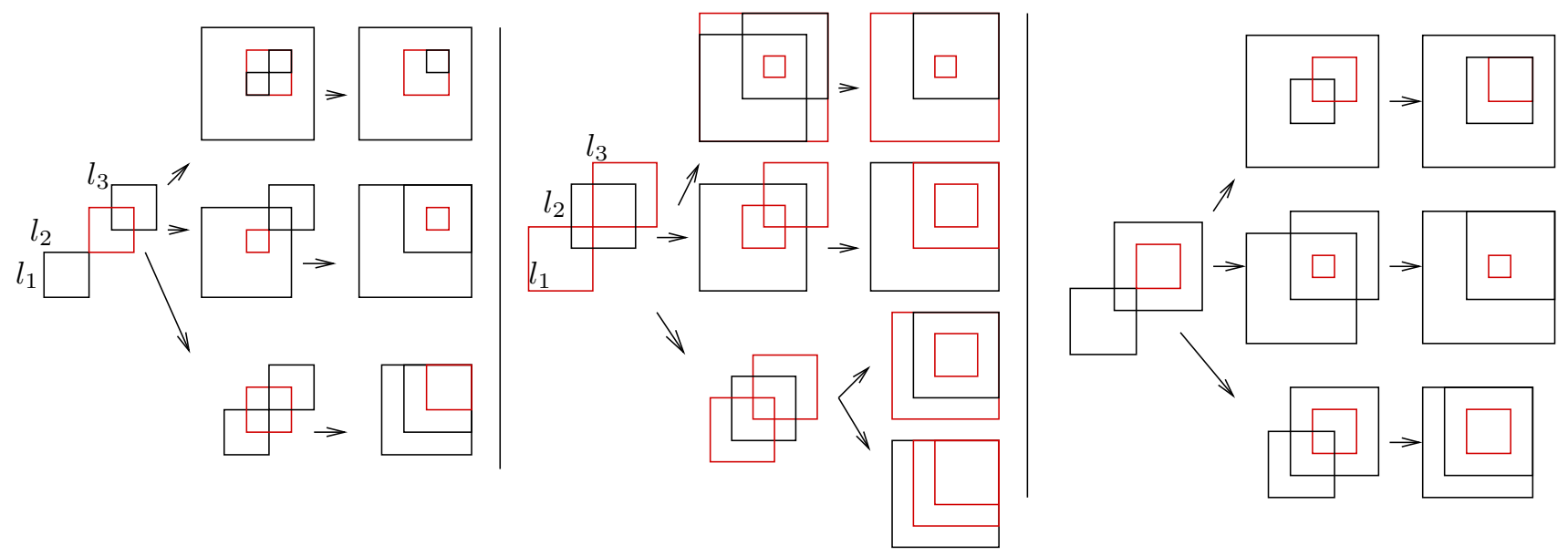

Figure 2. Two calculations in two-step flag varieties.

The second calculation is

$$
\sigma_{1,0,0}^{1,2,1} \cdot \sigma_{1,1,0}^{1,2,1}=\sigma_{3,1,0}^{1,2,1}+\sigma_{2,1,0}^{1,1,2}+\sigma_{2,1,1}^{1,2,1}+\sigma_{1,1,1}^{1,1,2}
$$

in $F(2,3 ; 6)$. This calculation is depicted in the middle of Figure 2. Geometrically this calculation corresponds to finding the class of line and plane pairs $(l, P)$ in $\mathbb{P}^{5}$, where the line $l$ intersects two planes $l_{1}$ and $l_{3}$ and the plane $P$ contains $l$ and intersects the plane $l_{2}$. We rotate $l_{1}$ until it intersects $l_{2}$. The possible limits in this case are similar to the previous examples. Either $l$ intersects $l_{1} \cap l_{3}$. In this case we get the Schubert variety $\sigma_{3,1,0}^{1,2,1}$. Or $P$ may intersect $l_{1} \cap l_{2}$. In this case we get the Schubert variety $\sigma_{2,1,0}^{1,1,2}$. Else both $l$ and $P$ have to be contained in the new span of $l_{1}, l_{2}$ and $l_{3}$. The last variety is not a Schubert variety, but by increasing the intersection of $l_{1}$ and $l_{2}$ by one more dimension is easily seen to break into a sum of two Schubert varieties $\sigma_{2,1,1}^{1,2,1}+\sigma_{1,1,1}^{1,1,2}$. Finally, the third calculation is

$$
\sigma_{1,0,0}^{2,1,2} \cdot \sigma_{1,1,0}^{1,2,2}=\sigma_{3,1,0}^{1,2,2}+\sigma_{2,2,0}^{1,2,2}+\sigma_{2,1,1}^{1,2,2} .
$$

This example is represented on the left panel of Figure 2 and is similar to the previous ones.

Algorithms 3.12 and 4.19 are generalizations of these simple examples. We will degenerate the flags that define the intersection of two Schubert varieties via specified codimension one degenerations. The flat limits are determined by the following observation.

Observation 1.3. Suppose $\Lambda$ is a $k$-plane that intersects a set of $k$-vector spaces $W_{i}$ in specified dimensions $d_{i}$. Then $\Lambda$ will be forced to intersect the spans of $W_{i_{1}}, \ldots, W_{i_{j}}$ in given dimensions $d_{i_{1}, \ldots, i_{j}}$. Consider a codimension one specialization of the vector spaces $W_{i}$. Then any limit of $\Lambda$ has to intersect the limits of the vector spaces $W_{i}$ in dimension at least $d_{i}$. Similarly, $\Lambda$ has to intersect the limits of the spans of $W_{i_{1}}, \ldots, W_{i_{j}}$ in dimension at least $d_{i_{1}, \ldots, i_{j}}$.

Theorem 3.32 and Theorem 4.45 will check that this observation suffices to determine the set-theoretic components of the flat limit of codimension one degenerations in Grassmannians and two-step flag varieties. As a matter of fact, the same observation applies to arbitrary partial flag varieties and can be used to calculate the classes of subvarieties in them. Theorems 3.32 and 4.45 will also show that for the specified degenerations each of the components occur with multiplicity one.

The idea to study the geometry of flag varieties via degenerations dates back at least to Pieri. Recently R. Vakil proved a geometric Littlewood-Richardson rule for Grassmannians using degenerations [V1]. Our approach is similar to Vakil's. However, even in the case of Grassmannians, by choosing a more natural and canonical degeneration order, we are able to clarify the geometry significantly. The result is a simpler and more efficient rule. In fact, as already mentioned, our methods apply in a much more general setting and give an algorithm to express the classes of a large collection of subvarieties of Grassmannians in terms of Schubert cycles. 
We remark that the positive, geometric rules proved in this paper have many applications to reality questions, Schubert calculus over fields other than complex numbers and saturation type conjectures. To keep this paper relatively short, we postpone the discussion of these applications to a future paper.

Finally, it should be stressed that the algorithms described in this paper do not only compute classes of subvarieties of Grassmannians or two-step flag varieties. They describe the limits of subvarieties of Grassmannians and two-step flag varieties under specializations of the defining flags. The end result of the algorithm is not a collection of cohomology classes, but actual subvarieties.

Acknowledgments: I would like to thank C. Desjardins, S. Grushevsky, J. Harris, A. Knutson, A. Kresch, B. Osserman, F. Sottile and H. Tamvakis for fruitful conversations. I am grateful to F. Sottile for pointing out Corollary 1.2. I am greatly indebted to R. Vakil and A. Buch for their constant help and unfailing support. They have generously volunteered their time and effort to implement the algorithms in this paper. Their suggestions have greatly improved the accuracy, content and presentation of this paper.

\section{Preliminaries}

In this section we collect well-known facts about flag varieties and the quantum cohomology of Grassmannians. For more detailed discussions the reader can consult [GH] Ch.1 §5, [FPi] and [BKT].

Notation 2.1. Let $k_{i}$ be an increasing sequence of positive integers. We denote the Grassmannian of $k$ dimensional subspaces of an $n$-dimensional vector space by $G(k, n)$. When we interpret this Grassmannian as the parameter space of $(k-1)$-dimensional linear subspaces of $\mathbb{P}^{n-1}$, we will use the notation $\mathbb{G}(k-1, n-1)$.

Let $F\left(k_{1}, \ldots, k_{r} ; n\right)$ denote the $r$-step flag variety of $r$-tuples of linear subspaces $\left(V_{1}, \cdots, V_{r}\right)$ of an $n$ dimensional fixed vector space, where $V_{i}$ are $k_{i}$-dimensional linear spaces and $V_{i} \subset V_{i+1}$ for all $1 \leq i \leq r-1$. When we would like to consider the flag variety as a parameter space for nested sequences of linear subspaces of projective space, we will use the notation $\mathbb{F}\left(k_{1}-1, \ldots, k_{r}-1 ; n-1\right)$.

2.1. The cohomology of flag varieties. Let $F_{\bullet}: 0=F_{0} \subset F_{1} \subset \cdots \subset F_{n}=V$ be a fixed complete flag in the $n$-dimensional vector space $V$. Given a partition $\lambda: n-k \geq \lambda_{1} \geq \lambda_{2} \geq \cdots \geq \lambda_{k} \geq 0$, we define the Schubert variety $\Sigma_{\lambda}$ in $G(k, n)$ with respect to the flag $F_{\bullet}$ to be

$$
\Sigma_{\lambda}\left(F_{\bullet}\right):=\left\{[W] \in G(k, n) \mid \operatorname{dim}\left(W \cap F_{n-k+i-\lambda_{i}}\right) \geq i, \forall 1 \leq i \leq k\right\} .
$$

We denote the Poincaré dual of the class of $\Sigma_{\lambda}$ by $\sigma_{\lambda}$. The codimension of $\Sigma_{\lambda}$ in $G(k, n)$ is equal to the weight of the partition $|\lambda|=\sum_{i} \lambda_{i}$. The classes of Schubert varieties form an additive basis of the homology of $G(k, n)$. The structure constants $c_{\lambda, \mu}^{\nu}$ in the product $\sigma_{\lambda} \cdot \sigma_{\mu}=\sum_{\nu} c_{\lambda, \mu}^{\nu} \sigma_{\nu}$ are called the Littlewood-Richardson coefficients.

Similarly, the cohomology of the $r$-step flag variety $F\left(k_{1}, \ldots, k_{r} ; n\right)$ is generated by Poincaré duals of the classes of Schubert varieties. Schubert varieties are parameterized by permutations $\omega$ of length $n$ for which $\omega(i)<\omega(i+1)$ whenever $i \notin\left\{k_{1}, \ldots, k_{r}\right\}$. More explicitly, the Schubert variety $X_{\omega}\left(F_{\bullet}\right)$ is defined by

$$
X_{\omega}\left(F_{\bullet}\right):=\left\{\left(V_{1}, \ldots, V_{r}\right) \in F\left(k_{1}, k_{2} ; n\right) \mid \operatorname{dim}\left(V_{i} \cap F_{j}\right) \geq \#\{\alpha \leq i: \omega(\alpha)>n-j\} \forall i, j\right\}
$$

The Poincaré duals of the classes of all the Schubert varieties form an additive basis for the cohomology of the flag variety. The structure constants with respect to this basis are known as the Littlewood-Richardson coefficients for flag varieties.

For future reference we note that given a Schubert cycle $\sigma_{\lambda}$ in $G(k, n)$, there is a special Schubert cycle $X_{\lambda}^{(d)}\left(F_{\bullet}\right)$ in $F(k-d, k+d ; n)$ defined by

$$
X_{\lambda}^{(d)}\left(F_{\bullet}\right):=\left\{\left(V_{1}, V_{2}\right) \mid \operatorname{dim}\left(V_{1} \cap F_{n-i-\lambda_{k-i}}\right) \geq k-d-i, \operatorname{dim}\left(V_{2} \cap F_{n-k+j-\lambda_{j}}\right) \geq j\right\}
$$

where $1 \leq i \leq k-d$ and $1 \leq j \leq k$.

We need a convenient notation for Schubert varieties of $r$-step flag varieties. In analogy with the notation for the Grassmannians we will use the notation $\sigma_{\lambda_{1}, \cdots, \lambda_{k_{r}}}^{\delta_{1}, \cdots, \delta_{k_{r}}}$. The bottom row denotes the usual partition corresponding to the $k_{r}$-plane $V_{r}$ in $V$ treated as a Schubert cycle in $G\left(k_{r}, n\right)$. The numbers $\delta_{i}$ are integers between 1 and $r$. For a Schubert cycle in $F\left(k_{1}, \ldots, k_{r} ; n\right), k_{1}$ of the upper indices will be 1 and $k_{i}-k_{i-1}$ of them will 
be $i$. The flag $F_{\bullet}$ induces a complete flag $G_{\bullet}$ on the largest vector space $V_{r}$. For each $j$, there exists a smallest $i$ such that

$$
\operatorname{dim}\left(V_{i} \cap G_{j}\right)=\operatorname{dim}\left(V_{i} \cap G_{j-1}\right)+1 .
$$

For a Zariski-open subset of the Schubert variety this index will be constant. In that case we write $i$ on top of $\lambda_{j}$. In the case of Grassmannians this notation reduces to the ordinary notation with a sequence of 1 s on the top row. For the complete flag variety the top row becomes the permutation defining the Schubert cycle.

Example. Fix a flag $F_{1} \subset \cdots \subset F_{6}$ in $V^{6}$. The Schubert cycle $\sigma_{3,2}$ in $G(2,6)$ denotes the two-dimensional subspaces of $V^{6}$ that meet $F_{2}$ in a line and are contained in $F_{4}$. The corresponding special Schubert cycle $X_{\sigma_{3,2}}^{1}$ has class $\sigma_{2,1,0}^{2,1,2}$ in $F(1,3 ; 6)$. The Schubert cycle $\sigma_{2,1,0}^{2,1,2}$ denotes the pairs of subspaces $V_{1} \subset V_{2}$ where $V_{1}$ has dimension one and $V_{2}$ has dimension $3 . V_{2}$ is required to meet $F_{2}$ in dimension one, $F_{4}$ in dimension 2 and be contained in $F_{6}$. $V_{1}$ lies in the intersection of $V_{2}$ with $F_{4}$.

Remark 2.2. Sometimes Schubert cycles in $F\left(k_{1}, \ldots, k_{r} ; n\right)$ are denoted by strings of $0,1, \ldots, r$ of length $n$ where $k_{1}$ of the numbers in the string are $1, k_{i}-k_{i-1}$ of them are $i$ and $n-k_{r}$ of them are 0 . The translation from our notation to this notation is straightforward. Place a 0 in a position unless it is of the form $n-k_{r}+i-\lambda_{i}$. Place the digit $\delta_{i}$ in position $n-k_{r}+i-\lambda_{i}$. We warn the reader that there are different conventions for the string notation. Some authors use a different correspondence between the digits and the subscripts of the vector spaces. Similarly there are different conventions as to whether the string should be written from left to right or right to left.

2.2. Gromov-Witten invariants. Let $\bar{M}_{0, m}(\mathbb{G}(k, n), d)$ denote the Kontsevich space of genus zero stable maps to $\mathbb{G}(k, n)$ of Plücker degree $d$. The Kontsevich space is equipped with $m$ evaluation morphisms,

$$
\rho_{1}, \ldots, \rho_{m}: \bar{M}_{0, m}(\mathbb{G}(k, n), d) \rightarrow \mathbb{G}(k, n),
$$

where the $i$-th evaluation morphism maps a stable map to the image of the $i$-th marked point.

Given $m$ Schubert classes $\sigma_{\lambda_{1}}, \cdots, \sigma_{\lambda_{m}}$ in $\mathbb{G}(k, n)$, the Gromov-Witten invariant $I_{d}\left(\sigma_{\lambda_{1}}, \ldots, \sigma_{\lambda_{m}}\right)$ is defined by the formula

$$
I_{d}\left(\sigma_{\lambda_{1}}, \ldots, \sigma_{\lambda_{m}}\right)=\int_{\bar{M}_{0, m}(\mathbb{G}(k, n), d)} \rho_{1}^{*}\left(\sigma_{\lambda_{1}}\right) \cup \cdots \cup \rho_{m}^{*}\left(\sigma_{\lambda_{m}}\right) .
$$

Since the three-pointed Gromov-Witten invariants $(m=3)$ give the structure constants of the small quantum cohomology ring, they are called the quantum Littlewood-Richardson coefficients.

Let $\Sigma_{1}, \cdots, \Sigma_{m}$ be general (with respect to the $\mathbb{P} G L(n+1)$ action) Schubert cycles representing the Poincaré duals of the classes $\sigma_{\lambda_{1}}, \ldots, \sigma_{\lambda_{m}}$, respectively. The following lemma asserts that the Gromov-Witten invariant is equal to the number of rational curves that intersect $\Sigma_{i}$.

Lemma 2.3. ([FP] Lemma 14) The scheme theoretic intersection

$$
\rho_{1}^{-1}\left(\Sigma_{1}\right) \cap \cdots \cap \rho_{m}^{-1}\left(\Sigma_{m}\right)
$$

is a finite number of reduced points in $M_{0, m}(\mathbb{G}(k, n), d)$. Moreover,

$$
I_{d}\left(\sigma_{\lambda_{1}}, \ldots, \sigma_{\lambda_{m}}\right)=\# \rho_{1}^{-1}\left(\Sigma_{1}\right) \cap \cdots \cap \rho_{m}^{-1}\left(\Sigma_{m}\right)
$$

Remark 2.4. Furthermore, by Kleiman's Transversality Theorem [Kl] one can conclude that the curves contributing to the Gromov-Witten invariants are non-degenerate curves and the restriction of the tautological bundle of $\mathbb{G}(k, n)$ to the curves have balanced splitting (i.e. the degree of any two summands in the Grothendieck decomposition differ by at most one).

2.3. Scrolls. Let $r_{1} \leq \cdots \leq r_{k}$ be non-negative integers, not all equal to zero. We let $r$ be the sum $\sum_{i=1}^{k} r_{i}$. Let $S_{r_{1}, \cdots, r_{k}}$ denote the $k$-dimensional rational normal scroll in $\mathbb{P}^{r+k-1}$.

To construct it take $k$ rational normal curves of degree $r_{1}, \ldots, r_{k}$ in $\mathbb{P}^{r+k-1}$ such that the span of any $k-1$ of them is disjoint from the span of the remaining one. Fix an isomorphism between each of these curves and an abstract $\mathbb{P}^{1} . S_{r_{1}, \cdots, r_{k}}$ is the union of the $(k-1)$-planes spanned by the points corresponding under the isomorphism. We allow some of the integers $r_{i}$ to be zero. In that case we obtain cones over smaller dimensional scrolls. A scroll is balanced if $\left|r_{i}-r_{j}\right| \leq 1$ for $1 \leq i, j \leq k$. It is perfectly balanced if all $r_{i}$ are equal. 
Abstractly a scroll is the projectivization of a vector bundle of rank $k$ on $\mathbb{P}^{1}$. Since any vector bundle $E$ over $\mathbb{P}^{1}$ is a sum of line bundles, we can express the projectivization as

$$
\mathbb{P} E=\mathbb{P}\left(\mathcal{O}_{\mathbb{P}^{1}}\left(-r_{1}\right) \oplus \cdots \oplus \mathcal{O}_{\mathbb{P}^{1}}\left(-r_{k}\right)\right) .
$$

The scroll $S_{r_{1}, \cdots, r_{k}}$ is the image of $\mathbb{P} E$ under the linear series $\left|\mathcal{O}_{\mathbb{P} E}(1)\right|$.

Scrolls as rational curves in the Grassmannian. Since a scroll $S_{r_{1}, \cdots, r_{k}}$ is a family of $(k-1)$-planes parameterized by a rational curve, it gives rise to a rational curve $C$ in $\mathbb{G}(k-1, r+k-1)$. The curve $C$ is non-degenerate (i.e., does not lie in a subgrassmannian of the form $\mathbb{G}(k-1, s)$ for $s<r+k-1)$ and of Plücker degree $r=\sum r_{i}$.

Conversely, any irreducible, non-degenerate curve $C$ in the Grassmannian $\mathbb{G}(k-1, r+k-1)$ of degree $r$ gives rise to a scroll of degree $r$ in $\mathbb{P}^{r+k-1}$. There are non-isomorphic scrolls of degree $r$ in $\mathbb{P}^{r+k-1}$. The splitting type of the restriction of the tautological bundle of $\mathbb{G}(k-1, r+k-1)$ to $C$ determines the isomorphism type of the scroll (see $[\mathrm{C}]$ ). We, therefore, obtain the following corollary of Lemma 2.3 and Remark 2.4.

Corollary 2.5. The Gromov-Witten invariant $I_{d}\left(\sigma_{\lambda_{1}}, \ldots, \sigma_{\lambda_{m}}\right)$, where $\sigma_{\lambda_{1}}, \ldots, \sigma_{\lambda_{m}}$ are Schubert cycles in $G(k, n)$, is equal to the number of balanced scrolls of degree $d$ and dimension $d+k-1$ in $\mathbb{P}^{n-1}$ containing $m$ fibers satisfying the specified Schubert conditions.

In $[\mathrm{BKT}]$ the authors relate the quantum three-point invariants of degree $d$ for $G(k, n)$ to ordinary intersections in $F(k-d, k+d ; n)$. Since we will use their Proposition 1 and Corollary 1 , we summarize their results in terms of the geometric point of view offered by Corollary 2.5.

Lemma 2.6. ([BKT] Prop.1) The only non-zero three-point quantum invariant of $G(d, 2 d)$ of degree $d$ is

$$
I_{d}\left(\sigma_{d, \cdots, d}, \sigma_{d, \cdots, d}, \sigma_{d, \cdots, d}\right)=1
$$

By dimension restrictions, the stated invariant is the only non-zero degree $d$ invariant. The invariant is equal to one because there is a unique scroll of degree and dimension $d$ in $\mathbb{P}^{2 d-1}$ containing three general $(d-1)$ dimensional linear spaces $A, B, C$ as fibers. In such a scroll, through every point $p$ of $A$ there is a unique line meeting $B$ and $C$. This line is $\overline{p B} \cap \overline{p C}$, the intersections of the span of $p$ and $B$ and the span of $p$ and $C$. This uniquely constructs the scroll.

Lemma 2.7. ([BKT] Cor.1) Let $\lambda, \mu, \nu$ be partitions and $d \geq 0$ be an integer satisfying

$$
|\lambda|+|\mu|+|\nu|=k(n-k)+d n .
$$

Then the degree $d$ three-point Gromov-Witten invariants of $G(k, n)$ equal the ordinary three-point intersections of special Schubert varieties (see §2.1) in the flag variety $F(k-d, k+d ; n)$ :

$$
I_{d}\left(\sigma_{\lambda}, \sigma_{\mu}, \sigma_{\nu}\right)=\int_{F(k-d, k+d ; n)}\left[X_{\lambda}^{(d)}\right] \cup\left[X_{\mu}^{(d)}\right] \cup\left[X_{\nu}^{(d)}\right] .
$$

By Corollary 2.5 the Gromov-Witten invariant is equal to the number of balanced scrolls of degree $d$ and dimension $k$ in $\mathbb{P}^{n-1}$. Such a scroll is a cone over a perfectly balanced scroll with vertex a linear space of dimension $k-d-1$. It spans a $\mathbb{P}^{k+d-1}$. To each scroll contributing to the Gromov-Witten invariant one associates the pair of linear spaces consisting of the vertex and the span of the cone. This gives the required bijection.

\section{Mondrian tableaux And a Littlewood-Richardson Rule For Grassmannians}

In this section we describe a way to compute the Littlewood-Richardson coefficients of Grassmannians in terms of combinatorial objects called Mondrian tableaux. In fact, we will give a geometric rule for computing the classes of a much larger class of subvarieties of $G(k, n)$. We will first give the combinatorial description of the rule. We will then explain the geometry behind the rule. We will then present some generalizations that are useful for studying the geometry of two-step flag varieties.

\subsection{The rule combinatorially.}


3.1.1. Preliminaries. Fix $n$ basis elements $e_{1}, \ldots, e_{n}$ ordered by their indices. A square is a subset of the set of basis elements. The side-length of a square $S$ is the number of basis elements contained in $S$. We will denote the side-length of $S$ by $|S|$. Let $M$ be a set of squares and let $S$ be any square (not necessarily contained in $M)$. We will denote by $\# S(M)$, the number of squares of $M$ (inclusive) contained in $S$.

Definition 3.1. A Mondrian tableau $M$ for the Grassmannian $G(k, n)$ is a collection of $k$ distinct squares such that

Mon1 Each square is the span of a subset of consecutive basis elements of $\left\{e_{1}, \ldots, e_{n}\right\}$.

Mon2 For each square $S$, the union of the squares strictly contained in $S$ is properly contained in $S$.

Mon3 For any two squares $S_{1}, S_{2}$ of $M$,

$$
\left|S_{1} \cup S_{2}\right| \geq\left|S_{1}\right|+\#\left(S_{1} \cup S_{2}\right)(M)-\# S_{1}(M) .
$$

We will depict a Mondrian tableau by placing the basis elements as unit squares along the diagonal of an $n \times n$ grid listed from southwest to northeast. A square $S$ in the Mondrian tableau will be depicted by a square whose diagonal lies along the diagonal of the $n \times n$ grid and contains the unit squares corresponding to the basis elements contained in $S$. Figure 3 depicts some Mondrian tableaux.

Some examples of Mondrian Tableaux

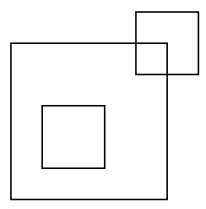

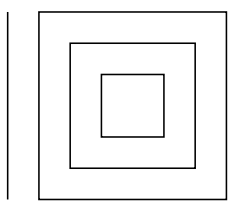

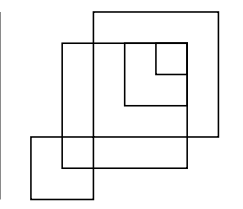

Some non-examples

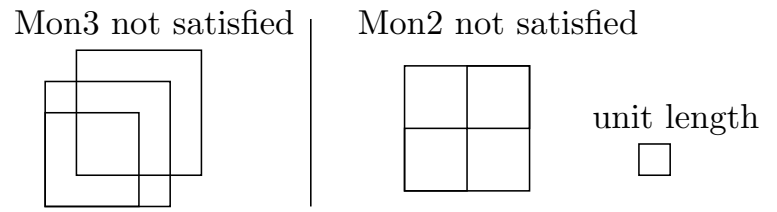

Figure 3. Some examples and non-examples of Mondrian tableaux.

Remark 3.2. Condition Mon3 implies the following a priori stronger consistency condition: Let $S_{1}, \ldots, S_{j}$ be any set of squares in a Mondrian tableau $M$. Then

$$
\left|\bigcup_{l=1}^{j} S_{l}\right| \geq\left|S_{i}\right|+\#\left(\bigcup_{l=1}^{j} S_{l}\right)(M)-\# S_{i}(M) .
$$

A Mondrian tableau $M$ for $G(k, n)$ represents an irreducible subvariety $\Sigma_{M}$ of $G(k, n)$. In Section 3.2 we will explain how to associate a subvariety of $G(k, n)$ to a Mondrian tableau. Briefly, the squares of a Mondrian tableau $M$ represent vector spaces that impose rank conditions on $k$-planes. The subvariety $\Sigma_{M}$ is the closure of the locus of $k$-planes that intersect the vector space spanned by the basis elements contained in any square $S$ in dimension \# $\#(M)$. We will denote the Poincaré dual of the class of $\Sigma_{M}$ by $\left[\Sigma_{M}\right]$. Below we will give an algorithm for expressing $\left[\Sigma_{M}\right]$ in terms of Schubert cycles.

3.1.2. Representing Schubert varieties as Mondrian tableaux. We now describe how to represent Schubert varieties by Mondrian tableaux.

Definition 3.3. A collection of squares $S_{1}, \ldots, S_{r}$ in a Mondrian tableau are called nested if the squares are totally ordered with respect to inclusion; i.e., for any two squares $S_{i}$ and $S_{j}$ either $S_{i} \subseteq S_{j}$ or $S_{j} \subseteq S_{i}$ for every $1 \leq i, j \leq r$. We will say a square $S$ in a Mondrian tableau $M$ is nested if

(1) For any other square $S^{\prime}$ in $M$ either $S \subset S^{\prime}$ or $S^{\prime} \subset S$.

(2) The collection of all the squares in $M$ containing $S$ is nested.

We will say that a Mondrian tableau is nested if all the squares in $M$ are nested.

Definition 3.4. A Mondrian tableau associated to a Schubert variety $\sigma_{\lambda_{1}, \ldots, \lambda_{k}}$ in $G(k, n)$ is a nested Mondrian tableau $S_{1} \subset S_{2} \subset \cdots \subset S_{k}$ where the side-length $\left|S_{i}\right|=n-k+i-\lambda_{i}$. 
Figure 4 depicts two Mondrian tableaux for $\sigma_{2,1}$ in $G(3,6)$. These Mondrian tableaux represent the same Schubert class, but different Schubert varieties.

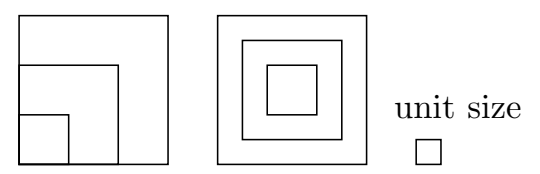

Figure 4. Two Mondrian tableaux associated to $\sigma_{2,1}$ in $G(3,6)$.

Definition 3.5. The lower-left (respectively, upper-right) corner of a square $S$ in a Mondrian tableau $M$ is the basis element with least (respectively, greatest) index contained in $S$. We will denote the lower-left corner of $S$ by $l(S)$ and the upper-right corner of $S$ by $r(S)$. We will say $e_{i}$ is southwest (respectively, northeast) of $e_{j}$ if $i \leq j$ (respectively $i \geq j$ ). We will say strictly southwest or northeast to mean the inequalities are strict.

Definition 3.6 (Normalized Mondrian tableau). A Mondrian tableau $M$ is called normalized if

(1) The lower-left corners of any two squares in $M$ are distinct.

(2) If the upper-right corners of two distinct squares $S_{1} \subset S_{2}$ coincide, then any square $S \in M$ with $l(S)<l\left(S_{2}\right)$ contains $S_{1}$.

Remark 3.7. Let $M$ be a set of squares satisfying condition Mon1. If the lower-left (or upper-right) corners of all the squares are distinct, then condition Mon2 automatically holds. This is clear since for any square $S, l(S)$ (or $r(S)$ ) cannot be contained in any of the squares strictly contained in $S$. Furthermore, if $M$ is normalized, then Mon3 automatically holds. Let $S_{1}$ and $S_{2}$ be any two squares of $M$. If $l\left(S_{1}\right)<l\left(S_{2}\right)$ and $r\left(S_{1}\right)<r\left(S_{2}\right)$, then $\#\left(S_{1} \cup S_{2}\right)(M)-\# S_{2}(M)$ (respectively, \# $\left(S_{1} \cup S_{2}\right)(M)-\# S_{1}(M)$ ) is at most $\left|S_{1}-S_{2}\right|$ (respectively, $\left.\left|S_{2}-S_{1}\right|\right)$. In the first case (respectively, second case), each square contributing to the count has a distinct lower-left (respectively, upper-right) corner. If $S_{2} \subset S_{1}$, then $\#\left(S_{1} \cup S_{2}\right)(M)-\# S_{2}(M)$ is at most $\left|S_{1}-S_{2}\right|$ since each square contributing to the count occupies either a distinct upper-right or lower-left corner not contained in $S_{2}$.

3.1.3. The algorithm for normalizing a collection of squares. Let $M$ be a finite collection of squares satisfying condition Mon1. If for $S_{1}, S_{2} \in M$, the lower-left (respectively, upper-right) corners $l\left(S_{1}\right)=l\left(S_{2}\right)$ (respectively, $\left.r\left(S_{1}\right)=r\left(S_{2}\right)\right)$ coincide, then either $S_{1} \subseteq S_{2}$ or $S_{2} \subseteq S_{1}$. We can normalize this collection of squares. In case $M$ is not a Mondrian tableau, it is possible that the procedure yields the empty collection. We will say discard the tableau to refer to the latter possibility.

Algorithm 3.8 (Normalizing a collection of squares). Suppose there exists a basis element which is the lower-left corner of more than one square. Let $e_{i}$ be the one with least index. Let $S_{1} \subseteq S_{2} \subseteq \cdots \subseteq S_{r}$ be the squares in $M$ that have $e_{i}$ as the lower-left corner. (If some of the squares in the collection coincide, pick any ordering on them. Otherwise order them by size.) Remove $e_{i}$ from $S_{2}$ and call the resulting square $S_{2}^{\prime}$. (Pictorially this corresponds to shrinking the square $S_{2}$ by moving $l\left(S_{2}\right)$ northeast one unit.) Replace $M$ by the new collection of squares $M^{\prime}$ obtained by replacing $S_{2}$ by $S_{2}^{\prime}$. If no two squares of $M^{\prime}$ share the same lower-left corner, stop. Otherwise run the procedure again, replacing $M$ by $M^{\prime}$. If the side-length of any square shrinks to zero in the process, discard the collection of squares.

After finitely many steps we will obtain a collection of squares $M$ that do not share the same lower-left corner. If $M$ is not normalized, then there exists two squares $S_{1} \subset S_{2}$ such that $S_{1} \subset S_{2}, r\left(S_{1}\right)=r\left(S_{2}\right)$ and violate Condition 2 in Definition 3.6. Let $e_{i}$ be the basis element with largest index which is the upper-right corner of such squares. Let $S_{1} \subset S_{2} \subset \cdots \subset S_{r}$ be the squares with $r\left(S_{j}\right)=e_{i}$ and which violate Condition 2 in Definition 3.6. Remove $e_{i}$ from $S_{2}$ call the resulting square $S_{2}^{\prime}$. Replace $M$ by the new collection $M^{\prime}$ obtained by replacing $S_{2}$ by $S_{2}^{\prime}$. If $M^{\prime}$ is normalized, stop. Otherwise, repeat by replacing $M$ by $M^{\prime}$. If the side-length of any square shrinks to zero in the process, discard the collection of squares.

Figure 5 shows an example of the Algorithm 3.8 applied to a Mondrian tableau. 


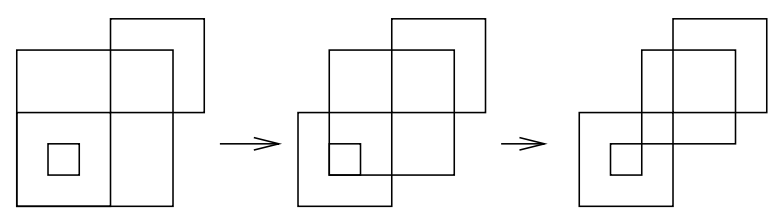

FIGURE 5. The normalization algorithm applied to a Mondrian tableau.

Lemma 3.9. The algorithm for normalizing a collection of squares transforms a Mondrian tableau for $G(k, n)$ to a normalized Mondrian tableau for $G(k, n)$.

Proof. It is clear that the algorithm terminates. We need to check that at each stage the procedure transforms a Mondrian tableau to a Mondrian tableau. Here we check the case when we shrink the lower-left corner of a square. The case when we shrink the upper-right corner is analogous. Mon1 holds since $S_{2}^{\prime}$ is still the span of consecutive basis elements and the other squares do not change. Note that if $S_{2}^{\prime}$ has the same lower-left corner as a different square $S$ in $M^{\prime}$, then $S \subset S_{2}^{\prime}$. Otherwise, $S_{2}^{\prime} \subset S$, but $|S|=\left|S_{2} \cup S\right|-1$ contrary to Mon3 for $M$ since $S_{1}$ and $S_{2}$ are not contained in $S$. Hence, all the containment relations that hold in $M$ except for $S_{1} \subset S_{2}$ hold for $M^{\prime}$ when $S_{2}$ is replaced by $S_{2}^{\prime}$. Furthermore, there are no new containment relations among the squares in $M^{\prime}$. We only need to check the conditions Mon2 and Mon3 for $S_{2}^{\prime}$. $S_{2}^{\prime}$ cannot be the span of squares contained in it, otherwise $S_{2}$ would be as well. This would contradict Mon2 for $M$. Finally, suppose $S$ is a square of $M^{\prime}$ such that

i) $\left|S \cup S_{2}^{\prime}\right|<|S|+\#\left(S \cup S_{2}^{\prime}\right)\left(M^{\prime}\right)-\# S\left(M^{\prime}\right)$, or

ii) $\left|S \cup S_{2}^{\prime}\right|<\left|S_{2}^{\prime}\right|+\#\left(S \cup S_{2}^{\prime}\right)\left(M^{\prime}\right)-\# S_{2}^{\prime}\left(M^{\prime}\right)$.

We have that a) $\left|S \cup S_{2}^{\prime}\right|=\left|S \cup S_{2}\right|$ unless $e_{i} \notin S$ and b) $\left|S \cup S_{2}^{\prime}\right|=\left|S \cup S_{2}\right|-1$. If we are in case i and a, then

$$
\left|S \cup S_{2}\right|<|S|+\#\left(S \cup S_{2}\right)(M)-\# S(M) .
$$

If we are in case $\mathrm{i}$ and $\mathrm{b}$, then

$$
\left|S \cup S_{2}\right|-1<|S|+\#\left(S \cup S_{2}\right)(M)-r+1-\# S(M),
$$

where $r \geq 2$. Both contradict Mon3 for $M$. If we are in case ii and a, then

$$
\left|S \cup S_{2}\right|<\left|S_{2}\right|-1+\#\left(S \cup S_{2}\right)(M)-\# S_{2}(M)+1 .
$$

If we are in case ii and $\mathrm{b}$, then

$$
\left|S \cup S_{2}\right|-1<\left|S_{2}\right|-1+\#\left(S \cup S_{2}\right)(M)-r+1-\# S_{2}(M)+1 .
$$

Both of these contradict Mon3 for $M$ as well. We conclude that Mon3 holds for $M^{\prime}$. Hence, normalization preserves the property of being a Mondrian tableau. Observe that the side-length of a square cannot shrink to zero when running the process for Mondrian tableaux.

3.1.4. The algorithm. We now describe a procedure that turns a given Mondrian tableau into a collection of Mondrian tableaux associated to Schubert varieties. We will refer to this algorithm as the Grassmannian Algorithm. We first need a definition.

Definition 3.10. Let $S=\left\{e_{i}, \ldots, e_{j}\right\}$ be a square in a normalized Mondrian tableau $M$. Then a square $N$ of $M$ is a neighbor of $S$ if the following conditions hold:

(1) $e_{j+1} \in N$.

(2) $S$ is not contained in $N$.

(3) If the lower-left corner $l\left(S^{\prime}\right)$ of another square $S^{\prime}$ is between $l(S)$ and $l(N)$, then either $S^{\prime} \subset S$ or $N \subset S^{\prime}$.

Observe that the set of neighbors of a square is totally ordered. Figure 6 shows an example of a square and its neighbors. 


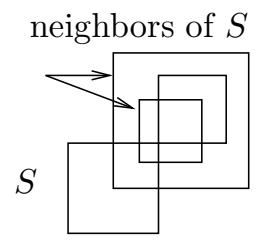

FiguRE 6 . The neighbors of a square.

Definition 3.11 (Distinguished square). Let $M$ be a normalized Mondrian tableau. Among the squares of $M$ that are not nested, the square $S$ whose lower-left corner is furthest southwest is called the distinguished square of $M$.

We can now state the Grassmannian Algorithm. Suppose $M$ is a normalized Mondrian tableau. If $M$ is not the tableau associated to a Schubert variety, we will slide the distinguished square $S$ of $M$ northeast by one unit. This move corresponds to a specialization of the vector spaces defining the variety $\Sigma_{M}$ associated to $M$. As a result, $\Sigma_{M}$ will specialize to a (generically reduced) union of one or more subvarieties each of which is associated to a Mondrian tableau. The algorithm will replace $M$ by the Mondrian tableaux corresponding to the limit subvarieties. We will explain how to determine the limits geometrically in Section 3.2.

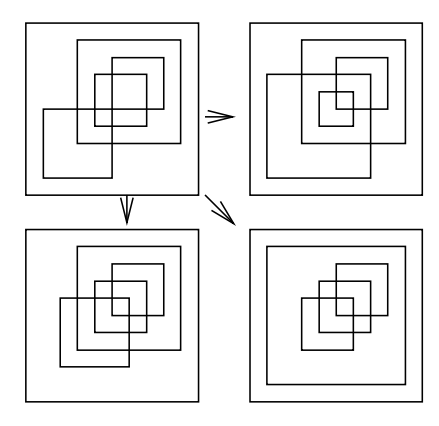

FiguRE 7. Step 2 of the Grassmannian Algorithm.

Algorithm 3.12 (Grassmannian Algorithm). Let $M$ be a Mondrian tableau for $G(k, n)$.

GA1. If $M$ is nested, the algorithm terminates. Otherwise, normalize $M$.

GA2. Let $S$ be the distinguished square of $M$. Let $S^{\prime}$ be the shift of $S$ one unit to the northeast. (If $S=\left\{e_{i}, \ldots,{ }_{j}\right\}$, then $S^{\prime}=\left\{e_{i+1}, \ldots, e_{j+1}\right\}$.) Let $N_{1}, \ldots, N_{r}$ be the set of neighbors of $S$ ordered by their side-lengths in increasing order. For each neighbor $N_{u}$ of $S$, let $M_{u}$ be the tableau obtained from $M$ by replacing $N_{u}$ and $S$ with the intersection $S^{\prime} \cap N_{u}$ and the span $S \cup N_{u}$. Let $M_{0}$ be the tableau obtained from $M$ by replacing $S$ with $S^{\prime}$

If $S^{\prime}$ contains the smallest neighbor $N_{1}$ of $S$, replace $M$ by the tableau $M_{1}$ only. Otherwise, for every neighbor $N_{u}$ of $S$ construct the tableau $M_{u}$. If $S^{\prime}$ is contained in the largest neighbor of $S$ or if $M_{0}$ is not a Mondrian tableau, replace $M$ by the set of tableaux $M_{1}, \ldots, M_{r}$. Otherwise, replace $M$ by the set of tableaux $M_{0}, M_{1}, \ldots, M_{r}$. See Figure 7 for an example.

GA3. Return to Step 1 and run the algorithm for each of the tableaux $M_{i}$ constructed in Step 2.

Remark 3.13. GA2 should be interpreted as asserting the following statement. Let $M$ be a Mondrian tableau. If $S^{\prime}$ contains the smallest neighbor of $S$, then $\left[\Sigma_{M}\right]=\left[\Sigma_{M_{1}}\right]$. Suppose $S^{\prime}$ does not contain the smallest neighbor of $S$. If $S^{\prime}$ is contained in the largest neighbor of $S$ or if $M_{0}$ is not a Mondrian tableau, then $\left[\Sigma_{M}\right]=\sum_{i=1}^{r}\left[\Sigma_{M_{i}}\right]$. Otherwise, $\left[\Sigma_{M}\right]=\sum_{i=0}^{r}\left[\Sigma_{M_{i}}\right]$.

Remark 3.14. We remark that if $S^{\prime}$ is not contained in $N_{r}$ and $S^{\prime}$ does not contain $N_{1}$, then $M_{0}$ fails to be a Mondrian tableau if and only if $\left|S \cup N_{r}\right|=\left|N_{r}\right|+\#\left(S \cup N_{r}\right)(M)-\# N_{r}(M)$. 
Lemma 3.15. The Grassmannian Algorithm replaces a Mondrian tableau for $G(k, n)$ with Mondrian tableaux for $G(k, n)$.

Proof. By Lemma 3.9 normalizing a Mondrian tableau gives a Mondrian tableau. It is clear that every $M_{i}$ replacing $M$ has the same number of squares as $M$. They clearly satisfy Mon1. $M_{0}$ is by assumption a Mondrian tableau. Hence, in that case there is nothing further to check. We can assume that $i \geq 1$. The collection of squares $M_{1}, \ldots, M_{r}$ are normalized. $M$ and $M_{i}$ differ only in squares $S, N_{i}, S^{\prime} \cap N_{i}$ and $\overline{S N}_{i}$, the span of $S$ and $N_{i}$. Since $l(S)=l\left(\overline{S N}_{i}\right)$ and $l\left(S^{\prime} \cap N_{i}\right)=l\left(N_{i}\right)$, the lower-left corners of the squares in $M_{i}$ are distinct. If $S^{\prime}$ does not contain $N_{1}$, then any two squares that share an upper-right corner in $M_{i}$ also share an upper-right corner in $M$. Hence Condition 2 in Definition 3.6 is also clear. If $S^{\prime}$ contains $N^{\prime}$, then Condition 2 in Definition 3.6 holds for $M_{1}$ because by the definition of the distinguished square every square whose lower-left corner is southwest of $l\left(\overline{S N}_{i}\right)$ contains $l\left(\overline{S N}_{i}\right)$. It follows that Mon2 and Mon3 are satisfied for $M_{i}$ by Remark 3.7 .

The Grassmannian Algorithm begins with a Mondrian tableau and terminates with a collection of tableaux associated to Schubert varieties. Note that the step GA2 replaces a normalized Mondrian tableau with tableaux that satisfy Condition 2 in Definition 3.6. Furthermore, except for possibly Tableau $M_{0}$, the tableaux $M_{i}$ constructed in GA2 are automatically normalized. In $M_{0}$ the lower-left corner of at least one square moves strictly northeast. In $M_{i}$, for $i>0$, the total side-length of the squares increases by one. Since the tableau is contained in an $n \times n$ grid, the algorithm has to terminate.

Remark 3.16. The reason that Condition 2 in Definition 3.8 does not simply read that the squares all have distinct upper-right corners is to avoid infinite loops in the Grassmannian Algorithm. Otherwise, if the distinguished square contains its smallest neighbor, shrinking $\overline{S N}_{1}$ gives back the tableau we started with.

Definition 3.17. A degeneration path for a Mondrian tableau $M$ is a sequence of Mondrian tableaux

$$
M^{1} \rightarrow M^{2} \rightarrow \cdots \rightarrow M^{p}
$$

such that $M^{1}=M, M^{p}$ is the Mondrian tableau associated to a Schubert variety and for every $1 \leq i \leq p-1$ the tableau $M^{i+1}$ is one of the tableaux replacing $M^{i}$ in GA2 in Algorithm 3.12.

The main theorem for Grassmannians is:

Theorem 3.18. Let $M$ be a Mondrian tableau for $G(k, n)$ and let $\Sigma_{M}$ denote the associated subvariety of $G(k, n)$. Express the Poincaré dual of the class of $\Sigma_{M}$ as a sum of Schubert cycles $\sum c_{\lambda} \sigma_{\lambda}$. Then the coefficient $c_{\lambda}$ is equal to the number of degeneration paths starting with $M$ and ending in a Mondrian tableau associated to $\sigma_{\lambda}$.

Theorem 3.18 in particular applies to the intersection of Schubert varieties. More generally the projection of the intersection of two Schubert varieties in any partial flag variety is a variety associated to a Mondrian tableau.

3.1.5. The Littlewood-Richardson rule. We now describe how to multiply two Schubert cycles $\sigma_{\lambda}$ and $\sigma_{\mu}$ in $G(k, n)$.

Algorithm 3.19 (Littlewood-Richardson Rule). $\quad$ LR1 Take the Mondrian tableau associated to $\sigma_{\lambda}$ where all the squares have the same lower-left corner $e_{1}$. Label the squares of this Mondrian tableau $A_{1} \subset A_{2} \subset$ $\ldots \subset A_{k}$. Take the Mondrian tableau associated to $\sigma_{\mu}$ where all the squares have the same upper-right corner $e_{n}$. Label the squares of this Mondrian tableau $B_{1} \subset B_{2} \subset \cdots \subset B_{k}$. Place the two Mondrian tableaux on the same $n \times n$ grid.

LR2 For every $1 \leq i \leq k$ form the square $A B_{i}=A_{i} \cap B_{k-i+1}$. If any of the squares $A B_{i}$ is empty, stop. The product of the two Schubert cycles is zero. Otherwise form the Mondrian tableau $M(\lambda, \mu)$ consisting of the squares $A B_{1}, \ldots, A B_{k}$.

LR3 Run the Grassmannian Algorithm on the Mondrian tableau $M(\lambda, \mu)$. 
Remark 3.20. Provided that the intersection of two Schubert varieties is non-empty, then the initial tableau corresponding to the intersection is normalized. This is clear from the construction.

A corollary of Theorem 3.18 is the following Littlewood-Richardson rule.

Theorem 3.21 (Littlewood-Richardson rule). The Littlewood-Richardson coefficient $c_{\lambda, \mu}^{\nu}$ for $G(k, n)$ is equal to the number of degeneration paths starting with $M(\lambda, \mu)$ in an $n \times n$ grid and ending in a Mondrian tableau associated to $\sigma_{\nu}$ in Algorithm 3.19 .

We illustrate Theorem 3.21 by calculating $\sigma_{2,1}^{2}$ in $G(3,6)$.

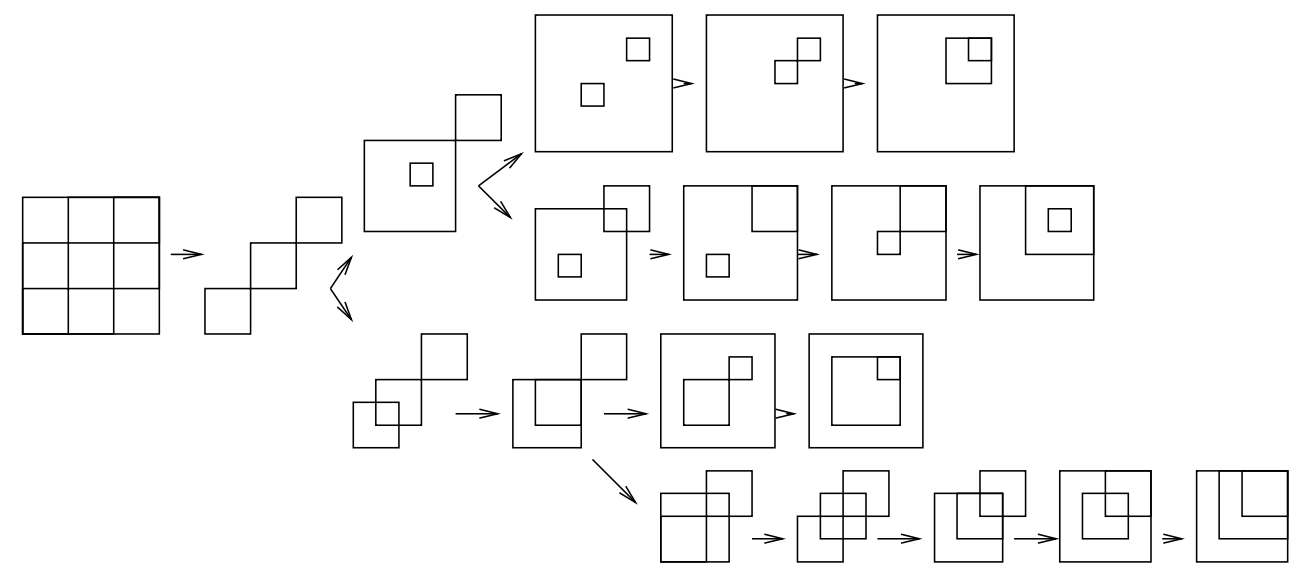

Figure 8 . The product $\sigma_{2,1}^{2}=\sigma_{3,3}+2 \sigma_{3,2,1}+\sigma_{2,2,2}$ in $G(3,6)$.

3.1.6. A more efficient algorithm. The efficiency of the Littlewood-Richardson rule given in Theorem 3.21 can be improved by eliminating unnecessary steps in the Grassmannian Algorithm. In this subsection we state a variation of the Littlewood-Richardson rule. This modified algorithm is important for characterizing the minimal set of Mondrian tableaux that are needed to calculate the Littlewood-Richardson coefficients for all $G(k, n)$.

Definition 3.22. A Littlewood-Richardson tableau is a Mondrian tableau consisting of squares $D_{1}, \cdots, D_{s}$ and $A B_{s+1}, \ldots, A B_{k}$ such that

(1) Either the collection of squares $D_{1} \subset D_{2} \subset \cdots \subset D_{s}$ is nested; or there is a unique square $D_{t}$ such that the collection of $D$ squares omitting $D_{t}$ is nested, $D_{t} \subset D_{u}$ for $u>t$ and the lower-left and upper-right corners of $D_{t}$ are strictly northeast of the lower-left and upper-right corners of $D_{u}$ for $u<t$.

(2) For every $s+1 \leq i \leq k$, the lower-left and upper-right corners of $A B_{h}$ are strictly northeast of the lower-left and upper right corners of $A B_{h-1}$ and of all the squares $D_{u}$. If two squares $D_{u} \subset D_{v}$ have the same lower-left corners, then the $D$ squares contained in $D_{v}$ are nested.

(3) $D_{t-1}$ touches or intersects $D_{t}$. $D_{s}$ touches or intersects $A B_{s+1}$. AB $B_{i}$ touches or intersects $A B_{i+1}$ for every $s+1 \leq i \leq k-1$.

Algorithm 3.23 (Simplifying Littlewood-Richardson Tableaux). Let $M$ be a Littlewood-Richardson tableau.

(1) If the tableau is nested, stop. The algorithm terminates. If the $D$ squares are nested, let the active square be $D_{s}$. Otherwise, there is a unique $D$-square $D_{t}$ as in Definition 3.22. Let the active square be $D_{t-1}$. Slide the active square northeast by one unit. If there are any squares that are contained in the active square and share the same lower-left corner, slide those squares northeast by one unit as well. Denote the resulting tableau by $M_{2}$ 
(2) If the active square is $D_{t-1}$, then its only neighbor is $D_{t}$. If the active square is $D_{s}$, then its only neighbor is $A B_{s+1}$. Form the tableau $M_{1}$ by replacing the active square and its neighbor in $M_{2}$ with their new intersection (the intersection in $M_{2}$ ) and the old span (the span in $M$ ) of the active square and its neighbor. If the last condition of the definition is not satisfied for the largest square contained in the active square, slide it northeast until it touches the lower-left corner of the new intersection, dragging any square that becomes lower-left justified with it.

(3) If after the move the active square does not contain its neighbor or is not contained in its neighbor and if $M_{2}$ is a Mondrian tableau, then replace $M$ by the two tableaux $M_{1}$ and $M_{2}$. Otherwise, replace $M$ by $M_{1}$ only. Apply the algorithm to each of the resulting tableaux.

Algorithm 3.24 (The Modified Littlewood-Richardson Rule). MLR1 Run steps LR1 and LR2 of the Algorithm 3.19 to form the Mondrian tableau $M(\lambda, \mu)$. If there are any basis elements that are not contained in any of the squares of $M(\lambda, \mu)$ remove them from the grid.

MLR2 Apply the Algorithm 3.23 to $M(\lambda, \mu)$.

It is clear that after step MLR1, the resulting tableau is a Littlewood-Richardson tableau. It is also clear that the algorithm for simplifying Littlewood-Richardson tableaux transforms each Littlewood-Richardson tableau to one or two Littlewood-Richardson Tableaux and terminates in a collection of tableaux associated to Schubert varieties.

Theorem 3.25 (Littlewood-Richardson Rule II). The Littlewood-Richardson coefficient $c_{\lambda, \mu}^{\nu}$ is the number of times the Mondrian tableau associated to $\sigma_{\nu}$ occurs in the Modified Littlewood-Richardson Algorithm applied to $M(\lambda, \mu)$.

We illustrate Theorem 3.25 by recalculating $\sigma_{2,1}^{2}$ in $G(3,6)$.

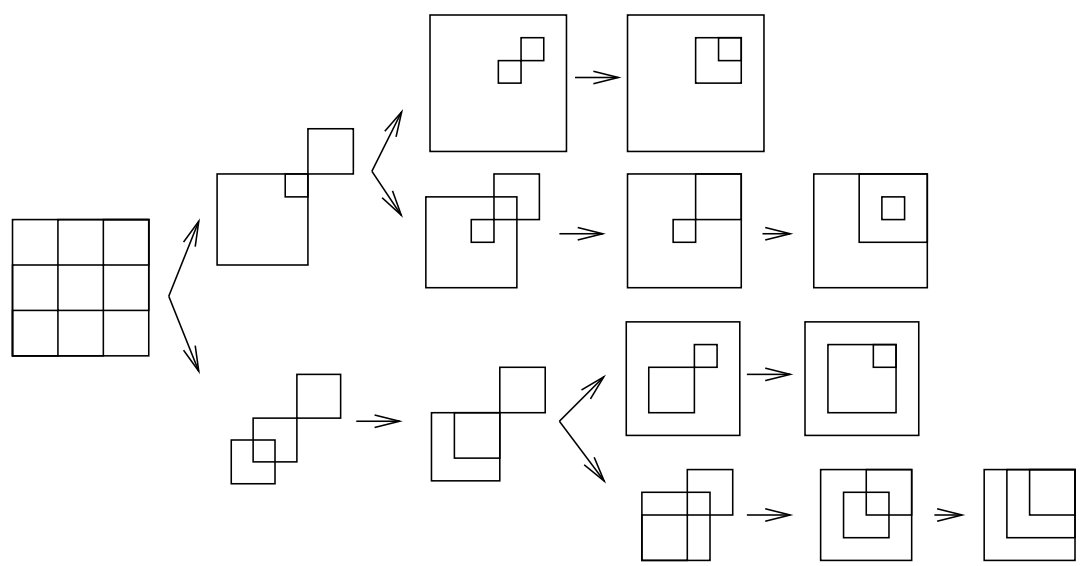

Figure 9. The product $\sigma_{2,1}^{2}=\sigma_{3,3}+2 \sigma_{3,2,1}+\sigma_{2,2,2}$ in $G(3,6)$.

\subsection{The geometry of Mondrian tableaux.}

3.2.1. The variety associated to a Mondrian tableau. In this subsection we explain the geometry behind Mondrian tableaux. We show how to associate a subvariety of the Grassmannian to each Mondrian tableau.

Notation 3.26. For the rest of this paper, we will denote combinatorial objects, such as squares in a Mondrian tableau, by capital letters in the math font (e.g., $S_{i}$ ). We will denote the geometric objects represented by the combinatorial objects by the corresponding letter in Roman font (e.g., $\left.\mathrm{S}_{i}\right)$. 
In a Mondrian tableau, a square of side-length $s$ represents a vector space of dimension $s$. The unit squares along the diagonal of the $n \times n$ grid represent the basis elements $e_{1}, \ldots, e_{n}$. The square represents the vector space spanned by the basis elements it contains. Correspondingly, if a square $S_{1}$ is contained in another square $S_{2}$, then the corresponding linear space $\mathrm{S}_{1}$ is a subspace of the linear space $\mathrm{S}_{2}$.

The squares in a Mondrian tableau represent the vector spaces which impose rank conditions on $k$-planes parameterized by $G(k, n)$. The $k$-planes are required to intersect $\mathrm{S}$ in dimension at least equal to the number of squares (inclusive) contained in $S$. For example, in a Mondrian tableau $S_{1} \subset \cdots \subset S_{k}$ associated to the Schubert cycle $\sigma_{\lambda}$, the $k$-planes are required to intersect $\mathrm{S}_{i}$ in dimension at least $i$.

Now we can define the variety associated to arbitrary Mondrian tableaux. Let $M$ be a Mondrian tableau. Recall that for any square $S$ in the $n \times n$ grid, $|S|$ denotes the side-length of $S$. Denote the number of squares of $M$ contained in $S$ (including $S$ if $S$ is a square of $M$ ) by $\# S(M)$.

Definition 3.27. Let $M$ be a Mondrian tableau for $G(k, n)$. Define the variety $\Sigma_{M}$ associated to $M$ to be the closure of the locus of $k$-planes $[\Lambda] \in G(k, n)$ that satisfy

(1) For any square $S$ of $M, \operatorname{dim}(\Lambda \cap S)=\# S(M)$.

(2) For any two squares $S_{i}, S_{j}$ of $M, \operatorname{dim}\left(\Lambda \cap \mathrm{S}_{i} \cap \mathrm{S}_{j}\right)=\#\left(S_{i} \cap S_{j}\right)(M)$.

In other words, $\Sigma_{M}$ is the closure of the locus of $k$-planes $\Lambda$ where one can choose a basis of $\Lambda$ such that each vector space $\mathrm{S}$ corresponding to a square in $M$ contains \#S(M) basis elements and a basis element is contained in $\mathrm{S}_{i}$ and $\mathrm{S}_{j}$ only if it is contained in $\mathrm{S}_{t}$ for some $S_{t} \subset S_{i} \cap S_{j}$, where $S_{i}, S_{j}$ and $S_{t}$ are squares of $M$.

Let $S_{1} \subset S_{2}$ be two squares of $M$ that share the same lower-left corner $e_{i}$. Suppose that the squares of $M$ contained in $S_{1}$ do not have $e_{i}$ as a lower-left corner. Suppose there does not exist a square strictly containing $S_{1}$ and strictly contained in $S_{2}$. Then the locus of $k$-planes that have a $\# S_{1}(M)$-dimensional subspace in $S_{1}$ and a $\# S_{2}(M)$-dimensional subspace in $S_{2}$ is the same as the locus of $k$-planes that have a $\# S_{1}(M)$-dimensional subspace in $S_{1}$ and an $\left(\# S_{2}(M)-1\right)$-dimensional subspace in $S_{2}-\left\{e_{i}\right\}$. Moreover, the same analysis applies with the lower-left replaced by upper-right. Consequently, the variety associated to a Mondrian tableau and to its normalization are the same variety.

Lemma 3.28. Let $M$ be a Mondrian tableau. Let $M^{\prime}$ be its normalization. Then $\Sigma_{M}=\Sigma_{M^{\prime}}$.

We now calculate the dimension of $\Sigma_{M}$.

Lemma 3.29. Let $M$ be a normalized Mondrian tableau. Then the variety associated to $M$ is an irreducible subvariety of $G(k, n)$ of dimension

$$
\sum_{S \in M}|S|-\sum_{S \in M} \# S(M)
$$

Proof. To see that the variety $\Sigma_{M}$ is irreducible, we inductively construct it. A Mondrian tableau consisting of a single square is projective space $\mathbb{P S}$. Hence it is irreducible and of the dimension claimed in the lemma. Suppose inductively the varieties associated to a Mondrian tableau with $k-1$ squares is irreducible of the claimed dimension. Let $M$ be a Mondrian tableau with $k$ squares $S_{1}, \ldots, S_{k}$. Let $S_{1}$ be the square in $M$ whose lower-left corner is furthest southwest. Let $M^{\prime}$ be the tableau obtained from $M$ by removing $S_{1}$. Then the Zariski-open subset of $\Sigma_{M}$ used in the definition is a non-empty Zariski open set in a projective bundle over a Zariski open subset of $\Sigma_{M^{\prime}}$. Namely, over this open subset consider the $(k-1)$-dimensional subspace contained in the union of $\bigcup_{i=2}^{k} S_{i}$. By assumption each of the $k$-planes parameterized by $\Sigma_{M}$ intersect this subspace in a $(k-1)$-dimensional subspace. Note that we need condition Mon3 for this to be possible. Hence, on this open subset we get a well-defined dominant morphism to $\Sigma_{M^{\prime}}$. The fibers are open subsets in a projective space $\mathbb{P}^{|S|-\# S(M)}$. By induction it follows that $\Sigma_{M}$ is irreducible of the claimed dimension.

Remark 3.30. The reason for insisting on condition Mon3 in the definition of a Mondrian tableau is to make sure that $\Sigma_{M}$ is not empty and the dimension is given by Lemma 3.29. Even if a collection of squares $M$ is not a Mondrian tableau, we can associate the quantity $\sum_{S \in M}|S|-\sum_{S \in M} \# S(M)$ to it. We can still associate a variety to $M$ by asking the $k$-planes to intersect the vector spaces represented by the squares of $M$ in dimension at least equal to the number of squares contained in that square. In this case the quantity gives an upper bound on the dimension. 


\subsubsection{Degenerations. We now would like to discuss the geometry underlying the Grassmannian Algorithm.}

We will obtain the combinatorial Littlewood-Richardson rule by interpreting the move on the Mondrian tableau $M$ as a degeneration of the linear spaces defining the subvariety $\Sigma_{M}$ of $G(k, n)$. We will describe the flat limits of this degeneration and see that the flat limit is a union of the varieties associated to the Mondrian tableaux described in the Grassmannian Algorithm each occurring with multiplicity one. It will follow that the class of $\Sigma_{M}$ is a sum of the classes of the varieties associated to the resulting tableaux. We then repeat the process for each of the resulting tableaux.

Let $M$ be a normalized Mondrian tableau that is not nested. Let $S=\left\{e_{i}, \ldots, e_{j}\right\}$ be the distinguished square. The Grassmannian Algorithm instructs us to move $S$ northeast by one unit. This move represents a one-parameter family of vector spaces $\mathrm{S}(t)$ parameterized by $\mathbb{P}^{1}$, where $\mathrm{S}(t)$ is the vector space

$$
\mathrm{S}(t)=<t e_{i}+(1-t) e_{j+1}, e_{i+1}, \ldots, e_{j}>
$$

Corresponding to this one-parameter family of vector spaces, there is a flat one-parameter family of subvarieties of $G(k, n)$, where the general member is isomorphic to $\Sigma_{M}$. The variety $\Sigma_{M}(t)$ is defined in the same way as $\Sigma_{M}$, except that in every occurence the basis element $e_{i}$ is replaced by the vector $t e_{i}+(1-t) e_{j+1}$. Note that $\Sigma_{M}(1)=\Sigma_{M}$. In a Zariski open subset of $\mathbb{P}^{1}$, these varieties are related by a linear transformation given by a change of basis. Hence, they are isomorphic. Consequently, they form a flat family. Let $\Sigma_{M}(0)$ denote the flat limit of this family. Our task at hand is to describe the irreducible components of $\Sigma_{M}(0)$ that have the same dimension as $\Sigma_{M}$ and the multiplicity of $\Sigma_{M}(0)$ along each of these components.

Observation 3.31 (The main geometric observation). For a square $U$, let $U$ denote the vector space spanned by the basis elements contained in $U$. For $t \neq 0$, let $\mathrm{U}(t)$ denote the vector space spanned by the basis elements contained in $U$ unless $U$ contains $e_{i}$ but not $e_{j+1}$. In the latter case, let $\mathrm{U}(t)$ denote the vector space spanned by $t e_{i}+(1-t) e_{j+1}$ and the basis elements contained in $U-\left\{e_{i}\right\}$. Let $S_{1}, \ldots, S_{r}$ be squares of a Mondrian tableau $M$. For $t \neq 0$, let $\mathrm{S}_{1, \ldots, r}(t)$ denote the span of the vector spaces $\mathrm{S}_{1}(t), \ldots, \mathrm{S}_{r}(t)$. Let $\mathrm{S}_{1, \ldots, r}(0)$ be the flat limit of the spans over $t=0$. Note that the limit of the spans does not have to be the span of the limits. If one of the squares is equal to $S$ and one of the squares contains the basis element $e_{j+1}$ and not $e_{i}$, then the limiting vector space $\mathrm{S}_{1, \ldots, r}(0)$ is the span of $e_{i}$ and the limiting vector spaces $\mathrm{S}_{1}(0), \ldots, \mathrm{S}_{r}(0)$. Otherwise, $\mathrm{S}_{1, \ldots, r}(0)$ is the span of the limiting vector spaces $\mathrm{S}_{1}(0), \ldots, \mathrm{S}_{r}(0)$. Let $S_{1, \ldots, r}(0)$ and $S_{1, \ldots, r}(1)$ denote the squares corresponding to the vector spaces $\mathrm{S}_{1, \ldots, r}(0)$ and $\mathrm{S}_{1, \ldots, r}(1)$, respectively.

The $k$-planes parameterized by $\Sigma_{M}(t)$ intersect the span $\mathrm{S}_{1, \ldots, r}(t)$ in dimension greater than or equal to the number of squares of $M$ contained in $S_{1, \ldots, r}(1)$. Since the condition of intersecting a linear space in at least a given dimension is a closed condition, the $k$-planes parameterized by the flat limit $\Sigma_{M}(0)$ must satisfy the property that they must intersect $\mathrm{S}_{1, \ldots, r}(0)$ in dimension greater than or equal to the number of squares of $M$ contained in $S_{1, \ldots, r}(1)$. Furthermore, this must hold for any set of squares $S_{1}, \ldots S_{r}$ of $M$. We will shortly see that this observation suffices to determine the support of $\Sigma_{M}(0)$.

Every subvariety of the Grassmannian is contained in a variety associated to a Mondrian tableau since for instance the Grassmannian is associated to a Mondrian tableau. In terms of Mondrian tableaux Observation 3.31 asserts that if we are looking for minimal dimensional varieties associated to Mondrian tableaux that support $\Sigma_{M}(0)$ we can restrict our attention to very special tableaux. For any collection of squares $S_{1}(1), \ldots, S_{r}(1)$ in $M$ denote by $S_{1, \ldots, r}(1)$ the union of the basis elements contained in all the squares. Denote by $S_{1, \ldots, r}(0)$ :

- The union of the basis elements in the squares $S_{1}(0), \ldots, S_{r}(0)$ after the distinguished square has been moved if none of the squares contain $e_{i}$ or none of the squares contain $e_{j+1}$.

- The union of $e_{i}$ and the basis elements in $S_{1}(0), \ldots, S_{r}(0)$ if at least one square $S_{j}(1)$ contains $e_{i}$ and one square $S_{j^{\prime}}(1)$ contains $e_{j+1}$.

Then Observation 3.31 asserts that we can assume that a variety associated to a Mondrian tableau $\tilde{M}$ that contains a component of the limit $\Sigma_{M}(0)$ satisfies the property that $\# S_{1, \ldots, r}(0)(\tilde{M}) \geq \# S_{1, \ldots, r}(1)(M)$. We will see that the varieties associated to such Mondrian tableaux have dimension at most the dimension of $\Sigma_{M}$ and those that have the same dimension are the ones described by the Grassmannian Algorithm. Furthermore, they each occur with multiplicity one in the limit. 
Theorem 3.32 (Geometric Formulation of the Algorithm). The support of the flat limit $\Sigma_{M}(0)$ is equal to the union of the varieties associated to the Mondrian tableaux described in Step 2 of the Grassmannian Algorithm. $\Sigma_{M}(0)$ is generically reduced along each of these components.

\subsubsection{The proof that Theorem 3.32 implies Theorems 3.18 and 3.21.}

Proof of Theorem 3.18. We have already observed that the Grassmannian Algorithm transforms a Mondrian tableau to a set of Mondrian tableaux. Furthermore, if one continues applying the algorithm to each of the resulting tableaux, eventually every tableau decomposes to the union of tableaux corresponding to Schubert varieties. We interpret Step 2 of the algorithm as the degeneration described above. Theorem 3.32 implies that the support of the flat limit is the union of the varieties associated to the tableaux described in Step 2 of the algorithm. Moreover, the limit is reduced at the generic point of each of these components. Hence the class of $\Sigma_{M}$ is the sum of the classes of the varieties associated to these tableaux. Theorem 3.18 follows.

Proof of Theorem 3.21. In order to conclude the proof of Theorem 3.21, it suffices to show that the class of the variety $\Sigma_{\lambda} \cap \Sigma_{\mu}$ is equal to the class of the variety associated to the Mondrian tableau described in Step LR2 of Algorithm 3.19.

Initially the two Schubert varieties are defined with respect to two transverse flags. $G L(n)$ acts with a dense orbit on the product of two complete flag varieties. For every pair of flags $\left(F_{\bullet}, G_{\bullet}\right)$ in the dense orbit there is a basis $e_{1}, \ldots, e_{n}$ of the underlying vector space such that $F_{i}=<e_{1}, \ldots, e_{i}>$ and $G_{i}=<e_{n}, e_{n-1}, \ldots, e_{n-i+1}>$. The initial tableau depicts the two Schubert varieties defined in terms of such a pair of opposite flags. Hence, the intersection (if non-empty) is irreducible of dimension $k(n-k)-\sum_{i=1}^{k} \lambda_{i}-\sum_{i=1}^{k} \mu_{i}$. It is easy to see that the intersection is empty if and only if in LR2 one of the squares $A B_{i}$ is empty. Recall that $A B_{i}$ is the intersection of $A_{i}$ and $B_{k-i+1}$. $A_{i}$ (respectively, $B_{k-i+1}$ ) contains an $i$ (respectively, $k-i+1$ ) dimensional subspace of the $k$-planes. Since these two subspaces in a $k$-plane have to intersect, there has to be common basis elements to $A_{i}$ and $B_{k-i+1}$ for every $1 \leq i \leq k$. Conversely, if the squares $A B_{i}$ are non-empty for every $i$, then the span of the basis elements that form the lower-left corner of $A B_{i}$ give a $k$-plane in the intersection. We can now assume that $\Sigma_{\lambda} \cap \Sigma_{\mu}$ is non-empty.

To conclude the proof it suffices to show that the variety associated to $M(\lambda, \mu)$ has the same dimension as $\Sigma_{\lambda} \cap \Sigma_{\mu}$ and has an open subset contained in $\Sigma_{\lambda} \cap \Sigma_{\mu}$. The dimension of the variety associated to $M(\lambda, \mu)$ is by Lemma 3.29 equal to

$$
\sum_{i=1}^{k}\left(n-k+1-\lambda_{i}-\mu_{k-i+1}\right)-k=k(n-k)-\sum_{i=1}^{k} \lambda_{i}-\sum_{i=1}^{k} \mu_{i} .
$$

It follows that the two varieties have the same dimension. Consider the open subset of $M(\lambda, \mu)$ consisting of $k$ planes that have a basis $v_{1}, \ldots, v_{k}$, where $v_{i} \in A B_{i}$ and $v_{i} \notin A B_{j}$ for $j \neq i$. Clearly this open subset is contained in $\Sigma_{\lambda} \cap \Sigma_{\mu}$. Since the two varieties are irreducible of the same dimension and share a dense open subset, we conclude that the two coincide. Hence by Theorem 3.18 Algorithm 3.19 computes the Littlewood-Richardson coefficients.

Proof of Theorem 3.32. The proof of Theorem 3.32 has two steps. In Step 1 we will use the Observation 3.31 to determine the set theoretic components of the flat limit $\Sigma_{M}(0)$. This step will show that the irreducible components of $\Sigma_{M}(0)$ are supported along subvarieties of $G(k, n)$ associated to the Mondrian tableaux described in the Grassmannian Algorithm. Once we know that the set theoretic limits are that simple, in Step 2 an easy local calculation shows that they all occur with multiplicity one.

Observe that if the square $S$ we move starts containing its smallest neighbor $N_{1}$, then the variety associated to the tableau $M_{1}$ is the same variety as the variety associated to $M$. This is simply the reverse of the normalization process for the upper-right corners of the squares. This case should be interpreted not as a degeneration, but as replacing $M$ with the equivalent tableau $M_{1}$ (where two tableaux are equivalent if their associated varieties are equal). In this case there is nothing further to prove. For the rest of the proof we will assume that after moving $S$, its smallest neighbor is not contained in $S$.

Lemma 3.33. The varieties associated to the tableaux $M_{0}, \ldots, M_{r}$ described in Step 2 of the Grassmannian Algorithm have the same dimension as the variety associated to $M$. 
Proof of Lemma 3.33. $M_{0}$ is obtained from $M$ by simply sliding a square one unit. Since the square does not contain its smallest neighbor and is not contained in its largest neighbor, the side-lengths and the containment relations among the squares remain the same. As long as $M_{0}$ is a Mondrian tableau, then the dimension of the associated variety is calculated by Lemma 3.29 and is clearly equal to the dimension of $\Sigma_{M}$.

Let $N_{i}$ be a neighbor of $S$. In the tableau $M_{i}$ the two squares $S$ and $N_{i}$ are replaced by the intersection $S(0) \cap N_{i}$ and the span of $S$ and $N_{i}$. All the other squares remain unchanged. Given two vector spaces the sum of their dimensions is equal to the sum of the dimensions of their intersection and span. Since $\operatorname{dim}\left(\mathrm{S}(0) \cap \mathrm{N}_{i}\right)=\operatorname{dim}\left(\mathrm{S} \cap \mathrm{N}_{i}\right)+1$. We conclude that the total of the side-lengths of the squares in $M_{i}$ is one larger than that of $M$.

On the other hand, the total number of containment relations among squares increases by one as well. Let $T$ be any square of $M$ other than $S$ or $N_{i}$. Let $\# * T(M)$ denote the number of squares of $M$ strictly containing $T$. Then $\# T(M)=\# T\left(M_{i}\right)$ and $\# * T(M)=\# * T\left(M_{i}\right)$.

Since $S$ does not contain the smallest neighbor of $S, S(0) \cap N_{i}$ cannot contain any squares of $M_{i}$ other than $S(0) \cap N_{i}$ that are not already contained in $S$ and $N_{i}$. Hence if any square is contained in both $S$ and $N_{i}$, it is contained in $S(0) \cap N_{i}$ and in the span of $S$ and $N_{i}$. If a square is contained in only one of $S$ or $N_{i}$, then it is contained in their span, but not in $S(0) \cap N_{i}$, unless the square is $S(0) \cap N_{i}$. Finally if a square $T$ is neither contained in $S$ nor in $N_{i}$, it cannot be contained in either of $S(0) \cap N_{i}$ or the span of $S$ and $N_{i}$. A priori $T$ could be contained in the span of $S$ and $N_{i}$. But then the lower-left hand corner would be between $S$ and $N_{i}$. Hence by the definition of a neighbor, $T$ would have to be contained in $S$.

Similarly, any square that contains $S$ necessarily contains $S(0) \cap N_{i}$. If the square also contains $N_{i}$, then it contains their span. If it only contains one of $S$ and $N_{i}$, but not both, then it contains the intersection $S(0) \cap N_{i}$, but not the span of $S$ and $N_{i}$. If it contains both $S$ and $N_{i}$, then it contains both $S(0) \cap N_{i}$ and the span of $S$ and $N_{i}$. Since none of the other squares change, it follows that $\# T(M)=\# T\left(M_{i}\right)$.

Finally to conclude the proof of the lemma, we observe that neither $S$ contains $N_{i}$ nor $N_{i}$ contains $S$. However, the span of $S$ and $N_{i}$ contains $S(0) \cap N_{i}$. By Lemma 3.29 it follows that $\Sigma_{M_{i}}$ has the same dimension as $\Sigma_{M}$.

We can now continue with the proof of Theorem 3.32. Let $Y$ be an irreducible component of the flat limit $\Sigma_{M}(0)$ of dimension equal to the dimension of $\Sigma_{M}$. By the Observation 3.31 the $k$-planes parameterized by $Y$ have to intersect the vector spaces $\mathrm{S}_{i}(0)$ in dimension at least equal to the number of squares in $M$ contained in $S_{i}$. Let $\Lambda$ be a general $k$-plane parameterized by $Y$. Suppose that the $\operatorname{dimension} \operatorname{dim}\left(\Lambda \cap \mathrm{S}_{i}(0)\right)=\# S_{i}(M)$ for every square of $M$ and $\operatorname{dim}\left(\Lambda \cap \mathrm{S}_{i}(0) \cap \mathrm{S}_{j}(0)\right)=\#\left(S_{i} \cap S_{j}\right)(M)$ for every pair of squares $S_{i}$ and $S_{j}$ in $M$. It follows that the support of $Y$ has to be contained in $\Sigma_{M_{0}}$. Since they are both irreducible varieties of the same dimension, we conclude that $\operatorname{supp}(Y)=\Sigma_{M_{0}}$.

Observe $M_{0}$ fails to be a Mondrian tableau when $\left|S \cup N_{r}\right|-\left|N_{r}\right|=\#\left(S \cup N_{r}\right)(M)-\# N_{r}(M)$. In this case, if the limiting positions of the subspaces of the $k$-planes remain independent $\operatorname{dim}\left(\Lambda \cap \mathrm{S}_{i}(0) \cap \mathrm{S}_{j}(0)\right)=\#\left(S_{i} \cap S_{j}\right)\left(M_{0}\right)$, then the dimension of the subspace of $\Lambda$ contained in $N_{r}$ has to increase by one. The same holds if $S(0)$ is contained in $N_{r}$. If there were a component $Y$, by the Observation 3.31 it would be contained in the variety associated to the normalization of $M_{0}$. Note that the variety corresponding to the normalization of $M_{0}$, if nonempty, has dimension strictly smaller than the dimension of $\Sigma_{M}$. It follows that such a locus cannot support an irreducible component of the flat limit. For future reference, we need estimates on this dimension. In this case it is easy to calculate this dimension exactly. Let $e_{r}$ be the lower-left corner of $N_{r}$. Let $p \geq r$ be the largest index such that $e_{r}, e_{r+1}, \ldots, e_{p-1}, e_{p}$ are all lower-left corners of squares in $M$. Suppose $s$ of these basis elements are lower-left corners of squares strictly contained in $N_{r}$. Then the dimension of $\Sigma_{M}$ is $p-r+1-s$ larger than the dimension of the variety associated to the normalization of $M_{0}$ assuming it is non-empty.

We can now assume that the subspaces of $\Lambda$ contained in $\mathrm{S}_{i}(0)$ and $\mathrm{S}_{j}(0)$ for at least two vector spaces $i$ and $j$ intersect in dimension greater than the number of squares contained in $S_{i}(0) \cap S_{j}(0)$. Let T be the smallest dimensional linear space that is the span of consecutive basis elements with the property that the intersection of $\Lambda$ with $\mathrm{T}$ has dimension larger than the number of squares of $M_{0}$ contained in $\mathrm{T}$. If there is more than one vector space with the same dimension, let $\mathrm{T}$ be the one that contains a basis element with least index. List the minimal vector spaces of $M_{0}$ with respect to inclusion $\mathrm{S}_{i_{1}}(0), \ldots, \mathrm{S}_{i_{m}}(0)$ that contain $\mathrm{T}$ in order of their lower-left most corners. The fact that $\Lambda$ intersects $\mathrm{T}$ leads the conditions imposed by $\mathrm{S}_{i_{1}}(0), \ldots, \mathrm{S}_{i_{m}}(0)$ 
to be automatically satisfied. However, by Observation 3.31 any $k$-plane in the flat limit must still intersect the limit of the spans $\mathrm{S}_{i_{h}, i_{h+1}}(0)$ in dimension equal to the number of squares contained in the span of the squares $S_{i_{h}}$ and $S_{i_{h+1}}$ in $M$. Hence, we can replace the tableau $M_{0}$, by the tableau where we delete the squares $S_{i_{1}}(0), \ldots, S_{i_{m}}(0)$ and draw the squares $T, S_{i_{1}, i_{2}}(0), \ldots, S_{i_{m-1}, i_{m}}(0)$. Recall that the squares $S_{i_{h}, i_{h+1}}(0)$ are the spans of the squares $S_{i_{h}}(0)$ and $S_{i_{h+1}}(0)$ unless one of the squares is $S$ and the other square contains $e_{j+1}$ and not $e_{i}$. Call the resulting tableau of squares $U_{1}$. If the dimension of the subspace of $\Lambda$ contained in $\mathrm{S}_{\mathrm{i}}$ for a square $S_{i}$ of $U_{1}$ is equal to $\# S_{i}\left(U_{1}\right)$ and for any two vector spaces $\Lambda \cap \mathrm{S}_{i} \cap \mathrm{S}_{j}=\#\left(S_{i} \cap S_{j}\right)\left(U_{1}\right)$, then by Observation $3.31 Y$ has to be contained in the variety associated to $U_{1}$. Otherwise, we repeat the process. Since this cannot go on indefinitely, we obtain a tableau $U_{a}$ whose associated variety must contain the irreducible component $Y$ of $\Sigma_{M}(0)$.

The first step will be complete if we can show that the variety associated to every tableau other than $M_{1}, \ldots, M_{r}$ obtained by this process has dimension strictly smaller than $\operatorname{dim}\left(\Sigma_{M}\right)$.

First observe that the total side-lengths of the squares in $U_{r}$ is at most one more than the total side-lengths of the squares in $M$. Let $S_{1}, \ldots, S_{j}$ be a collection of squares ordered by their lower-left corners intersecting in a non-empty square. Let $S_{h, h+1}$ denote the span of consecutive squares. Then we have the easy observation that

$$
\sum_{h=1}^{j}\left|S_{h}\right|=\left|S_{1} \cap S_{2} \cap \cdots \cap S_{j}\right|+\sum_{h=1}^{j-1}\left|S_{h, h+1}\right| .
$$

Hence the procedure preserves or decreases the total side-length of the squares of a tableau unless the first square $S_{1}=S(0)$ and $S_{2}$ contains $e_{j+1}$ and not $e_{i}$. (Note that any square with lower-left corner southwest of $S$ contains $S$, so it cannot be minimal if $S$ is in the list.) In the latter case since the side-length of the span is one larger, the side-length increases by one. Since $S$ can occur in the procedure at most once, the total side-length of the squares increases by at most one.

Second we observe that the procedure increases the total number of containment relations by at least $j-1$. Let $R$ be a square other than $S_{1}, \ldots, S_{j}$, then the procedure does not change the number of squares contained in $R$. The procedure also does not change the number of squares containing $R$ unless the lower-left corner of $R$ is between the lower-left corners of $S_{h}$ and $S_{h+1}$ and the upper-right corner of $R$ coincides with the upper-right corner of $S_{h+1}$. If none of the squares are equal to $S$, then this is clear. If $S_{1}=S$, then the argument is identical to the argument in the proof of Lemma 3.33 .

Note that if $j=1$, then the total side-length of the squares has to decrease by at least one since this case corresponds to simply shrinking the side-length of one square. Hence to preserve dimension, we must have $j \geq 2$ for each run of the procedure. However, the total side-length can increase by at most one. We conclude that in order to obtain a variety with dimension equal to $\operatorname{dim}\left(\Sigma_{M}\right)$ we can run the procedure at most once. Furthermore, in this case $j=2, S_{1}=S(0)$ and $e_{j+1} \in S_{2}$. The square $T$ has to be the full intersection of $S(0)$ and $S_{2}$ and if there is a square $S^{\prime}$ whose lower-left corner is between the lower-left corner of $S$ and $S_{2}$ and not contained in $S$, then $S^{\prime}$ must contain $S_{2}$. We conclude that $S_{2}$ has to be a neighbor $N_{i}$ of $S$. Furthermore, the support of the irreducible component $Y$ of $\Sigma_{M}(0)$ has to be contained in $\Sigma_{M_{i}}$. Since both are irreducible of the same dimension we conclude that $\operatorname{supp}(Y)=\Sigma_{M_{i}}$.

We thus conclude that the support of $\Sigma_{M}(0)$ is contained in the union of the varieties associated to the tableaux described by Step 2 of the Grassmannian Algorithm. Furthermore, it is easy to write explicit families of $k$-planes that show that the support of $\Sigma_{M}(0)$ is the union of the varieties associated to the tableaux described by Step 2 of the Grassmannian Algorithm.

There remains to show that $\Sigma_{M}(0)$ is generically reduced along each of these components. It suffices to check the multiplicity at the generic point of each irreducible component. Without loss of generality, we may assume that the Mondrian tableau $M$ consists only of $S$ and the neighbors $N_{1}, \ldots, N_{r}$. Let $\mathcal{M} \rightarrow \mathbb{P}^{1}$ denote the total space of the family of varieties associated to Mondrian tableaux. For $t \neq 0$, let $V(t)$ be the vector space which is the span of $\mathrm{S}(t)$ and $\mathrm{N}_{r}(t)$. Let $V(0)$ denote the limit of $V(t)$. Suppose there are $p$ squares in $M$ contained in the span of the squares $S$ and $N_{r}$. Over a dense Zariski-open subset $\mathcal{U}$ of $\mathcal{M}$ intersecting every component of $\Sigma_{M}(0)$, the morphism obtained by restricting the $k$-planes to their $p$-dimensional subspaces contained in $V(t)$ is a smooth morphism. Let $T$ be a square contained in $S$ or $N_{r}$, but not equal to $S$ or any of the neighbors. After possibly shrinking $\mathcal{U}$ to another Zariski open intersecting every component of $\Sigma_{M}(0)$, the morphism quotienting 
out the $p$-planes by their subspaces contained in $\mathrm{T}$ is a smooth morphism. It follows that to determine the multiplicities it suffices to treat the case when the tableau consists only of $S$ and the neighbors $N_{1}, \ldots, N_{r}$.

By induction this reduces to a computation in the Grassmannian of lines. Suppose $S$ has only one neighbor. Then the multiplicity of each tableau is one and the variety associated to each tableau occurs as a component of $\Sigma_{M}(0)$. This easily follows either by the Pieri rule for the Grassmannian of lines or by an easy direct tangent space calculation. Now suppose $r>1$. For $t \neq 0$, let $V(t)$ denote the span of $\mathrm{S}(t)$ and $\mathrm{N}_{r-1}(t)$. Let $V(0)$ denote the limit of $V(t)$. We can restrict the $(r+1)$-planes to the $r$-plane contained in $V(t)$. By induction it follows that each component occurs with multiplicity one. This concludes the proof of Theorem 3.32.

3.3. Amplifications of Theorem 3.32. In this subsection we discuss some amplifications of Theorem 3.32 . The Grassmannian Algorithm dictates a precise order of degeneration. For many questions, this precise order may be inconvenient. The question arises whether it is necessary to follow this order. In fact, we will shortly observe that the proof of Theorem 3.32 uses very few facts about $S$. We now make this precise.

Let $M$ be a normalized Mondrian tableau. Let $S$ be any square of the Mondrian tableau with the property that any other square $S^{\prime}$ of $M$ with $l\left(S^{\prime}\right)<l(S)$ contains $S$. Call such squares degeneratable squares. Note that the distinguished square of $M$ is such a square.

Let $S$ be a degeneratable square. Then we can slide $S$ northeast by one unit. The new feature in this case is that if there exists any squares that contain $S$ and have the same upper-right corner as $S$, we need to split those squares to two pieces at the lower-left corner of $S$ and slide their parts that coincide with $S$ northeast as well.

If $S$ is the square $S=\left\{e_{i}, \ldots, e_{j}\right\}$, this move corresponds to the degeneration where $e_{i}$ is replaced by $t e_{i}+(1-t) e_{j+1}$. Corresponding to this degeneration of vector spaces, there is a flat family of varieties $\Sigma_{M}(t)$ obtained by applying to $\Sigma_{M}$ the linear transformation for $t \neq 0$ giving the change of basis. Let $\Sigma_{M}(0)$ be the flat limit of this family at $t=0$. We would like to describe $\Sigma_{M}(0)$. In this case the description remains almost identical. For the convenience of the reader we spell this out.

Algorithm 3.34 (A slightly different degeneration). Let $M^{\prime}$ be the tableau where the squares that move are placed in their new position. For any square $T$ of $M$, let $T(1)$ denote the original position of $T$ in $M$ and $T(0)$ denote the new position of $T$ in $M^{\prime}$. Let $N_{1}, \ldots N_{r}$ be the neighbors of $S$ in $M$. Let $M_{0}$ be the normalization of $M^{\prime}$.

If $S(0)$ contains the smallest neighbor of $S$, replace $M$ by the tableau formed by replacing $S(0)$ and $N_{1}$ in $M^{\prime}$ with the span of $S(1)$ and $N_{1}$ and the intersection of $S(0)$ and $N_{1}$. Otherwise, for every neighbor $N_{i}$ form the tableau $M_{i}$ by replacing in $M^{\prime}$ the squares $S(0)$ and $N_{i}$ with the span of $S(1)$ and $N_{i}$ and the intersection of $S(0)$ and $N_{i}$. If $S(0)$ is contained in the largest neighbor $N_{r}$ of $S$ or if $\left|N_{r}\right|+\#\left(S \cup N_{r}\right)(M)-\# N_{r}(M)=\left|S \cup N_{r}\right|$, then replace $M$ by the tableaux $M_{1}, \ldots, M_{r}$. Otherwise, replace $M$ by the tableaux $M_{0}, M_{1}, \ldots, M_{r}$.

Corollary 3.35. The support of the flat limit $\Sigma_{M}(0)$ is equal to the union of the varieties associated to the tableaux described in the Algorithm 3.34. $\Sigma_{M}(0)$ is generically reduced along each of these varieties.

Proof. The statement is an immediate corollary of the proof of Theorem 3.32. If the set theoretic statement is correct, then the fact that $\Sigma_{M}(0)$ is generically reduced along each component follows by the same multiplicity calculation as in the proof of 3.32. The proof that the set theoretic limits are contained in the union of the varieties associated to $M_{0}, M_{1}, \ldots, M_{r}$ is again obtained by a dimension count. We observe that the dimension counts in the proof of Theorem 3.32 only used the fact that any square whose lower-left corner is to the southwest of $l(S)$ contains $S$. Since we are assuming this about $S$, the corollary follows.

The corollary implies that we can obtain many different Littlewood-Richardson Rules by taking different degeneration orders. We also observe that the corollary implies Theorem 3.25.

Proof of Theorem 3.25. Note that the more efficient algorithm eliminates any basis elements that is not used in the description of the variety. This does not change the variety. Similarly the more efficient algorithm slides squares that do not intersect any other squares. We can interpret such slides as a relabeling of the basis elements. Since under this relabeling none of the vector spaces change, the variety associated to the tableau 
does not change. Finally, the more efficient algorithm does not normalize. Note that in Littlewood-Richardson tableaux every square has at most one neighbor. Furthermore, if a square $S_{1}$ shares the same lower-left corner with a square strictly contained in it, then the collection of squares contained in $S_{1}$ is nested. Hence, normalizing preserves the property that there is only one neighbor for Littlewood-Richardson tableaux. Hence by Algorithm 3.34 only Tableau $M_{1}$ can occur if we first normalize and move the squares until we move the active square. Since this is equivalent to moving the active square and sliding the squares contained in it that share the same lower-left corner with it, Theorem 3.25 follows.

Remark 3.36. In addition to being more efficient, the importance of Theorem 3.25 stems from the fact that it singles out Littlewood-Richardson tableaux as the smallest class of tableaux that are necessary to calculate all Littlewood-Richardson coefficients of all Grassmannians by the method proposed in this paper.

Corollary 3.35 indicates that in the definition of Mondrian tableaux one should relax the condition that squares be spans of consecutive basis elements. This also is a useful feature when tackling many problems. In particular, in the algorithm for two-step flag varieties we would like to allow for vector spaces that are not spans of consecutive basis elements.

Definition 3.37. A square $S$ is called chopped if it is not the span of consecutive basis elements. The maximal intervals of the basis elements contained in $S$ are called the chops of $S$. The lower-left most chop lch $(S)$ is the chop that contains $l(S)$. Let $e_{i}$ be a basis element such that $e_{i} \notin S$. Suppose that there exists indices $h<i<j$ such that $e_{h}, e_{j} \in S$. We will then call $e_{i}$ a gap of $S$.

We will depict a chopped square $S$ in a Mondrian tableau by deleting the parts of the square along the row and column corresponding to a gap of $S$.

Allowing arbitrary collections of squares makes using and working with Mondrian tableau fairly difficult and is not necessary for most applications. We will settle on an intermediate ground and allow our squares to be chopped in certain simple ways.

Definition 3.38 (Generalized Mondrian Tableau). A generalized Mondrian tableau $M$ is a collection of (possibly chopped) squares satisfying conditions Mon2 and Mon3 and the following two conditions:

Mon1a No square is contained strictly to the southwest of a gap of another square.

Mon1b Let $S$ and $S^{\prime}$ be any two squares in $M$ with the property that $l\left(S^{\prime}\right) \geq l(S)$. Then either one of the two squares contains the other and if $g$ is a gap of both they coincide northeast of $g$. Or the gaps of $S^{\prime}$ are a subset of the gaps of $S, r\left(S^{\prime}\right) \geq r(S)$ and if $g$ is a gap of $S^{\prime}, S$ and $S^{\prime}$ coincide northeast of $g$.

Let $M$ be a collection of squares satisfying Mon1a and Mon1b. If $S$ and $S^{\prime}$ in $M$ are two squares with $l(S)=l\left(S^{\prime}\right)$, then either $S \subset S^{\prime}$ or $S^{\prime} \subset S$. Let $g$ be the first gap northeast of $l(S)$. Say it is a gap of $S$. By definition either $S \subset S^{\prime}$ or $S$ and $S^{\prime}$ agree northeast of $g$. Since $S$ and $S^{\prime}$ agree southwest of $g$ as well, we still have $S \subset S^{\prime}$. The definition of a normalized tableau has to be slightly revised to account for the fact that the squares are no longer spans of consecutive basis elements.

Definition 3.39. We say that a generalized Mondrian tableau $M$ is normalized if

(1) The lower-left corners of any two squares of $M$ are distinct.

(2) Let $S \subset S^{\prime}$ be any two squares of $M$. If the upper-right corner of $l c h(S)$ equals the upper-right corner of lch $\left(S^{\prime}\right)$, then every square whose lower-left corner is southwest of $l\left(S^{\prime}\right)$ contains $S$.

We will always work with tableaux that satisfy Condition 2 in Definition 3.39. We note that a slight modification of Algorithm 3.8 transforms a generalized Mondrian tableau satisfying Condition 2 in Definition 3.39 to a generalized Mondrian tableau satisfying Condition 1.

Algorithm 3.40 (Normalizing generalized Mondrian tableaux.). Let $M$ be a collection of squares satisfying Mon1a and Mon1b. Suppose there are squares that share the same lower-left corner. Let $e_{i}$ be the southwest most basis element which is the lower-left corner of more than one square. List $S_{1} \subseteq \cdots \subseteq S_{r}$ the squares that have $e_{i}$ as their lower-left corner. Remove $e_{i}$ from $S_{2}$ and label the resulting square $S_{2}^{\prime}$. Replace $S_{2}$ in $M$ with $S_{2}^{\prime}$ and label the resulting collection $M^{\prime}$. If all the lower-left corners of the squares in $M^{\prime}$ are distinct, stop. 
Otherwise, repeat by replacing $M$ with $M^{\prime}$. Discard the tableau if the side-length of any square shrinks to zero.

After finitely many applications $M$ will be a collection of squares with distinct lower-left corners. If $M$ is normalized, stop. Otherwise there is a basis element $e_{i}$ which is the upper-right corner of $l \operatorname{ch}(S)$ and $l \operatorname{ch}\left(S^{\prime}\right)$ for a pair of squares $S \subset S^{\prime}$ and not all squares $S^{\prime \prime}$ with $l\left(S^{\prime \prime}\right)<l\left(S^{\prime}\right)$ contain $S$. Let $e_{i}$ be the northeast most basis element with this property. Let $S_{1} \subseteq S_{2} \subseteq \cdots \subseteq S_{r}$ be the set of squares containing $e_{i}$ as the upper-right corner of their lower-left chops and that fail Condition 2 in Definition 3.39. Remove $e_{i}$ from $S_{2}$ and label the resulting square $S_{2}^{\prime}$. Let $M^{\prime}$ be the collection of squares obtained by replacing $S_{2}$ in $M$ by $S_{2}^{\prime}$. If $M^{\prime}$ is normalized, stop. Otherwise, repeat by replacing $M$ by $M^{\prime}$. If the side-length of any of the squares shrink to zero, discard the tableau.

Lemma 3.41. Let $M$ be a collection of squares satisfying Mon1a and Mon1b. Applying the Algorithm 3.40 to the lower-left hand corners of the squares in M preserves Mon1a and Mon1b.

Proof. Mon1a clearly holds when we shrink lower-left corners: If $S_{2}^{\prime}$ is contained southwest of a gap of a square $S$ when we shrink $l\left(S_{2}\right)$, then $S_{2}$ is also contained southwest of $S$. This contradicts Mon1a for $M$. Mon1b holds when we shrink lower-left corners. We only need to check it for $S_{2}^{\prime}$. Suppose $l(S)<l\left(S_{2}\right)$. If $S_{2} \subset S$ in $M$, then $S_{2}^{\prime} \subset S$ in $M^{\prime}$. If $g$ is a gap of $S_{2}^{\prime}$ and $S$ in $M^{\prime}$, then $g$ is a gap of $S_{2}$ and $S$ in $M$, hence $S_{2}^{\prime}$ and $S$ agree northeast of $g$. Otherwise, $r(S) \leq r\left(S_{2}\right)$ and any gap of $S_{2}$ is a gap of $S$ and $S_{2}$ and $S$ agree northeast of any gap $g$ of $S_{2}$ by Mon1b for $M$. Since $r\left(S_{2}\right)=r\left(S_{2}^{\prime}\right)$ and the gaps of $S_{2}^{\prime}$ are a subset of the gaps of $S_{2}$, Mon1b holds for these squares. If $l(S)=l\left(S_{2}\right)$, either $S_{2} \subset S$ and Mon1b holds as before. Else $S=S_{1}$. If $g$ is a gap of $S_{2}^{\prime}$, then $g$ must also be a gap of $S_{1}$, hence the two agree northeast of $g$ and Mon1b holds. If $l(S)>l\left(S_{2}\right)$ and $S \subset S_{2}$ in $M$, then $S \subset S_{2}^{\prime}$ in $M^{\prime}$. If $g$ is a gap of $S$ and $S_{2}^{\prime}$ in $M^{\prime}$, then $g$ is a gap of $S$ and $S_{2}$ in $M$. Since $S$ and $S_{2}$ agree in $M$ northeast of $g, S_{2}^{\prime}$ and $S$ agree northeast of $g$ in $M^{\prime}$. If $l(S)>l\left(S_{2}\right)$ and $S \not \subset S_{2}$ in $M$, then if $l\left(S_{2}^{\prime}\right) \leq l(S)$ and $g$ is a gap of $S_{2}^{\prime}$, then $g$ is a gap of $S_{2}$ in $M$, hence Mon1b holds. If $l\left(S_{2}^{\prime}\right)<l(S)$, since the gaps of $S$ are a subset of the gaps of $S_{2}$ and $r(S) \geq r\left(S_{2}\right)=r\left(S_{2}^{\prime}\right)$, we must have $S_{2}^{\prime} \subset S$. If $g$ is a gap of $S_{2}^{\prime}$ and $S$ in $M^{\prime}$, then $g$ is a gap of $S_{2}$ and $S$ in $M$, hence $S_{2}^{\prime}$ and $S$ agree northeast of $g$.

The final amplification of Theorem 3.32 we will discuss is an algorithm for finding the classes of varieties associated to generalized Mondrian tableaux. Define the variety $\Sigma_{M}$ associated to a generalized Mondrian tableau $M$ as in Definition 3.27. The proof of Lemma 3.29 holds without change. Hence $\Sigma_{M}$ is an irreducible subvariety of $G(k, n)$ of dimension $\sum_{S \in M}|S|-\sum_{S \in M} \# S(M)$.

Algorithm 3.42. Let $S$ be a square of a normalized, generalized Mondrian tableau $M$ with the property that any square whose lower-left corner is southwest of $l(S)$ contains $S$. Move the $l \operatorname{ch}(S)$ northeast by one unit. If any square $S^{\prime}$ containing $S$ has a chop whose upper-right corner coincides with the upper-right corner of $l \operatorname{ch}(S)$, chop $S^{\prime}$ at $l(S)$ and slide the chop of $S^{\prime}$ coinciding with $l \operatorname{ch}(S)$ by one unit as well. Suppose $l \operatorname{ch}(S)$ is given by $\left\{e_{i}, \ldots, e_{j}\right\}$, then define a neighbor of $S$ exactly as in Definition 3.10. Apply Algorithm 3.34.

Lemma 3.43. Algorithm 3.42 transforms a normalized, generalized Mondrian tableau to normalized, generalized Mondrian tableaux of the same dimension.

Proof. For a square $T$, let $T(0)$ denote the new position of $T$ in $M^{\prime}$. Let $T(1)$ denote the old position of $T$ in $M$. The tableaux $M_{1}, \ldots, M_{r}$ are normalized. The lower-left corners of all the squares are clearly distinct since they are the same as the lower-left hand corners of the squares in $M$. The upper-right hand corners of the lower-left chops of the squares are also as in $M$, except possibly if $S(0)$ contains its smallest neighbor $N_{1}$. If $S(0)$ or a square containing $S(0)$ contains $N_{1}$, then every square whose lower-left corner is southwest of $S$ also contains $N_{1}$. Condition 2 in Definition 3.39 holds for any other square since it holds for $M$. By an argument analogous to that in Remark 3.7, $M_{1}, \ldots M_{r}$ satisfy Mon2 and Mon3.

In $M_{i}$ let $I=S(0) \cap N_{i}$ and $U=S \cup N_{i}$. If $U$ or any of the squares $T(0)$ that move are contained southwest of a gap $g$ of another square, then $S$ and $T$ would be contained southwest of $g$ in $M$ contradicting Mon1a for $M$. Suppose $I$ were contained entirely to the southwest of a gap of a square $X(0)$. If $l(X(0))<l(S)$, then $S \subset X(1)$ in $M$. Hence, $g$ is a gap of $S(0)$. Either $g$ is also a gap of $N_{i}$, in which case $N_{i}$ and $S$ agree northeast of $g$ contradicting that $I$ is contained southwest of $g$. Otherwise $g$ is not a gap of $N_{i}$, hence $r(S(0)) \in N_{i}$ by Mon1b for $M$. This contradicts that $I$ is contained southwest of $g$. If $l(X)>l(S)$, then either $X \subset S$ or $S$ 
agrees with $X$ northeast of $g$. If $X \subset S$ and $g$ is a gap of $S$, then $X$ and $S$ agree northeast of $g$. Otherwise, $g \in I$. By Mon1b for M, the gaps of $N_{i}$ are a subset of the gaps of $S$. Hence in any of the cases $I$ cannot be contained southwest of $g$. We conclude that Mon1a holds for $M_{i}$.

If $X$ and $Y$ are squares of $M_{i}$ neither of which are in the set $\{U, I\}$, then Mon1b holds for $X$ and $Y$. If $l(X)<l(S)$, then $I \subset X$ since $S(0) \subset X(0)$. Suppose $g$ is a gap of $I$ and $X$. Then $g$ is a gap of $S(0)$. Hence $S(0)$ and $X$ agree northeast of $g$. If $g$ is also a gap of $N_{i}$, then $X, S$ and $N_{i}$, hence also $I$ agree northeast of $g$. If $g$ is a gap of $U$, then it is a gap of both $N_{i}$ and $S$, hence either $X$ contains $N_{i}$ and $S$ or agrees with both northeast of $g$. Therefore, either $X$ contains $U$ or agrees with $U$ northeast of $g$. If $X$ does not contain $N_{i}$, then the gaps of $N_{i}$ are a subset of the gaps of $X$. Note that the gaps of $U$ are a subset of the gaps of $N_{i}$. Hence the gaps of $U$ are a subset of the gaps of $X$. If $l(S)<l(X)<l(N)$, then either $X \subset S$ or $N \subset X$. If $N \subset X$, then a gap of $X$ is also a gap of $N$ and consequently also a gap of $S$. $S$ and $N$ agree northeast of $g$ and they agree with $X$. Hence both $U$ and $I$ agree with $X$ northeast of $g$. Also $g$ is a gap of both $U$ and $I$. If $X \subset S$, then $X \subset U$. If $g$ is a gap of both $X$ and $U$, then $g$ is a gap of $S$ and $N$, hence they all agree northeast of $g$. If $g$ is a gap of $I$, then it is a gap of $S(0)$ and hence of $X$. Since $S(0)$ and $X$ agree northeast of it, $X$ and $I$ agree northeast of it as well. If $l(X)>l\left(N_{i}\right)$, either $N_{i}$ contains $X$ or the gaps of $X$ are a subset of the gaps of $N_{i}$ and hence also of $S$. In the latter case the gaps of $X$ are clearly a subset of the gaps of $U$ and $I$. If $g$ is a gap of $X$ both $U$ and $I$ agree with it northeast of $g$. If $N_{i}$ contains $X$, then $U$ contains $X$. If $g$ is a gap of both $U$ and $X$, then $S$ and $N_{i}$ agree with $X$ northeast of $g$, hence so does $U$. If $I$ contains $X$, then the argument is the same as in the previous case. If $I$ does not contain $X$, then $S$ does not contain $X$. The gaps of $X$ are contained in the gaps of $S$ and hence of $I$. If $g$ is a gap of $X$, then $S(0)$ agrees with $X$ northeast of $g$ and so does $I$. We conclude that Mon1b holds for the Tableau $M_{i}$.

Finally, whenever $M_{0}$ occurs, $M^{\prime}$ satisfies Mon1a and Mon1b and Condition 2 in Definition 3.39. If $r(l \operatorname{ch}(S(0)))=r\left(l \operatorname{ch}\left(S^{\prime}\right)\right)$ in $M^{\prime}$, then $r(l \operatorname{ch}(S(1)))=r\left(l \operatorname{ch}\left(S^{\prime}(1)\right)\right)$ in $M$. Otherwise, $S(0)$ would contain its smallest neighbor, hence $M_{0}$ would not occur. We might need to normalize the lower-left corner of $S(0)$. By Lemma 3.41 normalizing the lower-left hand corners preserves Mon1a and Mon1b. In this case it is easy to see that Condition 2 in Definition 3.39 is preserved. We conclude that $M_{0}$ is a normalized, generalized Mondrian tableau. Finally, the proof of Lemma 3.33 remains unaltered. This concludes the proof of the lemma.

We interpret the move described in Algorithm 3.42 as the usual degeneration of the variety $\Sigma_{M}$. Let $\Sigma_{M}(0)$ denote the flat limit of this family. The fact that Algorithm 3.42 can be used to compute the classes of generalized Mondrian tableaux follows from the following corollary of Theorem 3.32.

Corollary 3.44. Let $M$ be a generalized Mondrian tableau. Consider the flat family corresponding to the move of generalized Mondrian tableau. Then the support of the flat limit $\Sigma_{M}(0)$ is equal to the union of the varieties associated to the tableaux described by Algorithm 3.42. $\Sigma_{M}(0)$ is generically reduced along each of those varieties.

Proof. It suffices to show that the supports of the irreducible components of $\Sigma_{M}(0)$ are contained in the union of the claimed varieties. The multiplicity calculations remain unchanged. We use Observation 3.31 to determine the set theoretic limits. We observe that the basic dimension estimate in the proof of Theorem 3.32 remains unchanged for generalized Mondrian tableaux. The proof of Theorem 3.32 depended on the estimate that if in the limit the $k$-planes intersect a linear space contained in the intersection of the limiting positions of $j>1$ minimal linear spaces with respect to inclusion, then the dimension decreases by at least $j-1$ if one of the vector spaces is not $S(0)$ and by at least $j-2$ if one of the vector spaces is $S(0)$. Once we know this the corollary immediately follows as in the proof of Theorem 3.32. By the Observation 3.31 this is easy to see. Preserving the notation in the proof of Theorem 3.32 , suppose $\mathrm{T}$ is minimally contained in $\mathrm{S}_{i_{1}}(0), \ldots, \mathrm{S}_{i_{j}}(0)$. If $T$ intersects the lower-left chops of all the squares, the proof remains unchanged. The sum of the side-lengths of the squares increase by at most one and the containment relations increase by at least $j-1$. If $T$ is not contained in the lower-left chops of all the squares $S_{i_{h}}$, then taking the spans of consecutive squares increases the total side-length by the total number $G$ of gaps $S_{i_{h}}$ has along the lower left chop of $S_{i_{h+1}}$ as $h$ varies from 1 to $j-2$. Note that here we are using that $S_{i_{h}}$ and $S_{i_{h+1}}$ agree northeast of the first gap of $S_{i_{h+1}}$. However, in that case, requiring $\Lambda$ to intersect $\mathrm{S}_{i_{h}, i_{h+1}}(0)$ in dimension $\# S_{i_{h}, i_{h+1}}(M)$ does not account for Observation 3.31. $\Lambda$ has to also intersect $S_{i_{h}, i_{h+2}}(0)$ in dimension $\# S_{i_{h}, i_{h+2}}(M)$. This imposes $G$ conditions on $\Lambda$. It follows that if $j>1$, the dimension decreases by at least $j-1$. This concludes the proof of Corollary 3.44. 


\section{Painted Mondrian tableaux and a Littlewood-Richardson Rule for two-SteP FlaG} VARIETIES.

In this section we obtain a Littlewood-Richardson rule for two-step flag varieties. Recall that $F\left(k_{1}, k_{2} ; n\right)$ denotes the two-step flag variety that parameterizes pairs of vector spaces $V_{1} \subset V_{2}$ of dimensions $k_{1}$ and $k_{2}$, respectively, of a fixed $n$-dimensional vector space $V$. We preserve the notation from $\S 2.1$ and $\S 3$.

4.1. Preliminary combinatorial definitions. In this subsection we introduce combinatorial objects called painted Mondrian tableaux. As in $\S 3.1$, fix a basis $e_{1}, \ldots, e_{n}$. A square will denote a span of the basis elements. In addition, the squares will now have one of two colors: red or black. The black squares will denote the vector spaces that impose rank conditions on $V_{2}$. The red squares will denote the vector spaces that impose rank conditions on $V_{1}$.

Notation 4.1. Let $M$ be a collection of red and black squares. For any square $S$, let $|S|$ denote the side-length of the square $S$. Let $\#_{B} S(M)$ denote the number of black squares (inclusive) of $M$ contained in $S$. Let $\#{ }_{R} S(M)$ denote the number of red squares (inclusive) of $M$ contained in $S$.

Definition 4.2. Let $R$ be a chopped red square in a collection of red and black squares $M$. A gap of $R$ is a unit square $e_{i} \notin R$ such that $l(R)<e_{i}<r(R)$ and $e_{i}$ is a gap of a black square contained in $R$. A hole of $R$ is a unit square $e_{i} \notin R$ such that $l(R)<e_{i}<r(R)$, but $e_{i}$ is not contained in any black squares $B$ with $l(B) \geq l(R)$.

Definition 4.3. A painted Mondrian tableau $M$ for $F\left(k_{1}, k_{2} ; n\right)$ is a collection of distinct black squares $B_{1}, \ldots, B_{k_{2}}$ and distinct red squares $R_{1}, \ldots, R_{k_{1}}$ such that

PM1 The black squares $B_{1}, \ldots, B_{k_{2}}$ form a generalized Mondrian tableau for $G\left(k_{2}, n\right)$.

PM2 Each red square is the span of the black squares it contains.

PM3a No red square is contained southwest of a gap of another red square. If $g$ is a gap of a square $S$ of $M$, then there exists a black square $B \subset S$ with $l(B)>g$. If $e_{i}$ is a basis element not contained in a red square $R$ and $l(R)<e_{i}<r(R)$, then $e_{i}$ is either a gap or a hole of $R$.

PM3b Let $R$ and $R^{\prime}$ be two red squares of $M$ with $l\left(R^{\prime}\right) \geq l(R)$. Either one of the squares is contained in the other and if $g$ is a gap of both $R$ and $R^{\prime}$, then their gaps coincide northeast of $g$. Or $r\left(R^{\prime}\right) \geq r(R)$ and if $g$ is a gap of $R^{\prime}$, then either $g$ is a gap of $R$ or there does not exist a black square $B$ with $l(B) \geq l\left(R^{\prime}\right)$ contained in $R$ and containing $g$. Furthermore, in the latter case if $g$ is a gap of both $R$ and $R^{\prime}$, then the gaps of $R$ and $R^{\prime}$ coincide northeast of $g$.

PM3c Let $g$ be a gap of a red square $R$. If $S$ and $S^{\prime}$ are two black squares of $M$ contained in $R$ with $l\left(S^{\prime}\right)<$ $g<l(S)$, then $S \subset S^{\prime}$.

PM4 No red square is the span of the red squares it strictly contains.

PM5 Let $R_{i}$ and $R_{j}$ be any two red squares in $M$. Then

$$
\left|R_{i} \cup R_{j}\right| \geq\left|R_{i}\right|+\#_{R}\left(R_{i} \cup R_{j}\right)(M)-\#_{R} R_{i}(M) ;
$$

and

$$
\#_{B}\left(R_{i} \cup R_{j}\right)(M) \geq \#_{B} R_{i}(M)+\#_{R}\left(R_{i} \cup R_{j}\right)(M)-\#_{R} R_{i}(M)
$$

We will place the basis elements $e_{1}, \ldots, e_{n}$ on an $n \times n$ grid ordered from southwest to northeast. We will depict the red squares in red and the black squares in black. In order to not clutter the diagrams if a red square coincides with a black square, we will only draw the red square. This should not cause any confusion. Figure 10 depicts some examples.

A painted Mondrian tableau $M$ represents an irreducible subvariety $\Sigma_{M}$ of the two-step flag variety. We will describe how to associate a subvariety to a painted Mondrian tableau in detail in Section 4.3. Roughly, $\Sigma_{M}$ is the closure of the locus of pairs $\left(V_{1}, V_{2}\right) \in F\left(k_{1}, k_{2} ; n\right)$ where $V_{1}$ (respectively, $\left.V_{2}\right)$ intersects the subspaces spanned by the basis elements contained in red (respectively, black) squares in specified dimensions. Denote by $\left[\Sigma_{M}\right]$ the Poincaré dual of the class of $\Sigma_{M}$.

It is easy to represent Schubert varieties in two-step flag varieties by painted Mondrian tableaux. 


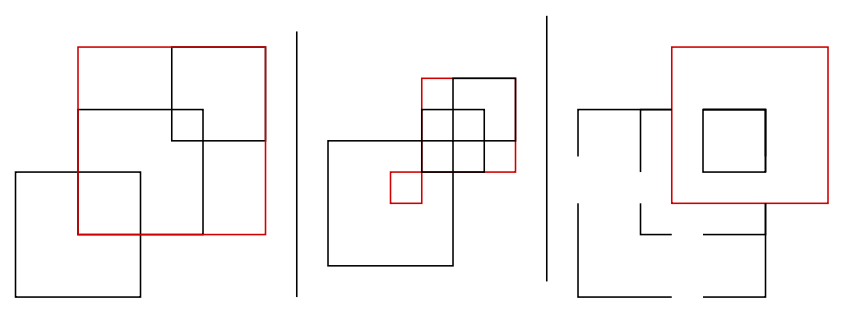

Figure 10. Three examples of painted Mondrian tableaux.

Definition 4.4. The painted Mondrian tableau $M(\lambda, \delta)$ associated to the Schubert cycle $\sigma_{\lambda}^{\delta}$ is a painted Mondrian tableau consisting of $k_{2}$ nested black squares $B_{1} \subset \cdots \subset B_{k_{2}}$, where the side-length of $B_{i}$ is $n-k_{2}+i-\lambda_{i}$, and $k_{1}$ red squares $R_{1} \subset \cdots \subset R_{k_{1}}$ coinciding with the black squares to which $\delta$ assigns the index 1 .

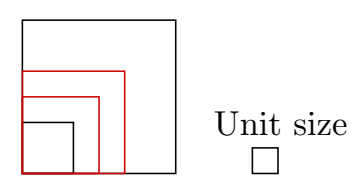

Figure 11. A painted Mondrian tableau associated to $\sigma_{1,1,1,0}^{2,1,1,2}$ in $F(2,4 ; 6)$.

Given the intersection of two Schubert varieties in $F\left(k_{1}, k_{2} ; n\right)$, we can also associate a painted Mondrian tableau to the intersection. We now describe this procedure.

Algorithm 4.5 (The Algorithm for associating a painted Mondrian tableau to a Schubert problem.). Let $\sigma_{\lambda}^{\delta}$ and $\sigma_{\mu}^{\kappa}$ be two Schubert cycles in $F\left(k_{1}, k_{2} ; n\right)$.

1 Let $M(\lambda, \delta)$ be the painted Mondrian tableau associated to $\sigma_{\lambda}^{\delta}$ where the squares are not chopped and all the squares have $e_{1}$ as their lower-left corner. Similarly, let $M(\mu, \kappa)$ be the painted Mondrian tableau associated to $\sigma_{\mu}^{\kappa}$ where the squares are not chopped and all the squares have $e_{n}$ as their upper-right corner. Place $M(\lambda, \delta)$ and $M(\mu, \kappa)$ in the same $n \times n$ grid. Label the black squares of $M(\lambda, \delta)$ by $A_{1} \subset \cdots \subset A_{k_{2}}$ and the red squares by $A R_{1} \subset \cdots \subset A R_{k_{1}}$. Label the black squares of $M(\mu, \kappa)$ by $C_{1} \subset \cdots \subset C_{k_{2}}$ and the red squares by $C R_{1} \subset \cdots \subset C R_{k_{1}}$.

2 For every $1 \leq i \leq k_{2}$, if $r\left(A_{i}\right)<l\left(C_{k_{2}-i}\right)$ shrink the tableau by removing the rows and columns corresponding to the basis elements between $r\left(A_{i}\right)$ and $l\left(C_{k_{2}-i}\right)$. Replace every square with its restriction to this tableau. Keep the labeling of the squares the same.

3 Starting with $i=1$ and proceeding in increasing order apply the following procedure. Consider the smallest remaining black square $A_{i}$ of $M(\lambda, \delta)$. If $A_{i}$ does not coincide with a red square in $M(\lambda, \delta)$, draw the intersection of $A_{i}$ with the largest remaining black square $C_{j}$ in $M(\mu, \kappa)$ in black. Delete both $A_{i}$ and $C_{j}$. If $A_{i}$ coincides with a red square $A R_{j}$ in $M(\lambda, \delta)$ and $C R_{k_{1}-j+1}$ is smaller than the largest remaining black square in $M(\mu, \kappa)$, form the intersection of $A_{i}$ with the black square coinciding with $C R_{k_{1}-j+1}$ in $M(\mu, \kappa)$ in black. Delete both squares. Otherwise form the intersection of $A_{i}$ with the largest remaining black square in $M(\lambda, \delta)$ in black. Delete both squares. Label the resulting square $B_{i}$. If any of the intersections is empty, stop. The algorithm terminates. The intersection of the Schubert varieties is empty.

4 For every $1 \leq i \leq k_{1}$, draw the intersection of the red squares $A R_{i} \cap C R_{k_{1}-i+1}$ in red. Delete $A R_{i}$ and $C R_{k_{1}-i+1}$. Label the intersection $R_{i}$. If any of the $R_{i}$ are empty, stop. The algorithm terminates.

5 Define the Mondrian tableau associated to the intersection $M\left(\sigma_{\lambda}^{\delta}, \sigma_{\mu}^{\kappa}\right)$ to be the painted Mondrian tableau consisting of the black squares $B_{i}$ and red squares $R_{i}$.

Figure 12 gives three examples of Algorithm 4.5.

Definition 4.6. A painted Mondrian tableau is called normalized if the following two conditions hold: 


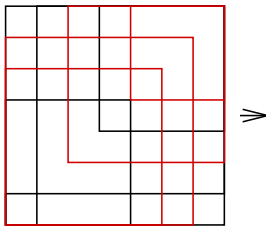

.
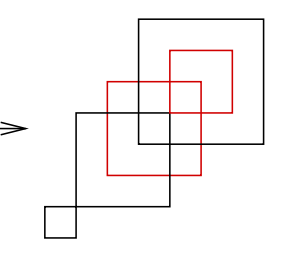
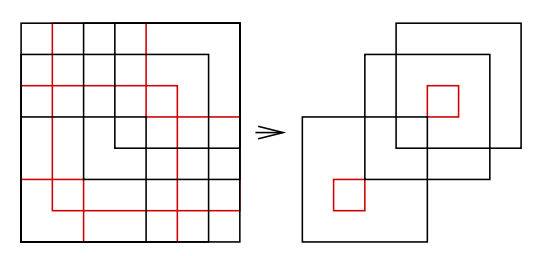

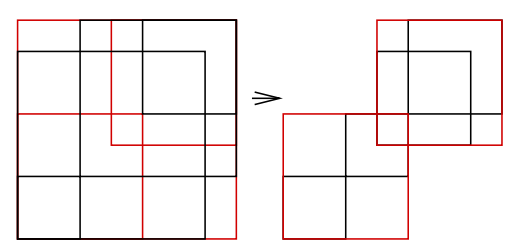

Figure 12. Three examples for Algorithm 4.5.

(1) Any two squares of the tableau that have the same color have distinct lower-left corners.

(2) Let $S \subset S^{\prime}$ be any two squares of the tableau of the same color such that $l \operatorname{ch}(S)$ and $l \operatorname{ch}\left(S^{\prime}\right)$ have the same upper-right corner. Then every square whose lower-left corner is southwest of $l\left(S^{\prime}\right)$ contains $S$.

Remark 4.7. As in Remark 3.7, Conditions PM4 and PM5 automatically hold for a normalized painted Mondrian tableau.

The following definition will play an important role in determining the degeneration order.

Definition 4.8. Let $S$ be a black square of a normalized painted Mondrian tableau $M$. A black square $F$ of $M$ is called a filler in $S$ if $F$ is strictly contained in $S$ and $F$ shares the same lower-left corner with a red square not contained in $S$.

It is possible to describe limits of degenerations for varieties associated to painted Mondrian tableaux. However, for obtaining a Littlewood-Richardson rule we do not need to work with such general tableaux. We will make further assumptions on the painted Mondrian tableaux to simplify the geometry and the algorithm.

Definition 4.9. A normalized painted Mondrian tableau $M$ for $F\left(k_{1}, k_{2} ; n\right)$ is called admissible if the following additional conditions hold.

APM1 If there is a containment relation between two distinct red squares $R_{1} \subset R_{2}$ of $M$, then any square $S$ of $M$ with $l(S) \leq l\left(R_{2}\right)$ contains $R_{1}$.

APM2 If there is a containment relation between two distinct black squares $B_{1} \subset B_{2}$ of $M$, then either any square $S$ of $M$ with $l(S) \leq l\left(B_{2}\right)$ contains $B_{1}$ or $B_{1}$ is contained in a red square $R$ of $M$ strictly contained in $B_{2}$. If $B_{1}$ does not coincide with $R$, then any square $S$ with $l(S) \leq B_{2}$ either contains $B_{1}$ or contains a filler $F$ with $l(F)<l\left(B_{1}\right)$.

APM3 Let $R_{1} \subset B$ be a red square strictly contained in a black square of $M$. Let $B_{1} \subset R_{1}$ be a square that does not coincide with $R$ northeast of $l\left(B_{1}\right)$. Then either $B_{1}$ is contained in every square $S$ with $l(S) \leq l(B)$ or there exists a red square $R_{2} \subset B$ strictly contained in $B$ with $l\left(R_{2}\right)<l\left(R_{1}\right)$ and $B_{1} \subset R_{2}$.

Definition 4.10. Let $M$ be a normalized painted Mondrian tableau for $F\left(k_{1}, k_{2} ; n\right)$. Let $S$ be a square of any color in $M$. A red neighbor of $S$ is a neighbor of $S$ in the tableau consisting of $S$ and the red squares of $M$. A black neighbor of $S$ is a neighbor of $S$ in the tableau consisting of $S$ and the black squares of $M$.

Lemma 4.11. The tableau associated to the intersection of two Schubert varieties (if non-empty) is normalized and admissible.

Proof. We can assume that the intersection of any of the squares is non-empty. The following are clear from the construction:

(1) The black squares are not chopped since they are intersections of non-chopped squares. Hence Mon1a and Mon1b vacuously hold.

(2) No two black squares share an upper-right or lower-left corner since the lower-left (respectively, upperright) corners of the squares correspond to distinct lower-left (respectively, upper-right) corners of the tableau associated to $\sigma_{\mu}^{\kappa}$ (respectively, $\sigma_{\lambda}^{\delta}$ ). The latter are all distinct. Similarly, the lower-left and upper-right corners of red squares are distinct. Hence PM1, PM4 and PM5 hold. 
(3) The red squares are not chopped. They are intersections of non-chopped red squares. Furthermore, every square contained in a red square is contained in a black square contained in it. The red squares are the spans of the black squares contained in them. The reason for Step 2 in Algorithm 4.5 is to ensure these last two statements hold. Otherwise, the red squares could have holes. We would then have to restrict the red squares so that they are the spans of black squares contained in them. In any case, PM2, PM3a,b,c hold.

(4) There are no containment relations among any two red squares. There may be containment relations among black squares. However, in the construction a square of the tableau associated to $\sigma_{\lambda}^{\delta}$ does not intersect the largest remaining black square, only when it coincides with a red square. In that case the black square formed coincides with the corresponding red square. Hence APM1, APM2 and APM3 hold.

From these observations it is clear that the tableau corresponding to the intersection of two Schubert varieties is a normalized, admissible painted Mondrian tableau.

Definition 4.12. Let $M$ be a painted Mondrian tableau. Define the dimension of a tableau $M$ to be

$$
\sum_{i=1}^{k_{2}}\left|B_{i}\right|+\sum_{i=1}^{k_{1}} \#_{B} R_{i}(M)-\sum_{i=1}^{k_{2}} \#_{B} B_{i}(M)-\sum_{i=1}^{k_{1}} \#_{R} R_{i}(M)
$$

Lemma 4.13. The tableau associated to a Schubert variety $\Sigma_{\lambda}^{\delta}$ where $\delta$ assigns 1 to the indices $i_{1}<\cdots<i_{k_{1}}$ has dimension

$$
\sum_{l=1}^{k_{2}}\left(n-k_{2}-\lambda_{l}\right)+\sum_{j=1}^{k_{1}}\left(i_{j}-j\right)
$$

equal to the dimension of the Schubert variety.

Proof. This is immediate from the definition of the dimension of a tableau.

Lemma 4.14. Assuming it is non-empty, the tableau associated to the intersection of two Schubert varieties $\Sigma_{\lambda}^{\delta}$ and $\Sigma_{\mu}^{\kappa}$ where $\delta$ associates 1 to the indices $i_{1}<\cdots<i_{k_{1}}$ and $\kappa$ associates 1 to the indices $j_{1}<\cdots<j_{k_{1}}$ has dimension

$$
\sum_{l=1}^{k_{2}}\left(n-k_{2}-\lambda_{l}-\mu_{k_{2}-l+1}\right)+\sum_{l=1}^{k_{1}}\left(i_{l}+j_{k_{1}-l+1}-k_{2}\right)-k_{1},
$$

equal to the dimension of intersection of the Schubert varieties $\Sigma_{\lambda}^{\delta}$ and $\Sigma_{\mu}^{\kappa}$.

Proof. The sum of the side-lengths of the black squares is $\sum_{l=1}^{k_{2}}\left(n-k_{2}-\lambda_{l}-\mu_{k_{2}-l+1}+1\right)$. If $k_{2}-i_{l}+1 \leq j_{k_{1}-l+1}$, then $R_{l}$ contains $j_{k_{1}-l+1}-k_{2}+i_{l}$ black squares. If $k_{2}-i_{l}+1>j_{k_{1}-l+1}$, then $k_{2}-i_{l}+1-j_{k_{1}-l+1}$ black squares strictly contain the black square $B_{i_{l}}$ and $R_{l}$ contains one black square. Hence, the dimension of the tableau is equal to

$$
\sum_{l=1}^{k_{2}}\left(n-k_{2}-\lambda_{l}-\mu_{k_{2}-l+1}+1\right)+\sum_{l=1}^{k_{1}}\left(-k_{2}+i_{l}+j_{k_{1}-l+1}\right)-k_{1}-k_{2} .
$$

This is equal to dimension claimed in the lemma. Note that the dimension of the intersection of the Schubert varieties (if non-empty) is equal to this as well.

4.2. The combinatorial Littlewood-Richardson rule. We now state the Littlewood-Richardson rule for two-step flag varieties. The algorithm for computing the classes of painted Mondrian tableau will be very similar to the Grassmannian Algorithm. We will move squares in the tableau and replace the tableau by new tableaux. The move on the Mondrian tableau $M$ corresponds to a degeneration of the vector spaces defining the variety $\Sigma_{M}$. In the flat limit the variety $\Sigma_{M}$ will specialize to the union of one or more varieties associated to painted Mondrian tableaux. The algorithm replaces the original tableau with the tableaux corresponding to the limiting varieties. As in the case of the Grassmannians, the limits are determined by the requirement that the limiting vector spaces have to intersect the limits of the vector spaces spanned by the basis elements contained in the squares of $M$ and the spans of these vector spaces in given dimensions. We now make this discussion precise. 
Rule 4.15 (The rule for locating the square to move). Let $M$ be a normalized, admissible painted Mondrian tableau. If $M$ is nested, there is no square to move. The algorithm terminates. Otherwise, among the squares of $M$ that are not nested, let $T_{1}$ be the black square whose lower-left corner is furthest southwest. For $i>1$, set $T_{i}$ be the black square contained in $T_{i-1}$ whose lower-left corner is furthest southwest. Among the squares $T_{i}$, let $T_{h}$ be the square with smallest index satisfying the following two properties:

(1) $T_{h}$ does not contain any fillers.

(2) The collection of squares contained in $T_{h}$ is nested.

Set $S=T_{h}$ to be the square to move.

Rule 4.16 (The rule for moving squares). Let $M$ be a normalized, admissible painted Mondrian tableau. Let $S$ be the square determined by Rule 4.15. We move $S$ by shifting the lower-left most chop of $S$ northeast by one unit. If there are any squares (red or black) $S^{\prime}$ that contain $S$ and have a chop whose upper-right corner coincides with the upper-right corner of $l \operatorname{ch}(S)$, we chop $S^{\prime}$ at $l(S)$ and move the chop coinciding with $l c h(S)$ northeast by one unit. (Note that in case $l c h(S)=l \operatorname{ch}\left(S^{\prime}\right)$ this means we move $l \operatorname{ch}\left(S^{\prime}\right)$ as well.) We keep all other squares fixed.

After moving $S$ according to Rule 4.16, we will replace $M$ by a collection of tableaux. As usual, we need a procedure for normalizing the resulting tableaux.

Algorithm 4.17 (Normalizing painted Mondrian tableaux.). Let $M$ be a collection of red and black squares where the black squares satisfy Mon1a and Mon1b, the red squares satisfy PM2, PM3a, PM3b. If $M$ is normalized, stop. If the tableaux consisting of black squares is not normalized, run Algorithm 3.40 on the black squares of $M$. If the resulting tableau is normalized, stop. Otherwise, if two red squares share a common lower-left corner, let $e_{i}$ be the southwest most unit square that is the lower-left corner of two red squares. Let $S_{1} \subset \cdots \subset S_{r}$ be all the red squares that have $e_{i}$ as their lower-left corner. Consider the black squares contained in $S_{2}$ whose lower-left corners are strictly northeast of the lower-left corner of $S_{2}$. Let $B$ be the square whose lower-left corner is southwest most among them. Shrink $S_{2}$ by moving its lower-left corner northeast to coincide with the lower-left corner of $B$. Repeat until no two red squares share a common lower-left corner. If during the procedure the side-length of a square shrinks to zero, discard the tableau. If the tableau is not normalized repeat the procedure for the upper-right corners of the red squares failing Condition 2 in Definition 4.6 starting with the northeast most basis element.

Let $S(0)$ be the new position of the square $S$ and let $S(1)$ refer to the old position. If $S$ coincides with a red square $R$ in $M$, let $R(1)$ denote the old position of $R$ and $R(0)$ the new position of $R$. Let $M^{\prime}$ denote the tableau where all the squares that move are placed in their new position and all other squares are kept as in $M$.

$M_{0}$ If any of the red squares in $M^{\prime}$ is not equal to the span of the black squares contained in it, shrink the red square so that it is the span of the black squares contained in it. Normalize the resulting tableau. See Figure 13 for examples.

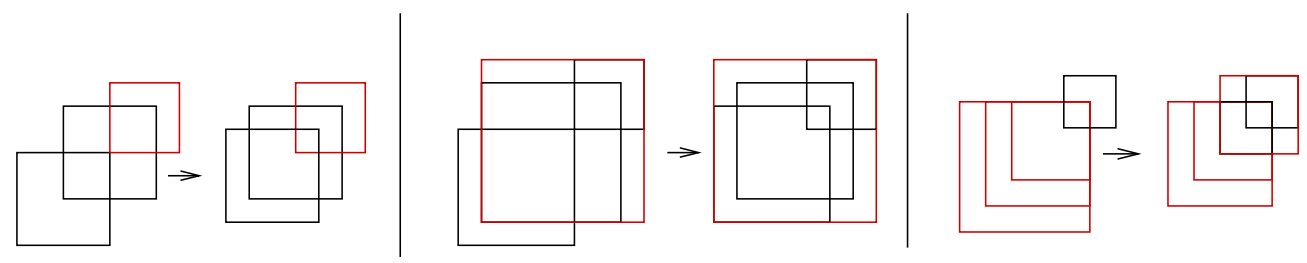

Figure 13. Some examples of Tableau $M_{0}$.

$M_{1}(N R)$ Suppose $S$ coincides with a red square $R$ of $M$. For a red neighbor $N R$ of $R$ form the tableau $M_{1}(N R)$ as follows: Replace the red squares $N R$ and $R(0)$ in $M^{\prime}$ with the intersection $R(0) \cap N R$ and the span of $R(1)$ and $N R$ in red. List all the black squares $S(0)=B_{i_{1}}, \ldots, B_{i_{j}}$ in $M^{\prime}$ minimal with respect to inclusion that contain the intersection $R(0) \cap N R$. Order them by their lower-left corners (southwest 
to northeast). Replace the black squares $B_{i_{1}}, \ldots, B_{i_{j}}$ in $M^{\prime}$ with the following squares drawn in black: $S(0) \cap N R$, the span of $S(1)$ and $B_{i_{2}}$ and the spans of $B_{i_{h}}$ and $B_{i_{h+1}}$ for $2 \leq h \leq j-1$. If any of the red squares is not equal to the span of the black squares contained in it, shrink the red square so that it is the span of the black squares contained in it. Normalize the resulting tableau. Figure 14 gives an example of this tableau.

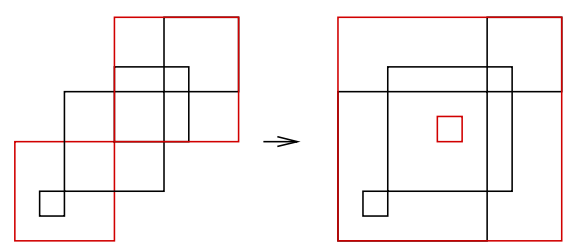

Figure 14. An example of Tableau $M_{1}\left(N R_{i}\right)$.

$M_{2}\left(N_{i}\right)$ For every black neighbor $N_{1}, \ldots, N_{r}$ of $S$ in $M$ form the tableau $M_{2}\left(N_{i}\right)$ as follows: Replace $S(0)$ and $N_{i}$ in $M^{\prime}$ with the intersection $S(0) \cap N_{i}$ and the span of $S(1)$ and $N_{i}$ in black. If any of the red squares $R$ is not the span of the black squares contained in it, shrink $R$ by removing the basis elements from $R$ that are not contained in black squares contained in $R$. Normalize the resulting tableau. See Figure 15 for examples.

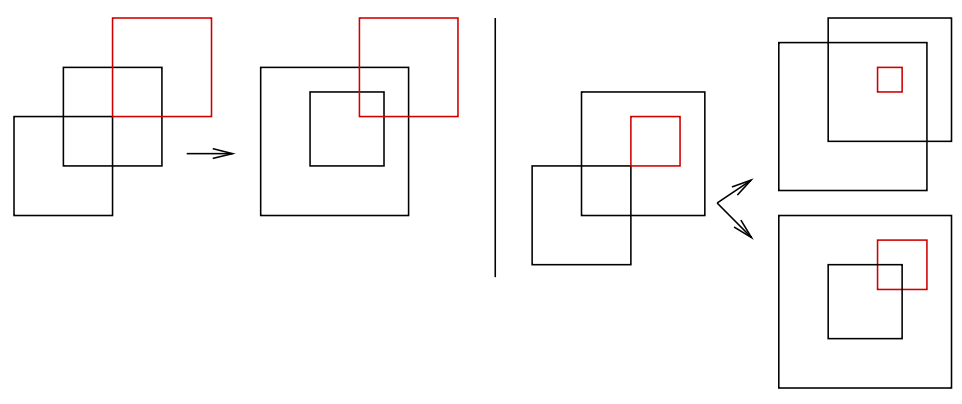

Figure 15. Some examples of Tableau $M_{2}\left(N R_{i}\right)$.

$M_{3}(N R)$ Suppose the largest black neighbor $B_{1}$ of $S$ in $M$ coincides with a red square $R_{1}$. If $B_{h-1}$ in $M$ coincides with a red square $R_{h-1}$, set $B_{h}$ to be the black square with southwest most lower-left corner among the black squares satisfying $l\left(B_{h}\right)>l\left(R_{h-1}\right)$ and $B_{h} \not \subset R_{h-1}$. Among the squares $B_{1}, B_{2}, \ldots$, suppose $B_{l+1}$ is the black square with least index which does not coincide with a red square. Then draw the tableau $M_{3}(N R)$ as follows. Replace $S(0), B_{1}, \ldots, B_{l+1}$ in $M^{\prime}$ with the black squares $S(0) \cap B_{1}, B_{1} \cap$ $B_{2}, \ldots, B_{l} \cap B_{l+1}$ and the span of $S(1), B_{1}, B_{2}, \ldots, B_{l+1}$. If any of the intersections are empty, discard the tableau. If any of the red squares is not equal to the span of the black squares contained in it, shrink the red square so that it is the span of the black squares contained in it. Normalize the resulting tableau. See Figure 16 for examples.

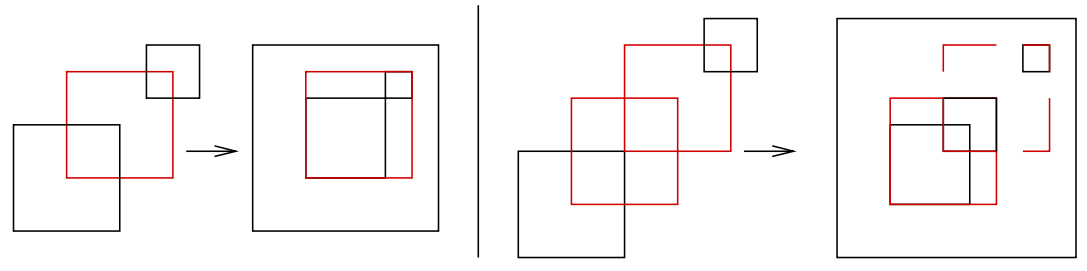

Figure 16. Some examples of Tableau $M_{3}(N R)$. 
Algorithm 4.18 (The algorithm for simplifying painted Mondrian tableaux.). Let $M$ be a normalized, admissible painted Mondrian tableau. If $M$ is nested stop. Otherwise, let $S$ be the square determined by the Rule 4.15 . Move $S$ following Rule 4.16. Replace $M$ by the tableaux among $M_{0}, M_{1}(N R), M_{2}\left(N_{i}\right)$ and $M_{3}(N R)$ that have the same dimension as $M$. Repeat the process for each of the resulting tableaux.

Algorithm 4.19 (Littlewood-Richardson Rule for two-step flag varieties). Given two Schubert cycles $\sigma_{\lambda}$ and $\sigma_{\mu}$ form the painted Mondrian tableau associated to their intersection following Algorithm 4.5. Apply Algorithm 4.18 for simplifying painted Mondrian tableaux until every tableau is the tableau associated to a Schubert variety.

We will see in Proposition 4.41 that each normalized, admissible painted Mondrian tableau is replaced by normalized, admissible painted Mondrian tableaux and that the algorithm terminates. Hence at the end the tableau corresponding to the intersection of two Schubert varieties is replaced by a collection of tableaux associated to Schubert varieties.

Definition 4.20. A degeneration path for a painted Mondrian tableau $M$ is a sequence of painted Mondrian tableaux

$$
M^{1} \rightarrow M^{2} \rightarrow \cdots \rightarrow M^{p}
$$

such that $M^{1}=M, M^{p}$ is the Mondrian tableau associated to a Schubert variety and for every $1 \leq i \leq p-1$ the tableau $M^{i+1}$ is one of the tableaux replacing $M^{i}$ in Algorithm 4.18

The main theorem of this paper is the following theorem which asserts that Algorithm 4.19 is indeed a Littlewood-Richardson rule for two-step flag varieties.

Theorem 4.21 (Littlewood-Richardson Rule for two-step flag varieties). Let $\sigma_{\lambda}$ and $\sigma_{\mu}$ be two Schubert cycles in the two step flag variety $F\left(k_{1}, k_{2} ; n\right)$. Let their product be $\sigma_{\lambda}^{\delta} \cdot \sigma_{\mu}^{\kappa}=\sum_{\nu} c_{\lambda \mu}^{\nu} \sigma_{\nu}^{\alpha}$. The coefficient $c_{\lambda \mu}^{\nu}$ is equal to the number of degeneration paths starting with the tableau $M\left(\sigma_{\lambda}^{\delta}, \sigma_{\mu}^{\kappa}\right)$ in an $n \times n$ grid and ending in a painted Mondrian tableau associated to $\sigma_{\nu}^{\alpha}$.

The proof of Theorem 4.21 is very similar to the proof of Theorem 3.21. We interpret the transformations of the Mondrian tableaux as degenerations of the vector spaces represented by the Mondrian tableaux. There are corresponding flat families of subvarieties of $F\left(k_{1}, k_{2} ; n\right)$. The Algorithm 4.18 records the flat limit of these degenerations. The degenerations eventually terminate in varieties associated to Schubert varieties. In $\S 4.3$ we discuss this more precisely.

Remark 4.22. As in the case of Grassmannians, it is possible to make the Algorithm 4.19 more efficient. This was done in the author's original formulation of the rule. We refer the reader to the author's original manuscript.

4.2.1. Examples. Before discussing the geometry of painted Mondrian tableaux and proving Theorem 4.21, we calculate some intersections in two-step flag varieties using Algorithm 4.19.

We first calculate

$$
\sigma_{1,0,0}^{2,1,2} \cdot \sigma_{1,1,0}^{2,1,2}=\sigma_{2,1,0}^{1,2,2}+\sigma_{2,2,0}^{2,1,2}+\sigma_{1,1,1}^{1,2,2}
$$

in $F(1,3 ; 5)$. See Figure 17 for the calculation.

Next we calculate

$$
\sigma_{1,0,0,0}^{2,1,2,1} \cdot \sigma_{1,0,0,0}^{2,1,2,1}=\sigma_{1,1,0,0}^{1,2,2,1}+\sigma_{1,1,1,0}^{2,1,2,1}+\sigma_{1,1,0,0}^{2,1,1,2}+\sigma_{1,1,1,1}^{2,2,1,1}
$$

in $F(2,4 ; 5)$. See Figure 18 for the calculation.

We now begin discussing some basic properties of admissible, normalized, painted Mondrian tableaux.

Lemma 4.23. Let $M$ be a normalized, painted Mondrian tableau. If a black square $B$ coincides with a red square $R$, then $B$ cannot be a filler in any of the squares that contain it.

Proof. Suppose $B \subset T$ is a filler. Then $l(B)=l\left(R^{\prime}\right)$ for some red square $R^{\prime}$ not contained in $T$. Since $M$ is normalized and $l(B)=l(R), l(B)$ cannot be the lower-left corner of any red squares other than $R$. Since $B$ and $R$ coincide, $R \subset T$ leading to a contradiction. 


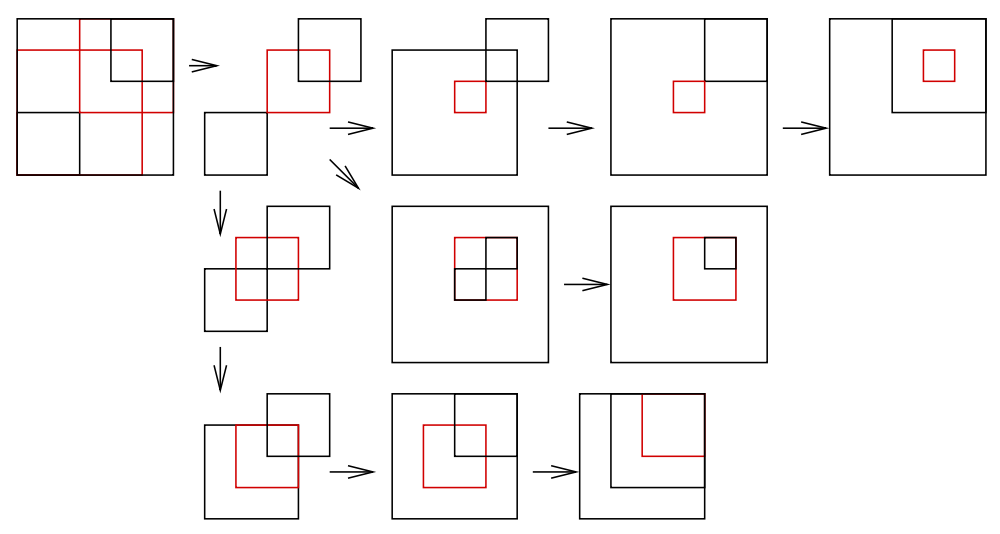

FiguRE 17. A sample calculation in $F(1,3 ; 5)$
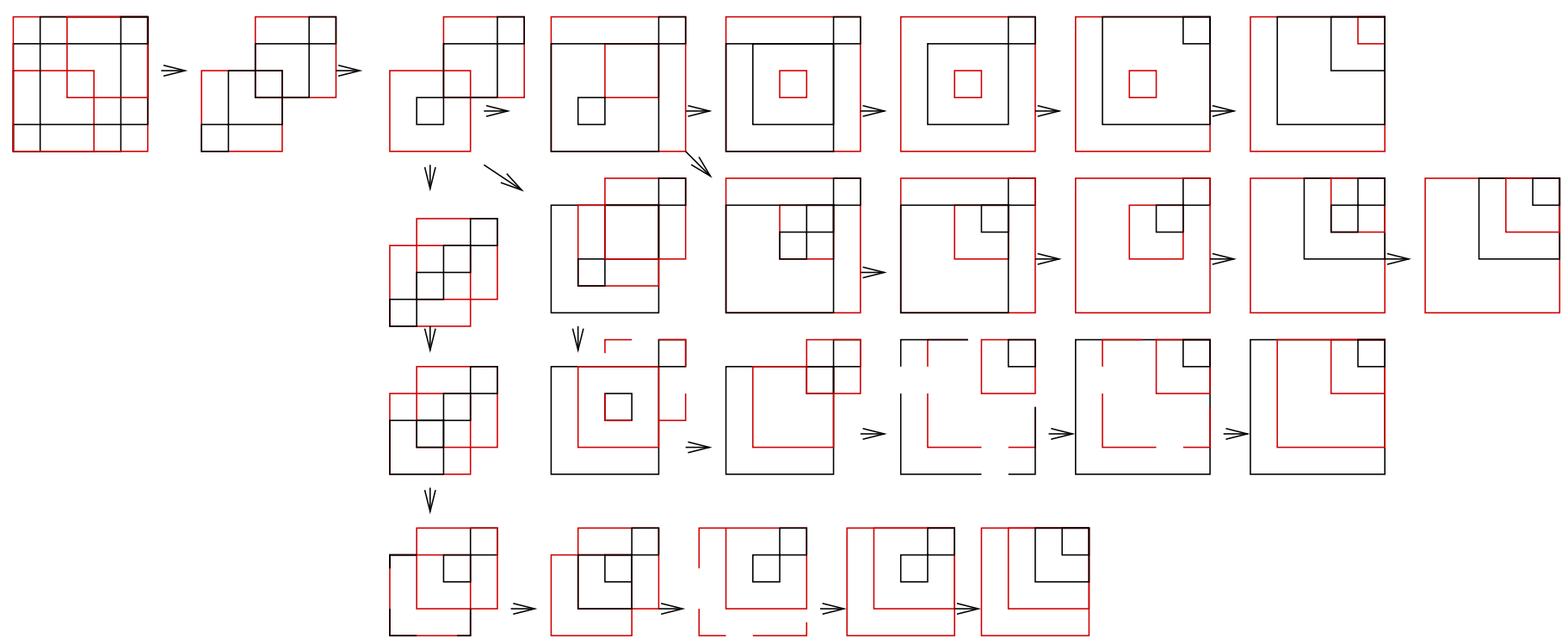

Figure 18. A sample calculation in $F(2,4 ; 5)$

Lemma 4.24. Let $M$ be a normalized painted Mondrian tableau. Let $S$ be the square defined by Rule 4.15. Then every square whose lower-left corner is southwest of $l(S)$ contains $S$.

Proof. Since every red square is a span of black squares, it suffices to check the lemma for black squares. Let $S^{\prime}$ be a black square with $l\left(S^{\prime}\right)<l(S)$. We can assume that $l\left(S^{\prime}\right)>l\left(T_{1}\right)$, otherwise $S^{\prime}$ would be nested. By Mon1b if $l \operatorname{ch}(S) \subset S^{\prime}$, then $S \subset S^{\prime}$. Hence, we may assume that $S^{\prime}$ has a gap contained in $l \operatorname{ch}(S)$. Since $l\left(S^{\prime}\right)<l(S)$, there must exist $T_{i}$ for $i<h$ that does not contain $S^{\prime}$. Suppose $T_{i_{0}}$ is the smallest index square that does not contain it. We must have $l\left(T_{i_{0}}\right)<l\left(S^{\prime}\right)$ by the order of degeneration. Hence the gaps of $S^{\prime}$ must be a subset of the gaps of $T_{i_{0}}$. This is a contradiction since $S$ is contained in $T_{i_{0}}$ and $S^{\prime}$ has a gap contained in $l c h(S)$. In the terminology of the previous section $S$ is a degeneratable square for the generalized Mondrian tableau consisting of the black squares of $M$.

Lemma 4.25. Let $M$ be a normalized, admissible painted Mondrian tableau. Let $S$ be determined by the Rule 4.15. Then $S$ can have at most two black neighbors. If $S$ has two black neighbors, then the smallest neighbor coincides with a red square. Any square of $M$ can have at most one red neighbor. 
Proof. In fact if $B_{1} \subset B_{2}$ are two black squares with $l\left(B_{2}\right)>l(S)$ and $B_{1} \not \subset S$, then $B_{1}$ must coincide with a red square. Otherwise, by APM2 there would exist a red square $R_{1}$ strictly contained in $B_{2}$ and containing $B_{1}$. If $B_{1}$ does not coincide with $R_{1}$, then by APM2 either $B_{1} \subset S$ or $S$ contains a filler $F$ with $l(F)<l\left(B_{1}\right)$. The first case is ruled out by assumption. The second case contradicts the definition of $S$. Hence, $B_{1}$ must coincide with a red square $R_{1}$. Suppose there were more than two black neighbors: $N_{1} \subset N_{2} \subset \cdots \subset N_{r}$. Then $N_{1}, \ldots, N_{r-1}$ would have to coincide with red squares. However, by APM1 the square $S$ would have to contain all of these red square but the one coinciding with $N_{r-1}$. This contradicts the definition of a neighbor. If a square $T$ of $M$ has more than one red neighbor $R N_{1} \subset R N_{2}$, then by APM1 the square $R N_{1}$ has to be contained in $T$ contradicting the definition of a neighbor. The lemma follows.

Lemma 4.26. Let $M$ be a normalized painted Mondrian tableau. Let $S$ be the square defined by Rule 4.15. Suppose $S \neq T_{1}$. Then either $S$ is a filler in $T_{h-1}$; or there exists a black square $B$ such that $l(B)>l(S)$, $B \not \subset S$ and $B \subset T_{h-1}$. Furthermore, in the latter case any square $S^{\prime}$ with $l\left(S^{\prime}\right)<l(S)$ contains $B$.

Proof. By assumption $T_{1} \neq S$. By Rule 4.15 either $T_{h-1}$ contains a filler $F$ or the squares contained in $T_{h-1}$ are not nested. Otherwise we would be moving $T_{h-1}$ instead of $S$. In the first case necessarily $l(F) \geq l(S)$. If $l(S)=l(F)$, then $S=F$ since $M$ is normalized. If $l(F)>l(S)$, then $F$ cannot be contained in $S$ since otherwise it would be a filler of $S$ contradicting Rule 4.15. If $T_{h-1}$ does not contain any fillers, then the squares in $T_{h-1}$ are not nested. Hence there exists $B \subset T_{h-1}$ with $l(B)>l(S)$. Since the squares contained in $S$ are nested $B$ cannot be contained in $S$. Finally, in case $S \neq F$, there remains to show $F$ or $B$ is contained in every square $S^{\prime}$ with $l\left(S^{\prime}\right)<l(S)$. We can assume $l\left(S^{\prime}\right)>l\left(T_{1}\right)$, otherwise the claim is trivial. Either $S^{\prime}$ is $T_{i}$ for some $i<h$ or there exists a minimal index $i_{0}$ such that $T_{i_{0}}$ does not contain $S^{\prime}$. We can assume we are in the latter case. We must have $l\left(S^{\prime}\right)>l\left(T_{i_{0}}\right)$. Hence the gaps of $S^{\prime}$ are a subset of the gaps of $T_{i_{0}}$. By Mon1b, every square contained in $T_{i_{0}}$ and whose lower-left corner is northeast of $l\left(S^{\prime}\right)$ is also contained in $S^{\prime}$.

Lemma 4.27. Let $M$ be a normalized, admissible painted Mondrian tableau. Let $S \neq T_{1}$ be the square determined by Rule 4.15. If $S$ is a filler in $T_{h-1}$, then $S$ has at most one black neighbor $N$ that does not coincide with a red square.

Proof. Suppose $l \operatorname{ch}(S)=\left\{e_{i}, \ldots, e_{j}\right\}$. Since $S$ is a filler, it shares a lower-left corner with a red square $R$. We can assume that $e_{j+1}$ is not a hole of $R$. Otherwise $S$ does not have any neighbors. Moreover, $e_{j+1}$ cannot be a gap of $R$. Otherwise $S$ and $R$ would coincide and $S$ could not be a filler by Lemma 4.23 . Since $S$ does not coincide with $R$, there must exist black squares in $R$ not contained in $S$. Let $B$ be the one with the southwest most lower-left corner. Note that $e_{j+1} \in B$. We know that there can be at most two black neighbors of $S$ and if there are two then the smaller one coincides with a red square. Let $N_{1} \subset N_{2}$ be the two neighbors. If $l(B)<l\left(N_{1}\right)$, then we have a contradiction unless $N_{2}=B$. But in that case $N_{1}$ which coincides with a red square $R_{1}$ is contained in $R$. Hence by APM1, $N_{1} \subset R_{1} \subset S$ contradicting the definition of a neighbor. We can assume $l(B)>l\left(N_{1}\right)$. Then the gaps of $N_{1}$ are a subset of the gaps of $B$. If $B$ has a gap, then $N_{1}, S$ and $B$ agree northeast of the gap. We again conclude that $N_{1} \subset R_{1} \subset R$ contradicting APM1. So there can be at most one neighbor. In fact it has to coincide with $B$ and by APM1 cannot coincide with a red square.

Lemma 4.28. Let $M$ be a normalized, painted Mondrian tableau. Let $S$ be the square determined by Rule 4.15. Suppose $S \neq T_{1}$ is not a filler in $T_{h-1}$. Let $N$ be the minimal (with respect to inclusion) black square not contained in $S$ with $l(N)>l(S)$ such that any square $S^{\prime}$ with $l(S)<l\left(S^{\prime}\right)<l(N)$ is either contained in $S$ or contains $N$. Then $N \subset T_{h-1}$. In particular, if $S$ has two black neighbors, then the smaller one is contained in every square $S^{\prime}$ with $l\left(S^{\prime}\right)<l(S)$.

Proof. By Lemma $4.26 T_{h-1}$ contains a square $B \not \subset S$ with $l(B)>l(S)$. If $l(B) \leq l(N)$, then $N \subseteq B \subset T_{h-1}$ by assumption. If $l(B)>l(N), N \subset T_{h-1}$ by Mon1b and the minimality of $N$.

The following observation will play a crucial role in the dimension calculations. 
Proposition 4.29. Let $M$ be a normalized, painted Mondrian tableau. Let $S$ be the square of $M$ determined according to Rule 4.15. If the length of intersection of a red square $R$ with another red square increases when moving $S$ according to 4.16, then either $S$ coincides with $R$ or there exists a red square $R^{\prime}$ not contained in $R$ with $l\left(R^{\prime}\right)=l(S)$.

Proof. Suppose $l \operatorname{ch}(S)=\left\{e_{l}, \ldots, e_{j}\right\}$. If all the red squares containing $S$ also contain $e_{j+1}$, then the length of the intersection of two red squares do not increase. However, there may be a red square $R$ containing $S$ that does not contain $e_{j+1}$. If $e_{j+1}$ is a hole of $R$, then the move cannot increase the length of intersection of $R$ with any red square since no red square $R^{\prime}$ with $l\left(R^{\prime}\right)>l(R)$ can contain $e_{j+1}$. By PM3a, we can assume that $e_{j+1}$ is a gap of $R$ which is not a hole. If $l(S)=l(R)$, then $S$ contains any square contained in $R$ by Mon1b and PM3c. Since $R$ is the span of the black squares it contains by PM2, $R$ must coincide with $S$. Otherwise, $l(R)<l(S)$. Let $B$ be the square with $l(B)=l(R)$. Let $T_{i_{0}}$ for $1 \leq i_{0}<h$ be the square with largest index such that $l\left(T_{i_{0}}\right) \leq l(B)$. Either $B=T_{i_{0}}$; or the gaps of $B$ are a subset of the gaps of $T_{i_{0}}$. In either case $e_{j+1}$ is a gap of $T_{i_{0}}$. Hence, $S, B$ and $T_{i_{0}}$ agree northeast of $e_{j+1}$ by Mon1b. Hence $T_{h-1}$ agrees with $S$ northeast of $e_{j+1}$. By Rule 4.16 there cannot be any squares $S^{\prime} \subset T_{h-1}$ with $l\left(T_{h-1}\right)<l\left(S^{\prime}\right)<l(S)$. Hence any square contained in $T_{h-1}$ is also contained in $S$ by Mon1b. By Lemma $4.26 S$ must be a filler in $T_{h-1}$. The red square $R^{\prime}$ with $l\left(R^{\prime}\right)=l(S)$ is the red square in the lemma.

Observe that the tableau $M^{\prime}$ formed after moving $S$ is not necessarily a painted Mondrian tableau. The Conditions Mon2 and Mon3 do not have to be satisfied for the black squares in $M^{\prime}$. Nevertheless, define the virtual dimension of $M^{\prime}$ by associating to it the same quantity as in Definition 4.12 .

Lemma 4.30. The virtual dimension of $M^{\prime}$ is less than or equal to the dimension of $M$. The dimension of the normalization of $M^{\prime}$ is less than or equal to the dimension of $M$.

Proof. The sum of the side-lengths of the black squares in $M$ and $M^{\prime}$ are equal. If $S(0)$ does not contain the smallest black neighbor of $S$ and $S(0)$ is not contained in the largest black neighbor of $S$, then the number of containment relations among the squares in $M$ and $M^{\prime}$ are also equal. Hence the virtual dimension of $M^{\prime}$ is equal to the dimension of $M$.

By Condition 2 in the definition of normalization, $S(0)$ can contain at most one of the black neighbors of $S$. If $S(0)$ contains the smallest black neighbor $N$, then every square whose lower-left corner is (non-strictly) southwest of $l(S)$ and did not contain $N$ also contains $N$. This increases the number of containment relations among the black squares by the number of such squares. On the other hand, the number of black squares that are contained in red squares increases by the number among them which are red. If $N$ coincides with a red square, the number of containment relations among the red squares increases by the same amount. Hence, the virtual dimension of $M^{\prime}$ strictly decreases except when the following are satisfied:

(1) $N$ does not coincide with a red square; and

(2) Every square whose lower-left corner has index less than or equal to $l(S)$ and does not contain $N$ is red.

Note that in particular these conditions imply that $S$ should coincide with a red square. Hence, $S$ is not a filler and by Lemma 4.26 the squares with lower-left corner strictly southwest of $l(S)$ contain $N$.

If $S(0)$ is contained in the largest black neighbor $N$ of $S$, then the number of containment relations among black squares increases by one. If $l(N)$ is the lower-left corner of a red square, then the total number of black squares in red squares increases by one. If in addition $l(S)$ is the lower-left corner of a red square, then the number of containment relations among the red squares increases by one. Hence, the virtual dimension of $M^{\prime}$ is strictly smaller than the dimension of $M$ except when $S$ does not coincide with a red square and $l(N)$ is the lower-left corner of a red square. Observe that it is not possible for $S(0)$ to be contained in a red neighbor $R N$ of $S$ where the black square $B$ that has the same lower-left corner as $R N$ is contained in $S$. $B$ would be a filler for $S$ contradicting Rule 4.15. This is the main reason for choosing the degeneration order in Rule 4.15. It follows that the virtual dimension of $M^{\prime}$ is at most equal to the dimension of $M$.

If $l(S)$ is the lower-left corner of a red square $R$, then we shrink $R$ by one unit. This either preserves the virtual dimension or decreases it in case the lower-left corner of $R$ coincides with the lower-left corner of a red square not contained in $R$. 
Next we check that normalizing does not increase the virtual dimension of the tableau. We will say that a square $U^{\prime}$ is a shrinking of a square $U$ in $M^{\prime}$ if $U^{\prime}$ is obtained by shrinking the lower-left corner of a square in $M^{\prime}$. Since $M$ is normalized, at each stage of the normalization process for $M^{\prime}$ at most two black squares and two red squares can share the same lower-left corner. If $T$ is a square of the tableau, denote by $T^{\prime}$ the square $T-l(T)$. If a black square $T$ has the same lower-left corner as a square it contains, we shrink its lower-left corner by one. Suppose $T^{\prime}$ is not contained in any new squares. Then this operation decreases containment relations among black squares by one and the total side-length of black squares by one. It does not change containment relations among red squares or black squares and red squares. Hence, it preserves virtual dimension. Suppose $T^{\prime}$ starts being contained in $b$ black squares and $r$ red squares. Then this changes the virtual dimension by $r-b$. We note that each time $T^{\prime}$ is contained in a red square $R$, it must also be contained in the black square $B$ whose lower-left corner is the same as $R$. We already saw this for the square $S$. Suppose $T^{\prime}$ is not a shrinking of $S$, but some other black square $T^{\prime \prime}$ necessarily with $l\left(T^{\prime \prime}\right)>l(S)$ in $M$. If $B$ were properly contained in $T^{\prime}$, then $B$ would be strictly contained in $T^{\prime \prime}$ in $M$. Hence by APM2 for $M, B$ would be contained in a red square $R^{\prime}$ contained in $T$ and containing $B$. $B$ cannot coincide with $R^{\prime}$ since then $R$ and $R^{\prime}$ would have the same lower-left corner contradicting that $M$ is normalized. But then by APM2 either $S$ would contain $B$ or a filler $F$ with $l(F)<l(B)$. Both are contradictions since either $F$ or $B$ would be a filler in $S$. Finally, normalizing the red squares clearly preserves or decreases virtual dimension. No new black squares can be contained in a red square during the normalization. One fewer black square can be contained in it exactly when one containment relation among the red squares is eliminated. Note that in case a red square starts being contained in a red square that it was not previously contained in prior to normalization, then the virtual dimension strictly decreases.

Finally, if $S(0)$ contains the smallest black neighbor of $S$ and has the same lower-left corner as a square contained in $S$, then we need to shrink the upper-right corner of $l \operatorname{ch}\left(S(0)^{\prime}\right)$ (the shrinking of $\left.S(0)\right)$ by one unit. Let $S=S_{1}, \ldots, S_{r}$ be the squares with the property that the index of $l\left(S_{i}\right)$ is one larger than the index $l\left(S_{i-1}\right)$ and $S_{i} \subset S$. Then the total side-length of black squares decreases by one. The number of containment relations among black squares increases by $r-2$. The number of black squares contained in red squares increases by the number of red squares among $S_{2}, \ldots, S_{r}$. Containment relations among red squares do not change. Hence the dimension stays constant or decreases. Of course, if at any stage the side-length of a square shrinks to zero we discard the tableau and assign to it dimension -1 . We leave it to the reader to formulate the cases when the dimension stays constant. We will summarize this below.

We will use the following observations to verify that the tableaux $M_{0}, M_{1}(N R), M_{2}\left(N_{i}\right)$ and $M_{3}(N R)$ are normalized, admissible painted Mondrian tableaux.

Observation 4.31. If after moving $S$ a red square $R(0)$ of $M^{\prime}$ has a new gap, then $R(1)$ in $M$ contains $S$ and has $e_{j+1}$ as a gap. The new gap of $R(0)$ is $l(S)$. Since every square with lower-left corner southwest of $l(S)$ contains $S$, there can be no red squares contained southwest of $l(S)$. Hence PM3a for $M$ implies PM3a holds for $M^{\prime}$. PM3c holds for $M^{\prime}$ since any square with lower-left corner southwest of $l(S)$ contains $S$ and PM3c holds for M. PM3b holds for the squares in $M^{\prime}$ that have $l(S)$ as their new gap and the gap $l(S)$. However, some red squares that had $e_{j+1}$ may no longer have it as a gap. We note that a red square $R^{\prime}(1)$ with $l(S)<l\left(R^{\prime}(1)\right)$ in $M$ that has $e_{j+1}$ as a gap must be contained in every red square whose lower-left corner is southwest of $l\left(R^{\prime}(1)\right)$ by PM3b for $M$. It is easy to see that PM3b also holds for $M^{\prime}$.

Observation 4.32. Normalization of $M^{\prime}$ preserves the properties PM3a, b, c. Note that normalization either preserves or eliminates the gaps of a red square. Similarly, it either preserves holes or may turn holes which were also gaps into gaps. As in the proof of Lemma 4.30 if shrinking a black square $T$ results in $T$ sharing the lower-left corner with a black square $B$ and a red square $R$, then either $T^{\prime}$ contains $R$ or is contained in $B$. In the second case a hole or a gap of $R$ is clearly preserved. In the first case, if $T^{\prime}$ contains a hole which is not a gap, then the squares contained in $R$ cannot be nested. Since by APM2 the square $S$ must contain $R$, this contradicts Rule 4.15. Shrinking the lower-left corner $l(B)$ for a black square $B \subset R$ whose lower-left corner agrees with $l(R)$ for a red square does not alter the holes and gaps of $R$. It follows that PM3a and PM3c hold for the normalization of $M^{\prime}$. The proof of PM3b is analogous to the proof of Lemma 3.41, hence left to the reader. 
Observation 4.33. Suppose $S(0)$ does not contain the smallest neighbor of $S$, then APM1, APM2 and APM3 hold for $M^{\prime}$. This is clear in case $S$ is not contained in its largest neighbor since none of the containment relations among the squares change. If $S$ is contained in its largest neighbor, since $S$ is contained in every square whose lower-left corner is southwest of $l(S)$, APM1, APM2 and APM3 hold in that case as well.

Observation 4.34. If the normalization of $M^{\prime}$ has the same dimension as $M$, then it satisfies APM1, APM2 and APM3. $M^{\prime}$ is not normalized when $S(0)$ contains the smallest neighbor of $S$ or $S(0)$ shares a lower-left corner with another square in $M^{\prime}$. Otherwise shrinking the red squares so that they are the spans of black squares contained in them, turns $M^{\prime}$ to a normalized tableau. Hence, APM1, APM2 and APM3 are satisfied by the previous observation. If $S(0)$ contains the smallest neighbor $N$ of $S$, we can assume that $N$ does not coincide with a red square, $S(0)$ coincides with a red square $R(0)$ and that the normalization of the lower-left corner of $S(0)$ remains strictly southwest of $l(N)$. Otherwise by Lemma 4.30 the dimension of the normalization of $M^{\prime}$ is strictly less than the dimension of $M$. In that case, after shrinking the lower-left corner of $S(0)$ and the upper-right corner of $l \operatorname{ch}\left(S(0)^{\prime}\right)$, we obtain a black square which is contained in every square whose lower-left corner is southwest of it. The shrinking of the red square $R(0)$ is not contained in the squares that were contained in $R(0)$, but is contained in every square whose lower-left corner is southwest of $l(R(0))$. Finally, $N$ is not contained in any new black squares unless it is contained in $S(0)$ and $S(0)$ does not share a lower-left corner with any squares strictly contained in it. In that case $N$ is contained in every square whose lower-left corner is southwest of $S(0)$. Hence APM1, APM2 and APM3 hold for the normalization of $M^{\prime}$. If $S(0)$ shares a lower-left corner with a square strictly contained in it, but does not contain its smallest neighbor, then when we normalize the lower-left corners it is clear that APM1, APM2 and APM3 are preserved unless $S(0)^{\prime}$ starts sharing a lower-left corner with its largest neighbor $N^{\prime}$. By Lemma 4.30 and APM1, $S$ can have only one neighbor $N_{1}$ since $N_{1}$ has to be the lower-left corner of a red square. We then shrink the lower-left corner of $N_{1}$. If the shrinking of $N_{1}$ shares a lower-left corner with a square then either that square is a square contained in $S(0)$ or is a neighbor of $N_{1}$. As in Lemma 4.30 if $N_{1}$ starts sharing the lower-left corner with its largest neighbor, then either the dimension strictly decreases or $N_{1}$ has a unique neighbor $N_{2}$ whose lower-left corner is the lower-left corner of a red square. Continuing we see that there are no new containment relations among the black squares or among the red squares in the normalization that did not hold in $M^{\prime}$. If the shrinking of a black square $B$ starts being contained in a red square $R$, then $B$ is either $S(0)$ or it is also contained in a red square whose lower-left corner is southwest of $l(R)$. Note that any black square $\tilde{B}$ containing $R$ must have its lower-left corner strictly southwest of $l(B)$. It is now easy to see that APM1, APM2 and APM3 are preserved in this case as well.

Lemma 4.35. Whenever Algorithm 4.19 produces the tableau $M_{0}, M_{0}$ is a normalized, admissible painted Mondrian tableau.

Proof. $M_{0}$ is normalized by definition. Mon1a and Mon1b hold by the proof of Lemma 3.41 when normalizing lower-left corners. As in the proof of Lemma 4.30, Condition 2 in Definition 4.6 fails only when $S(0)$ contains its smallest neighbor. In that case, $S(0)^{\prime \prime}$ obtained by shrinking the upper-right corner of $l \operatorname{ch}\left(S(0)^{\prime}\right)$ is contained in every square whose lower-left corner is southwest of it. Hence, Mon1a and Mon1b hold. PM2 holds by construction. It now follows that PM1, PM2, PM4, PM5 hold. The proof of PM3a, b, c follow from the two Observations 4.31 and 4.32 above. Finally APM1, APM2 and APM3 hold by Observations 4.31 and 4.32.

Lemma 4.36. Whenever Algorithm 4.19 produces the tableau $M_{1}(N R)$, it is a normalized, admissible painted Mondrian tableau of dimension equal to the dimension of $M$.

Proof. $M_{1}(N R)$ is already normalized. The lower-left corners of the squares in $M_{1}(N R)$ are the same as the lower-left hand corners of the squares in $M$, hence the lower-left corners of any two squares of the same color in $M_{1}(N R)$ are distinct. Condition 2 in the Definition 4.6 holds even when $S(0)$ contains the smallest neighbor of $S$. Next we claim that the squares $B_{i_{2}}, \ldots, B_{i_{j-1}}$ are squares with lower-left corner between $l(S)$ and $l(R)$ and the span replaces the lower-left chops of the consecutive ones by the spans of their lower-left chops. Furthermore, that span becomes the lower-left chop of the squares $B_{i_{h}, i_{h+1}}$. Suppose $B_{i_{h}}$ has a gap between $l(S)$ and $e_{j+1}$, then by Mon1b $B_{i_{h}} \subset S$. But then $B_{i_{h}}$ cannot contain $e_{j+1}$, leading to a contradiction. The lower-left chops of two consecutive squares necessarily intersect (since they all contain $e_{j+1}$ ). In addition to the squares formed 
by the spans of $S(1)$ and $B_{i_{2}}$ and the spans $B_{i_{h}}$ and $B_{i_{h+1}}, M_{1}(N R)$ contains the new squares $I_{B}=S(0) \cap N R$ in black and $I_{R}=R(0) \cap N R$ and $U_{R}=R(1) \cup N R$ in red. In particular, the gaps of the new squares are all gaps of the squares in $M^{\prime}$, except possibly for the gaps of $I_{B}$ and $I_{R}$.

We first check Mon1a. Suppose a black square is contained entirely to the southwest of a gap. This cannot be any of the new spans, since $S$ would be contained southwest of that gap in $M$. We need to verify Mon1a for $I_{B}$. Let $g$ be a gap northeast of $I_{B}$. Since in $M, S$ cannot be contained southwest of $g$, $S$ must contain basis elements northeast of $g$. But then the black square $B$ that has the same lower-left corner as $N R$ must also contain such basis elements by Mon1b for $M$. Since the gaps $B$ are a subset of the gaps of $S, I_{B}$ cannot be contained strictly southwest of $g$. The verification of Mon1b is similar to the proof of Lemma 3.43. The arguments for the red squares $U_{R}$ and $I_{R}$ are also similar and are left to the reader. It is then easy to see that PM1, PM2, PM3a,b,c, PM4 and PM5 hold for $M_{1}(N R)$.

It suffices to verify APM1, APM2 and APM3 with respect to the newly formed squares. The square $I_{R}$ is contained in every square whose lower-left corner is southwest of $U_{R}$ since $I_{R}$ is contained in $S(0)$ and $U_{R}$ has the same lower-left corner as $S(1)$. By the definition of a neighbor and APM1 for $M$, no red squares $R^{\prime}$ with $l\left(U_{R}\right)<l\left(R^{\prime}\right)<l\left(I_{R}\right)$ can contain $I_{R}$. Furthermore all such squares are contained in every square whose lower-left corner is southwest of $U_{R}$ since in the tableau $M$ they were contained in $S$. No red square that did not contain both $R(1)$ and $N R$ contains the union $U_{R}$. Hence if $R^{\prime \prime}$ is a square containing $U_{R}$ and $\tilde{R}$ is a red square with $l(\tilde{R})<l\left(R^{\prime \prime}\right)$, then $U_{R} \subset \tilde{R}$ since $\tilde{R}$ had to contain both $R(1)$ and $N R$. It follows that APM1 holds for $M_{1}(N R)$.

$I_{B}$ coincides with a red square. Note that if a black square $B$ contains one of the spans of the squares $B_{i_{h}, i_{h+1}}$ for $1 \leq h<j$, then $B$ must contain both $B_{i_{h}}$ and $B_{i_{h+1}}$. Hence, $B$ must strictly contain a red square containing $B_{i_{h}}$ and $B_{i_{h+1}}$ by APM2 for $M^{\prime}$. This red square cannot have its lower-left corner between $l(R(1))$ and $l(N R)$ by the definition of a neighbor and APM1 for $M^{\prime}$. Hence the same red square strictly contained in $B$ contains the union. When $h>1$, the second condition for APM2 follows for this red square by the same condition for the red square in $M^{\prime}$ and the square $B_{i_{h}}$. Since $S$ coincides with a red square by Lemma 4.23 it is not a filler. Hence by Lemma 4.28 every square whose lower-left corner is southwest of $l(S)$ contains $B_{i_{2}}$ since this is the smallest neighbor of $S$. Hence the second condition in APM2 holds as well. APM3 holds for $U_{R}$ since if there is a black square strictly containing $U_{R}$, that black square must contain all $B_{i_{h}}$. Hence, by APM2 it must strictly contain a red square containing them. Since the lower-left corner of this red square must be southwest of $l(S)$, APM3 holds for $U_{R}$ by APM3 for $M$. If there is a black strictly contained in $I_{B}$ or $I_{R}$, then it is contained in every square with lower-left corner southwest of $I_{B}$. Similarly, for any black squares strictly contained in $B_{h, h+1}$ except for $I_{B}$. For all other squares APM2 and APM3 follow from APM2 and APM3 for $M^{\prime}$.

Finally, we compute the dimension. Applying the construction of $M_{1}(N R)$ increases the total side-length of the black squares by one. The number of containment relations among the black squares increases by $j-1$. The number of black squares contained in red squares increases by $j-1$ and the number of containment relations among red squares increases by 1 . Hence $M_{1}$ and $M$ have the same dimension.

Lemma 4.37. Whenever Algorithm 4.19 produces the tableau $M_{2}\left(N_{i}\right)$, it is normalized and admissible.

Proof. By the analysis for Corollary 3.44, the total side-length of the black squares and the number of containment relations among the black squares each increase by one unless $S(0)$ contains the smallest neighbor $N_{1}$ of $S$ in $M_{2}\left(N_{2}\right)$. In the latter case the number of containment relations among black squares increases by two. The number of black squares contained in each red square remains constant. The number of containment relations among red squares is either constant or increases. Hence if $S$ has two black neighbors $N_{1} \subset N_{2}$ and $S(0)$ contains $N_{1}$, then $M_{2}\left(N_{2}\right)$ has strictly smaller dimension. If both $S$ and $N_{1}$ coincide with red squares, then the number of containment relations among red squares increases by at least one. Hence the dimension of $M_{2}\left(N_{1}\right)$ is strictly smaller than that of $M$. Similarly, suppose $S$ coincides with a red square and has only one black neighbor $N_{1}$ with the property that $l\left(N_{1}\right)=l(R)$ for some red square containing $N_{1}$. If $S$ does not contain any black squares that are not contained in $N_{1}$ and do not coincide with a red square, then $M_{2}\left(N_{1}\right)$ has strictly smaller dimension. In all other cases the dimension of $M_{2}\left(N_{i}\right)$ is at most the dimension of $M$.

The generalized Mondrian tableau formed by the black squares is normalized and satisfies Mon1a and Mon1b by Lemma 3.41. If the lower-left chop of a red square $R$ coincided with the lower-left chop of the square $S$, we 
might need to shrink the lower-left corner of $R(0)$. As a result of this the lower-left corner of $R(0)$ might coincide with the lower-left corner of a red square. Hence, the red squares may need to be normalized. Similarly if the neighbor $N_{i}$ has the same lower-left corner as a red square $R^{\prime}$, we might need to shrink $R^{\prime}$. This might introduce a hole in $R^{\prime}$. Normalizing the red squares does not change Mon1a and Mon1b. To preserve the dimension, when normalizing red squares, the shrinking $R(0)^{\prime}$ of $R(0)$ cannot start sharing a lower-left corner with a red square not contained in it. Since the red squares contained in $R(0)$ are nested and contained in $S(0)$, they are contained in every square whose lower-left corner is southwest of their lower-left corners. The tableaux $M_{2}\left(N_{i}\right)$ satisfy PM2 by construction. The proof of the properties PM3a, b, c are as in Observation 4.32. Finally since $M_{2}\left(N_{i}\right)$ is normalized PM4 and PM5 hold.

The proofs of APM1, APM2 and APM3 are similar to the previous cases. Note that in these tableaux whenever they have the same dimension as $M$, there are no new containment relations among the red squares. As observed any containment relations among squares that no longer hold happens because the shrinking $R(0)^{\prime}$ does not contain some of the red squares originally contained in it. But these squares are contained in every square whose lower-left corner is southwest of their lower-left corner. Hence APM1 for $M$ implies APM1 for $M_{2}\left(N_{i}\right)$.

It suffices to check APM2 with respect to $I_{B}=S(0) \cap N_{i}$ and $U_{B}=S(1) \cup N_{i}$. Since $I_{B} \subset S(0)$ if $I_{B} \subset B^{\prime}$, then every square $B^{\prime \prime}$ with $l\left(B^{\prime \prime}\right)<l\left(B^{\prime}\right)$, then $I_{B} \subset B^{\prime \prime}$. Since the squares contained in $S$ are totally ordered, and $I_{B} \subset S(0)$, any square strictly contained in $I_{B}$ is contained in every square whose lower-left corner is southwest of $l\left(I_{B}\right)$. If $U_{B} \subset B^{\prime}$, then $B^{\prime}$ in $M^{\prime}$ contains both $S(0)$ and $N_{i}$. If $S$ has a black neighbor $N_{i}$ that coincides with a red square, then both $S$ and $N_{i}$ are contained in $T_{j-1}$, hence in every square whose lower-left corner is southwest of $l(S)$. We can assume that $N_{i}$ does not coincide with a red square. The same argument applies if there are no squares strictly contained in $N_{i}$ that are not contained in $S$ and $S$ is not a filler. We can assume that either $S$ is a filler in $T_{j-1}$ and $T_{j-1}$ does not contain $N_{i}$; or $N$ contains a red square not contained in $S$ and $T_{j-1}$ does not contain $N_{i}$. In the first case, there has to be a red square strictly contained in $B^{\prime}$ that contains $N_{i}$. It follows that $B^{\prime}$ must contain the red square $R$ with $l(R)=l(S)$. Note that $R$ also contains $U_{B}$. In the second case by APM1 for $M$, there cannot be a red square with lower-left corner between $l(S)$ and $l\left(N_{i}\right)$ containing $N_{i}$, hence the red square containing $N_{i}$ and contained in $B^{\prime}$ must also contain $S$ and hence $U_{B}$. Either $N_{i}$ coincides with the red square northeast of $l\left(N_{i}\right)$ in which case so does $U_{B}$. Else both $N_{i}$ and $S$ need to be contained in a red square with lower-left corner southwest of $l(S)$. Hence so does $U_{B}$. APM2 and APM3 follow from these observations.

Lemma 4.38. Whenever Algorithm 4.19 produces the tableau $M_{3}(N R)$, it is normalized and admissible.

Proof. Note that the tableau consisting of the black squares is already normalized. If $S$ coincides with a red square, however, we might need to normalize the red squares of the tableau. Prior to the normalization it is easy to calculate the virtual dimension of the tableau. Assuming $S(0)$ does not contain its neighbor, the total side-length of the black squares (if the tableau is non-empty) increases by one. The total containment relations among them increases by $l+1$. On the other hand, the total number of black squares contained in red squares increases by $l$. In case $S$ coincides with a red square $R$, then we need to shrink $R$ so that it is the span of the black squares contained in it. As in the previous cases this process decreases the number of containment relations among red squares by an amount equal to the number of black squares contained in the shrinking of $R(0)$. Unless $R(0)$ is contained in the neighbor $B_{1}$ which coincides with $R_{1}$. In that case normalizing strictly decreases the dimension. Hence in case $S$ coincides with a red square, for this tableau to have the same dimension as $M$ there must exist a black square contained in $R(0)$ but not in $R_{1}$ and which does not coincide with a red square. Similarly it is easy to see that if $S(0)$ contains the smallest neighbor $N_{1}$ of $S$, the dimension of Tableau $M_{3}(N R)$ is strictly smaller than the dimension of $M$.

The verification of the properties Mon1a, Mon1b, PM2, PM3a,b,c, PM4 and PM5 is analogous to the previous cases. We leave this to the reader. Here we verify APM1, APM2 and APM3. APM1 is clear from APM1 for $M^{\prime}$ since the containment relations among the red squares do not change unless $S$ coincides with a red square $R$. By the dimension discussion, the red squares that $R(0)$ coincides with during the shrinking have to be all contained in $S$. These squares do not contain the shrinking $R(0)^{\prime}$ of $R(0) . R(0)^{\prime}$ is contained in every square whose lower-left corner is southwest of $l(S)$. Finally any red square contained in $R(0)^{\prime}$ is contained in every square whose lower-left corner is southwest of $R(0)^{\prime}$ since these squares were contained in $S$ and the squares 
in $S$ were nested. The squares $R_{i}$ are not contained in any red squares that they were not contained in (even if they acquire holes). From these observations APM1 for $M$ implies APM1 for $M_{3}(N R)$. APM2 is clear with respect to the squares formed by the intersections $S(0) \cap B_{1}$ and $B_{h} \cap B_{h+1}$ since any black square containing one of the intersections either contains the other one as well, hence contains the red square $R_{h}$. If a square $B$ contains $B_{h} \cap B_{h+1}$ and a square $B^{\prime}$ with $l\left(B^{\prime}\right)<l(B)$ does not contain it, then $B^{\prime}$ must still contain a square since $B^{\prime}$ by construction cannot have lower-left corner northeast of $l(S(0))$. Hence $B^{\prime}$ must contain at least $S(0) \cap B_{1}$. Suppose $R_{p}$ is the first red square among $R_{1}, \ldots, R_{l}$ that $B^{\prime}$ does not contain. Then $B_{p-1} \cap B_{p}$ is a filler in $B^{\prime}$. We need to verify APM2 also with respect to the square $U$ formed by the span of $S(1), B(1), \ldots, B_{l}$. The black squares contained in $U$ are either of the form $B_{h} \cap B_{h+1}$ or they are squares contained in $S(0)$. The latter squares are contained in every square whose lower-left corner is southwest of $l(U)=l(S(1))$. The squares $B_{h} \cap B_{h+1}$ are contained in the squares $R_{h}$ contained in $U$. Suppose a black square $B$ contains $U$. Then $B$ contains $B_{l+1}$. Hence $B_{l+1}$ has to be contained in a red square strictly contained in $B$. This red square either has southwest corner northeast of $l\left(B_{l+1}\right)$ or southwest of $S(0)$. In the former case by APM3 for $M$, every square whose lower-left corner is southwest of $l(B)$ must contain $B_{l+1}$, hence by Mon1b also $U$. In the latter case, there exists a red square strictly contained in $B$ that contains $U$. Hence APM2 holds for the tableau $M_{3}(N R)$. It suffices to verify APM3 for the squares $R_{1}, \ldots, R_{l}$. In $R_{1}, S(0) \cap B(1)$ is contained in every square whose lower-left corner is southwest of any black square that contains $R_{1}$. For $R_{h}$ with $h>1$, the black squares that do not coincide with $R_{h}$ northeast of their lower-left corner are either contained in $S(0)$ (by APM1 and APM2 for $M$ ) or are of the form $B_{h-1} \cap B_{h}$. The former are contained in every square whose lower-left corner is northeast of $U$. The latter are contained in $R_{h-1}$. From these observations it is easy to verify APM3.

Remark 4.39. We can summarize the dimension discussion above as follows:

First suppose that the square $S$ coincides with a red square $R$. Then:

- If either $R(0)$ contains the red neighbor $N R$ of $R$ or $|R-N R|=\#_{R}(R \cup N R)(M)-\#_{R} N R(M)$, then only $M_{1}(N R)$ will have the same dimension as $M$.

- If $S$ has two black neighbors $N_{1} \subset N_{2}$ where the smaller $N_{1}$ coincides with a red square, then $M_{2}\left(N_{1}\right)$ has strictly smaller dimension than $M$. Suppose $S$ has only one black neighbor $N_{1}$ and there exists a red square $R^{\prime}$ such that $l\left(N_{1}\right)=l\left(R^{\prime}\right)$. If $S$ does not contain a black square $B$ such that $B$ does not coincide with a red square and is not contained in $N_{1}$, then $M_{2}\left(N_{1}\right)$ has smaller dimension than $M$.

- $M_{3}(N R)$ will have strictly smaller dimension than $M$ if there does not exist a black square that does not coincide with a red square and whose lower left corner is strictly between that of $R$ and $N R$.

Next suppose that the square $S$ does not coincide with a red square. Then if the smallest black neighbor $N_{1}$ of $S$ is contained in $S(0)$, then only $M_{2}\left(N_{1}\right)$ has the same dimension as $M$.

Next suppose $S(0)$ contains the smallest neighbor $N_{1}$ of $S$. If $M_{0}$ has the same dimension as $M$, then $S(0)$ must coincide with a red square $R, N_{1}$ cannot coincide with a red square and the largest black square $B$ contained in $R$ and not coinciding with a red square has the property $|R|-|B|>\#_{B} R(M)-\#_{B} B(M)$.

Finally, suppose the largest black neighbor $N$ of $S$ has the property that $|S-N|=\#_{B}(S \cup N)(M)-\#_{B} N(M)$. Consider the longest interval of uninterrupted set of basis elements that are lower-left corners of black squares, starting with $l(N)$ and proceeding northeast. Let $l(N), e_{i_{p}}, \ldots, e_{i_{q}}$ be the basis elements among these that are those which are not the lower-left corners of black squares strictly contained in $N$. Then $M_{0}$ has strictly smaller dimension than $M$ unless $l(N), e_{i_{p}}, \ldots, e_{i_{q}}$ are also the lower-left corners of red squares.

Remark 4.40. Note that it follows from the previous remark that at each stage an admissible painted Mondrian tableau occurring while running Algorithm 4.19 is replaced by at most three new tableaux. First suppose $S$ coincides with a red square. Then either the largest (and only) neighbor of $S$ coincides with a red square. In this case $M$ may be replaced by $M_{0}, M_{1}(N R)$ and $M_{3}(N R)$. If the largest neighbor $N$ of $S$ does not coincide with a red square, then $M$ may be replaced by $M_{0}, M_{1}(N R)$ and $M_{2}(N)$. Now assume that $S$ does not coincide with a red square. If the largest (and only) neighbor $N$ of $S$ coincides with a red square, then $M$ may be replaced by $M_{0}, M_{2}(N)$ and $M_{3}(N R)$. If the largest neighbor does not coincide with a red square, then $M$ may be replaced by $M_{0}, M_{2}\left(N_{1}\right)$ and $M_{2}\left(N_{2}\right)$. In each case we see that there are at most three tableaux. 
Proposition 4.41. The Algorithm 4.18 at each step replaces normalized, admissible painted Mondrian tableaux by normalized, admissible painted Mondrian tableaux and terminates with a collection of tableaux associate to Schubert varieties.

Proof. The proposition follows from Lemmas 4.35, 4.36, 4.37, 4.38. The fact that the algorithm terminates is clear. In each tableau that we replace $M$ with, the lower-left hand corners either stay the same or move strictly northeast. In Tableau $M_{0}$ there is at least one square whose lower-left corner moves strictly northeast. In the others, the total side-length of the black squares increases. Since these tableaux are contained in an $n \times n$ grid, these operations cannot go on indefinitely.

4.3. The geometry of painted Mondrian tableau. In this subsection we associate an irreducible subvariety of $F\left(k_{1}, k_{2} ; n\right)$ to every painted Mondrian tableau $M$. Then interpreting the moves on painted Mondrian tableau as a degeneration, we prove Theorem 4.21 .

4.3.1. Geometric preliminaries. We remind the reader our convention that we denote the squares in a Mondrian tableau in the math font ( $\operatorname{such}$ as $S$ ) and the corresponding vector spaces in the Roman font (such as $\mathrm{S}$ ). In a painted Mondrian tableau a square of side-length $s$ represents a vector space of dimension $s$. The red squares denote the constraints on the subspaces $V_{1}$ and the black squares denote the constraints on the vector spaces $V_{2}$.

Definition 4.42. Let $M$ be a painted Mondrian tableau for $F\left(k_{1}, k_{2} ; n\right)$. Let $\Sigma_{M}$ be the subvariety of $F\left(k_{1}, k_{2} ; n\right)$ defined as the closure of the locus of pairs $\left(V_{1} \subset V_{2}\right)$ that satisfy the following properties.

(1) For every black square $B_{i} \in M, \operatorname{dim}\left(V_{2} \cap \mathrm{B}_{i}\right)=\#_{B} B_{i}(M)$. For any pair of black squares $B_{i}, B_{j} \in M$, $\operatorname{dim}\left(V_{2} \cap \mathrm{B}_{i} \cap \mathrm{B}_{j}\right)=\#_{B}\left(B_{i} \cap B_{j}\right)(M)$.

(2) For any red square $R_{i} \in M$, $\operatorname{dim}\left(V_{1} \cap \mathrm{R}_{i}\right)=\#_{R} R_{i}(M)$. For any pair of red squares $R_{i}, R_{j} \in M$, $\operatorname{dim}\left(V_{1} \cap \mathrm{R}_{i} \cap \mathrm{R}_{j}\right)=\#_{R}\left(R_{i} \cap R_{j}\right)(M)$. Furthermore, the subspace of $V_{1}$ contained in $\mathrm{R}_{i}$ is contained in the subspace of $V_{2}$ spanned by $\left(V_{2} \cap \mathrm{B}_{j}\right)$ for $B_{j} \subset R_{i}$.

$\Sigma_{M}$ is an irreducible subvariety of $F\left(k_{1}, k_{2} ; n\right)$ of dimension equal to the dimension of $M$.

Proposition 4.43. Let $M$ be a painted Mondrian tableau for $F\left(k_{1}, k_{2} ; n\right)$. The variety $\Sigma_{M}$ associated to $M$ is an irreducible subvariety of $F\left(k_{1}, k_{2} ; n\right)$ of dimension

$$
\sum_{i=1}^{k_{2}}\left|B_{i}\right|+\sum_{i=1}^{k_{1}} \#_{B} R_{i}(M)-\sum_{i=1}^{k_{2}} \#_{B} B_{i}(M)-\sum_{i=1}^{k_{1}} \#_{R} R_{i}(M) .
$$

Proof. In order to prove the proposition we construct an the open subset of $\Sigma_{M}$ used in the definition as a sequence of open subsets of a Grassmannian bundle over an irreducible subvariety of $G\left(k_{2}, n\right)$. Consider the natural projection

$$
\pi_{2}: F\left(k_{1}, k_{2} ; n\right) \rightarrow G\left(k_{2}, n\right)
$$

that sends the pair $\left(V_{1}, V_{2}\right)$ to $V_{2}$. The image of $\Sigma_{M}$ under this projection is easy to describe. It is given by the variety associated to the generalized Mondrian tableau consisting of the black squares of $M$. Since the black squares of $M$ form a generalized Mondrian tableau, the projection is an irreducible variety of dimension

$$
\sum_{i=1}^{k_{2}}\left|B_{i}\right|-\sum_{i=1}^{k_{2}} \#_{B} B_{i}(M)
$$

On the other hand, the fiber over a point in the image of $\pi_{2}$ corresponds to choices of a $k_{1}$-dimensional subspaces that intersect any vector space $\mathrm{S}$ corresponding to a square $S$ in dimension equal to $\#_{R}(S)$ and is contained in $V_{2} \cap \mathrm{S}$. This is an open subset in a tower of Grassmannian bundles of dimension

$$
\sum_{i=1}^{k_{1}} \#_{B} R_{i}(M)-\sum_{i=1}^{k_{1}} \#_{R} R_{i}(M) \text {. }
$$

The proposition follows. 
Let $M$ be a painted Mondrian tableau associated to a Schubert variety $\Sigma_{\lambda}$ or to the initial tableau corresponding to the intersection of two Schubert varieties $\Sigma_{\lambda} \cap \Sigma_{\mu}$. Then the variety associated to $M$ is $\Sigma_{\lambda}$ or $\Sigma_{\lambda} \cap \Sigma_{\mu}$, respectively. These varieties (if non-empty) are all irreducible. Note that the intersection of two Schubert varieties is empty if and only if the corresponding Mondrian tableau is empty. Hence, we can assume that the intersection is non-empty. An open subset of $\Sigma_{M}$ is contained in $\Sigma_{\lambda}$ or $\Sigma_{\lambda} \cap \Sigma_{\mu}$, respectively. Hence it suffices to check that they have the same dimension. This is the content of Lemmas 4.13 and 4.14.

4.3.2. Degenerations. In this section let $M$ be a normalized, admissible painted Mondrian tableau. Suppose that $M$ is not nested. Let $S$ be the square of $M$ determined by Rule 4.15. Suppose that the lower-left chop of $S$ is $l c h(S)=\left\{e_{i}, \ldots, e_{j}\right\}$. As usual, we interpret the move on Mondrian tableaux as the degeneration $t e_{i}+(1-t) e_{j+1}$. Corresponding to this degeneration there is a flat family of varieties $\Sigma_{M}(t)$, where $\Sigma_{M}(1)$ represents the variety associated to $M$. We would like to describe the geometry of the flat limit $\Sigma_{M}(0)$.

The support of the limit $\Sigma_{M}(0)$ is determined by requiring the pair $\left(V_{1}, V_{2}\right)$ to satisfy the obvious rank conditions that they need to satisfy in the limit. We now make this precise in the next observation.

Observation 4.44. Let $\mathrm{B}_{i_{1}, \ldots, i_{j}}(t)$ denote the span of the vector spaces $\mathrm{B}_{i_{1}}(t), \ldots, \mathrm{B}_{i_{j}}(t)$ for $t \neq 0$. Let $\mathrm{B}_{i_{1}, \ldots, i_{j}}(0)$ denote the limit of the spans $\mathrm{B}_{i_{1}, \ldots, i_{j}}(t)$. Note that the limit of the spans need not equal the span of the limits. As noted in Observation 3.31 it is equal to the span of the limits unless one of the squares contains $e_{i}$, one of the squares contains $e_{j+1}$ and none of the squares contain both. In the latter case it is the span of the limit of the spans and $e_{i}$. Similarly, let $\mathrm{R}_{i_{1}, \ldots, i_{j}}(t)$ denote the span of $\mathrm{R}_{i_{1}}(t), \ldots, \mathrm{R}_{i_{j}}(t)$ for $t \neq 0$. Let $\mathrm{R}_{i_{1}, \ldots, i_{j}}(0)$ denote the limit of the spans. The same considerations apply to these vector spaces.

We note that the vector space $\mathrm{B}_{i_{1}, \ldots, i_{j}}(t)$ intersects the vector spaces $V_{2}$ parameterized by $\Sigma_{M}(t)$ in dimension at least $\#_{B}\left(B_{i_{1}, \ldots, i_{j}}(1)\right)(M)$. Since intersecting a vector space in a given dimension is a closed condition, vector spaces $V_{2}$ parameterized by the flat limit $\Sigma_{M}(0)$ have to intersect $\mathrm{B}_{i_{1}, \ldots, i_{j}}(0)$ in at least the same dimension. The same holds with $V_{2}$ replaced by $V_{1}$ and the black squares replaced by red squares. Furthermore, if the subspace $W$ of $V_{2}$ spanned by the subspaces of $V_{2}$ contained in $\mathrm{B}_{i_{1}}(1), \ldots, \mathrm{B}_{i_{j}}(1)$ intersects $V_{1}$ in dimension at least $h$, then the limit of $W$ has to intersect the limit of $V_{1}$ also in dimension at least $h$.

We can phrase this observation in terms of a slight generalization of painted Mondrian tableaux.

We will shortly see that this simple geometric observation is sufficient to determine the set theoretic limits of the degeneration.

Theorem 4.45 (The geometric Littlewood-Richardson Rule). The support of the flat limit $\Sigma_{M}(0)$ is equal to the union of the varieties associated to the painted Mondrian tableaux described in Algorithm 4.18. Furthermore, $\Sigma_{M}(0)$ is generically reduced along each of these varieties.

We first show that Theorem 4.45 implies Theorem 4.21.

Proof of Theorem 4.21. The variety associated to the initial tableau $M\left(\sigma_{\lambda}^{\delta}, \sigma_{\mu}^{\kappa}\right)$ is the intersection of the two Schubert varieties $\Sigma_{\lambda}^{\delta}$ and $\Sigma_{\mu}^{\kappa}$. By Lemma 4.11 the initial tableau is a normalized, admissible painted Mondrian tableau. By Proposition 4.41 the Algorithm 4.19 transforms normalized, admissible, painted Mondrian tableaux to normalized, admissible, painted Mondrian tableaux and terminates in a collection of tableaux associated to Schubert varieties. By Theorem 4.45 the class of the variety associated to a tableau is the sum of the classes of the varieties associated to the tableau at the end of the algorithm. Hence we obtain a Littlewood-Richardson rule for two-step flag varieties.

We will now prove Theorem 4.45. The proof will be very similar to the proof of 3.32. Using Observation 4.44 we will impose conditions on the supports of the irreducible components of $\Sigma_{M}(0)$ that can be the supports of Weil divisors of the family $\Sigma_{M}(t)$. First, a dimension count will show that the loci that satisfy all the constraints have dimension at most the dimension of $\Sigma_{M}(1)$. Among these loci we will determine the ones that have the same dimension as $\Sigma_{M}(1)$. These are the only loci that can support an irreducible component of $\Sigma_{M}(0)$ of dimension equal to $\Sigma_{M}(1)$. Once we know that $\Sigma_{M}(0)$ is supported along varieties associated to tableaux described in Algorithm 4.18, a very simple tangent space calculation shows that $\Sigma_{M}(0)$ is generically reduced along each irreducible component. In fact, this calculation reduces to the three examples described in 
the introduction and is simply the statement that when the cycles are in the specialized position the intersection of the corresponding varieties in $F\left(k_{1}, k_{2} ; n\right)$ is still generically transverse.

Proof of Theorem 4.45. Let $M^{\prime}$ denote the tableau obtained by moving $S$ according to Rule 4.16. Let $\Sigma_{M}(t)$ be the flat family corresponding to the move. Let $Y$ be a maximal irreducible component of the flat limit $\Sigma_{M}(0)$. Suppose that for a general pair $\left(V_{1}, V_{2}\right)$ of linear spaces parameterized by $Y$, the subspace of $V_{2}$ contained in each of $\mathrm{B}_{i}(0)$ has dimension $\#_{B} B_{i}(M)$ and $\operatorname{dim}\left(V_{2} \cap \mathrm{B}_{i}(0) \cap \mathrm{B}_{j}(0)\right)=\#_{B}\left(B_{i} \cap B_{j}\right)$. Furthermore, suppose that the subspace of $V_{1}$ contained in each of the $\mathrm{R}_{i}(0)$ has dimension $\#{ }_{R} R_{i}(M)$ and $\operatorname{dim}\left(V_{1} \cap \mathrm{R}_{i}(0) \cap \mathrm{R}_{j}(0)\right)=\# R_{R}\left(R_{i} \cap R_{j}\right)$. Then by Observation 4.44, $Y$ has to be contained in the variety associated to the tableau $M^{\prime \prime}$ obtained from $M^{\prime}$ by shrinking the red squares of $M^{\prime}$ so that they are the spans of the black squares contained in them. The proof of Lemma 3.28 carries over to show that the variety associated to $M^{\prime \prime}$ and to its normalization $M_{0}$ are equal. Hence, we conclude that $Y$ has to be contained in $\Sigma_{M_{0}}$. By Lemma 4.35, the dimension of $\Sigma_{M_{0}}$ is at most equal to the dimension of $\Sigma_{M}$. Hence, for there to be such a component, $\operatorname{dim} M_{0}=\operatorname{dim} M$. Since $Y$ and $\Sigma_{M_{0}}$ are both irreducible of the same dimension, we conclude that the support of $Y$ equals $\Sigma_{M_{0}}$.

Now suppose the largest black neighbor of $S$ satisfies $\left|N_{r}-S\right|=\#_{B}\left(N_{r} \cup S\right)(M)-\#{ }_{B} S(M)$. Then it is not possible for the limiting vector space $V_{2}$ to satisfy $\operatorname{dim}\left(V_{2} \cap \mathrm{B}_{\mathrm{i}}(0)\right)=\#_{B} B_{i}(M)$ and $\operatorname{dim}\left(V_{2} \cap \mathrm{B}_{i}(0) \cap\right.$ $\left.\mathrm{B}_{j}(0)\right)=\#_{B}\left(B_{i} \cap B_{j}\right)(M)$ for all black squares $B_{i}, B_{j}$. However, for a general point of an irreducible component $Y \subset \Sigma_{M}(0)$, the limiting positions of the subspaces of $V_{1}$ and $V_{2}$ contained in $R_{i}(t)$ and $R_{j}(t)$ and $B_{i}(t)$ and $B_{j}(t)$, respectively, may remain independent for all the squares in $M$. Similarly, if $S(0)$ contains the smallest neighbor $N_{1}$ of $S$, then it is not possible for $V_{2}$ to intersect $\mathrm{S}(0)$ in dimension equal to \# $B(M)$ unless the subspace of $V_{2}$ contained in $S(0)$ becomes dependent with the subspace of $V_{2}$ contained in $N_{1}(0)$. However, there may be components $Y$ of $\Sigma_{M}(0)$, where the limits of the subspaces $V_{1}$ and $V_{2}$ contained in $R_{i}(t)$ and $R_{j}(t)$ and $B_{i}(t)$ and $B_{j}(t)$ for every red and black square in $M$ remain independent in the limit. In these cases by Observation 4.44, the irreducible component $Y$ of $\Sigma_{M}(0)$ has to be contained in the variety associated to the normalization of the tableau $M^{\prime \prime}$ obtained by shrinking the red squares in $M^{\prime}$ to be the spans of the black squares contained in them. By Lemma 4.35 this normalization has at most the dimension of $M$. Hence this case can occur only when the dimension of $M_{0}$ equals the dimension of $M$. Since both $Y$ and $\Sigma_{M_{0}}$ are irreducible of the same dimension, we conclude that the support of $Y$ has to be $\Sigma_{M_{0}}$.

Let $Y$ be a maximal irreducible component of $\Sigma_{M}(0)$ whose support is not $\Sigma_{M_{0}}$. Now we can assume that in a general pair of linear spaces $\left(V_{1}, V_{2}\right)$ parameterized by $Y$ the limits of the subspaces of $V_{1}$ or $V_{2}$ contained in at least one pair of vector spaces $\mathrm{R}_{i}(t)$ and $\mathrm{R}_{j}(t)$ or $\mathrm{B}_{i}(t)$ and $\mathrm{B}_{j}(t)$ become dependent. Using Observation 4.44 we can build a variety corresponding to a painted Mondrian tableau that contains the support of $Y$. Suppose first that at a general point of $Y$, the subspaces of $V_{1}$ contained in $\mathrm{R}_{i}(0)$ and $\mathrm{R}_{j}(0)$ remain independent. There are two possible cases.

Case I: At a general point $\left(V_{1}, V_{2}\right)$ parameterized by $Y$, the subspace of $V_{2}$ intersects $\mathrm{R}_{i}(0)$ in an \# $R_{i}(M)$ dimensional subspace of $V_{2}$ for every vector space $\mathrm{R}_{i}(0)$ represented by a red square $R_{i}(0)$.

Case II: At a general point $\left(V_{1}, V_{2}\right)$ parameterized by $Y$, the subspace of $V_{2}$ intersects $\mathrm{R}_{i}(0)$ for some red square in dimension larger than $\#_{B} R_{i}(M)$.

In Corollary 3.44 we have verified that any $k_{2}$-plane parameterized by the limit $\Sigma_{M}(0)$ must be contained in one of the three varieties described by Corollary 3.44. These have at most the dimension equal to the dimension of the projection of $\Sigma_{M}$ to $G\left(k_{2}, n\right)$. If the generic fiber dimension of the projection of $Y$ to $G\left(k_{2}, n\right)$ is less than the dimension of the generic fiber dimension of the projection of $\Sigma_{M}$ to $G\left(k_{2}, n\right)$, then the dimension of $Y$ is strictly less than the dimension of $\Sigma_{M}$. Consequently $Y$ cannot support a component of the flat limit $\Sigma_{M}(0)$.

In Case I, the generic fiber dimension of the projection of $Y$ to $G\left(k_{2}, n\right)$ is equal to the generic fiber dimension of the projection of $\Sigma_{M}$ to $G\left(k_{2}, n\right)$. Consequently, the dimension of the projection of $Y$ to $G\left(k_{2}, n\right)$ has to have the same dimension as the projection of $\Sigma_{M}$ to $G\left(k_{2}, n\right)$. By Corollary 3.44 the projection of $Y$ to $G\left(k_{2}, n\right)$ is the variety associated to the tableau formed by the black squares of one of $M_{2}\left(N_{i}\right)$ where $N_{i}$ is a black neighbor of $S$ (assuming $S$ does have any black neighbors). Recall that we are assuming that $Y$ is not supported along $\Sigma_{M_{0}}$. Hence the projection of $Y$ is not supported along the variety associated to the black squares of $\Sigma_{M_{0}}$. Otherwise, by Observation 4.44 the support of $Y$ would be $\Sigma_{M_{0}}$. By Observation 4.44 each subspace of $V_{1}$ contained in $\mathrm{R}_{i}(0)$ is also contained in the span of $V_{2} \cap \mathrm{R}_{i}(0)$. Hence $Y$ has to be contained in the variety associated to the tableau consisting of the black squares of $M_{2}\left(N_{i}\right)$ and the red squares $R_{i}$ of $M^{\prime}$ that have been shrunk so that 
they are the spans of the black squares contained in them. Finally, normalizing this tableau does not change the variety associated to it. Only the red squares need to be normalized. Suppose $R_{1} \subset R_{2}$ have the same lower-left corner. Let $B_{1} \subset R_{2}$ be the black square whose lower-left corner is southwest most among the black squares $B_{i}$ with $l\left(B_{i}\right)>l\left(R_{2}\right)$. Shrinking $R_{2}$ so that $l\left(R_{2}\right)=l\left(B_{1}\right)$ does not change the variety by the argument given for Lemma 3.28. We conclude that the support of $Y$ has to be $\Sigma_{M_{2}\left(N_{i}\right)}$.

In case II, let $R_{0}(0)$ be the square with lower-left most corner such that $\mathrm{R}_{0}(0)$ has a larger dimensional intersection with $V_{2}$ than $\#_{B} R_{0}(M)$. Let $\mathrm{W}$ be the vector space spanned by the basis elements northeast of (and including) $l\left(R_{0}(0)\right)$. This case splits to a few cases.

Case A There exists a black square $B^{\prime}(0) \notin R_{0}(0)$ with lower-left corner southwest of $R_{0}(0)$ such that the dimension of $V_{2} \cap \mathrm{B}^{\prime}(0) \cap \mathrm{R}_{i}(0)$ is greater than $\#_{B}\left(B^{\prime}(0) \cap R_{0}(0)\right)(M)$. Suppose $B^{\prime}$ is the minimal square (with respect to inclusion) among such squares. This case splits to two cases.

i Either the dimension of $V_{2} \cap \mathrm{W}=\#_{B} W(M)$; or

ii The dimension of $V_{2} \cap \mathrm{W}>\#_{B} W(M)$.

Case B There does not exist a black square $B_{j}(0) \notin R_{0}(0)$ with lower-left corner southwest of $R_{0}(0)$ such that the dimension of $V_{2} \cap \mathrm{B}_{\mathrm{j}}(0) \cap \mathrm{R}_{i}(0)$ is greater than $\#_{B}\left(B_{j}(0) \cap R_{0}(0)\right)(M)$. In that case let $B_{j}(0)$ be the smallest square (with respect to inclusion) such that $V_{2} \cap \mathrm{B}_{\mathrm{j}}(0) \cap \mathrm{R}_{i}(0)$ is greater than $\#_{B}\left(B_{j}(0) \cap R_{0}(0)\right)(M)$.

We will show that Case A ii and Case B are not possible unless $Y$ is supported along $\Sigma_{M_{0}}$. In that case $B^{\prime}(0)$ is either $S(0)$ or the smallest neighbor of $S$ and $R_{0}(0)$ is the red neighbor of $S$ or $S(0)$, respectively. Before giving the argument we illustrate the dimension changes in some examples in Figure 19.

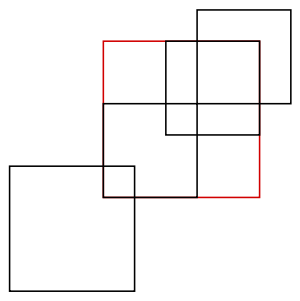

dimension 10

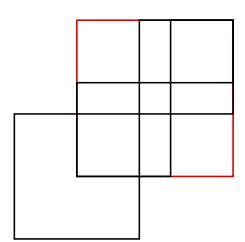

dimension 9

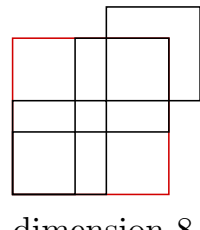

dimension 8

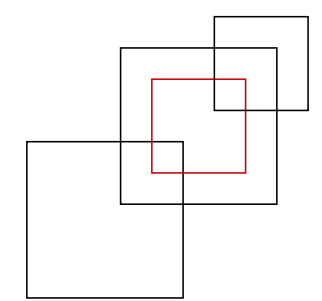

dimension 11

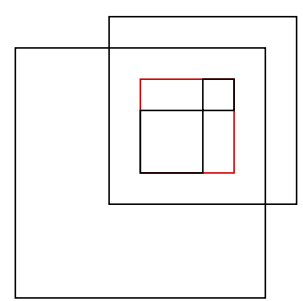

dimension 10

FigurE 19. Some sample varieties and how their dimensions change.

Let $T$ be the square corresponding to the smallest vector space spanned by $e_{1}, \ldots, e_{n}$ in the intersection of $B^{\prime}(0)$ and $R_{0}(0)$ for which $V_{2}$ intersects $\mathrm{R}_{i}(0)$ in dimension greater than $\#_{B} T\left(M^{\prime}\right)$. In Case $\mathrm{A}$ ii and Case $\mathrm{B}$ form a tableau of black squares $M^{\prime \prime}$ by replacing $B^{\prime}$ among the black squares of $M^{\prime}$ with $T$. Normalize the black squares. Form a tableau of red squares by taking the red squares of $M^{\prime}$. Declare that a black square $B$ is contained in a red square $R$ if there exists a black square $B^{\prime \prime}$ in $M^{\prime}$ containing $B$ which is contained in $R$. Denote the containment relations among black squares and red squares by $I$. Denote by $\#_{B}^{I} R\left(M^{\prime \prime}\right)$ the number of black squares in $M^{\prime \prime}$ contained in $R$ according to $I$. Then in Definition 4.42 we can replace containment relations among red and black squares by those that are in $I$ and the symbol $\#_{B} R$ by $\#_{B}^{I} R$ to obtain an irreducible subvariety of $F\left(k_{1}, k_{2} ; n\right)$ of dimension at most

$$
\sum_{i=1}^{k_{2}}\left|B_{i}\right|+\sum_{i=1}^{k_{1}} \#_{B}^{I} R_{i}\left(M^{\prime \prime}\right)-\sum_{i=1}^{k_{2}} \#_{B} B_{i}\left(M^{\prime \prime}\right)-\sum_{i=1}^{k_{1}} \#_{R} R_{i}\left(M^{\prime}\right)
$$

The proof of Proposition 4.43 remains unchanged.

We can estimate how the dimension changes when we apply this procedure. First, let us see the change in dimension prior to normalizing the black squares. It is useful to consider the projection of $Y$ to $G\left(k_{2}, n\right)$. The fiber dimension of the projection increases by the number of red squares $r$ that did not contain $B^{\prime}$, but contain $T$ and have a black square containing $T$. On the other hand, the number of containment relations among 
black squares increases by at least $r$ due to the fact that $r$ of the black squares contained in the red squares and not containing $B(0)$ start containing the intersection. However, some of the black squares contained in $B^{\prime}$ may no longer be contained in $B$. Suppose there are $m$ of those. The difference in side-length between $B^{\prime}$ and $T$ is at least $r+m$. Since $M$ is normalized, it can only be $r+m$ if $B^{\prime}=S(0)$ and every vector $e_{i}$ with $l(S(0)) \leq e_{i}<R_{0}(0)$ is the lower-left corner of a square strictly contained in $S(0)$; or $B^{\prime}$ is the smallest neighbor of $S$ and is contained in $S(0)$. For every other instance, the dimension of the projection strictly decreases. Note that normalizing the black squares cannot increase the dimension. We conclude that in this case we either obtain the Tableau $M_{0}$ in the cases described or the dimension of the variety strictly decreases by this procedure.

In Case A i, Using Observation 4.44, we can build a variety that must contain the support of $Y$. Replace the square $B^{\prime}(0)$ with the square $T$ corresponding to the smallest vector space spanned by $e_{1}, \ldots, e_{n}$ in the intersection of $B^{\prime}(0)$ and $R_{0}(0)$ for which $V_{2}$ intersects $\mathrm{R}_{i}(0)$ in dimension greater than $\#_{B} T\left(M^{\prime}\right)$. In this case $\operatorname{dim}\left(V_{2} \cap \mathrm{W}\right)=\#_{B} W(M)$. Let $B_{1}$ be the square in $M^{\prime}$ whose lower-left corner is southwest most among the squares not contained in $R_{0}(0)$. If $B_{1}$ coincides with a red square $R_{1}$, let $B_{2}$ be the square whose lower-left corner is southwest most among the squares not contained in $R_{1}$. Inductively if $B_{h}$ coincides with a red square $R_{h}$, let $B_{h+1}$ be the square whose lower-left corner is southwest most among the squares not contained in $R_{h}$. Stop if $B_{l}$ does not coincide with a red square. Let $B_{1}, \ldots, B_{l}$ be these black squares. Let $X$ be the smallest black square contained in $R_{0}(0)$ with $l(X)<l\left(B_{1}\right)$ and which agrees with $R_{0}(0)$ northeast of $l(X)$. If there are any black squares that contain the squares $R_{0}, \ldots, R_{l-1}$, but not $B^{\prime}$ or $B_{l}$, list the minimal ones with respect to inclusion $A_{1}, \ldots, A_{r}$ ordered by their lower-left corners. Form the collection of black squares $M^{\prime \prime}$ by replacing the black squares $B^{\prime}, B_{1}, \ldots, B_{l}, A_{1}, \ldots, A_{r}$ in $M^{\prime}$ with $T, B_{1} \cap X, B_{1} \cap B_{2}, \ldots, B_{h} \cap B_{h+1}, \ldots B_{l-1} \cap B_{l}$ and the span of $B^{\prime}$ and $A_{1}, A_{i}$ and $A_{i+1}$ for $i=1, \ldots, r-1$ and the span of $A_{r}$ and $B_{l}$. Note that $V_{2}$ has to intersect $\mathrm{B}_{h} \cap \mathrm{B}_{h+1}$ in dimension at least the number of squares contained in $M^{\prime \prime}$. We are assuming that $\operatorname{dim}\left(V_{2} \cap \mathrm{W}\right)=\#_{B}(W)$, hence the subspace of $V_{2}$ contained in the span $\mathrm{W}_{1}$ of basis vectors northeast of $B_{1}$ has to intersect the subspace of $V_{2}$ contained in $R_{0}(0)$ in dimension larger than $\#_{B}\left(W_{1} \cap R_{0}(0)\right)$, hence $V_{2}$ must intersect $\mathrm{B}_{1} \cap \mathrm{R}_{i}(0)$. In particular, $V_{2}$ must intersect $\mathrm{X} \cap \mathrm{R}_{i}(0)$. If $B_{1}$ coincides with a red square, note that by APM1 and APM2 for $M^{\prime}$ any square strictly contained in $B_{1}$ is also contained in $R_{0}(0)$. Either $V_{2}$ intersects the span of $\mathrm{R}_{i}(0)$ and $\mathrm{B}_{1}$ in dimension $\#_{B} R_{0}(0)\left(M^{\prime}\right)+1$, but since $V_{2}$ already intersects $\mathrm{R}_{i}(0)$ in dimension $\#_{B} R_{0}(0)\left(M^{\prime}\right)+1$, we would have $V_{1}$ intersects $\mathrm{R}_{1} \cap \mathrm{R}_{i}(0)$ contrary to our assumption. Else $V_{2}$ intersects $\mathrm{B}_{2} \cap \mathrm{B}_{1}$. Continuing by induction we see that $V_{2}$ has to intersect $\mathrm{B}_{h} \cap \mathrm{B}_{h+1}$ in dimension at least the number of squares contained in $M^{\prime \prime}$. If any of the intersections are empty, then clearly this locus cannot exist. Similarly, by Observation $3.31 V_{2}$ has to intersect the limits of the spans of the consecutive vector spaces among $\mathrm{B}^{\prime}, \mathrm{A}_{i}$ and $\mathrm{B}_{l}$ as claimed. Next, form the collection of red squares consisting of the red squares in $M^{\prime}$. Finally, specify inclusions $I$ of black squares in $M^{\prime \prime}$ into red squares where a black square $B^{\prime \prime}$ is included in a red square $R^{\prime}$ if in $M^{\prime}$ there exists a black square in $M^{\prime}$ containing $B^{\prime \prime}$ and contained in $R^{\prime}$. As in the previous case, we can associate an irreducible subvariety of $F\left(k_{1}, k_{2} ; n\right)$ to this new set of data as in Definition 4.42.

We can calculate how this procedure changes the dimension. Let $r$ be the number of squares that start containing a black square $B$, which they previously did not contain, in one of their black squares. The fiber dimension of the projection to $G\left(k_{2}, n\right)$ increases by $r$. The dimension of the image changes as follows: By Corollary 3.44 the dimension decreases by at least $r+1$ if $B^{\prime}$ is not $S(0)$ and by at least $r$ if $B^{\prime}$ is $S(0)$. Hence, in order, to have a variety that can support $Y, B^{\prime}$ must be $S(0)$. Furthermore, $R_{0}(0)$ must be a red neighbor of $S(0)$. In all other instances the dimension decreases by at least one. Now we can explicitly describe the change in dimension. The total side-lengths of the black squares increases by 1 . Since there is no containment relations among the red squares for which the construction takes place, the fiber dimension increases by at most $j-i+1$. The containment relations among the black squares changes as follows: Suppose $S$ has two black neighbors. Then by APM2 the larger black neighbor contains $R_{0}(0)$ and the black square that forms the final intersection with $R_{l}(0)$ cannot be contained in the larger black neighbor of $S$ since $F$ does not contain any fillers and $B_{l}$ does not coincide with a red square. Hence the total containment relations among the black squares increases by at least $j-i+3$. One for each of $S(0)$, the last black square and the black squares coinciding with the red squares and the larger black neighbor. Hence, this possibility cannot occur if $S$ has more than one black neighbor. Similarly, if the red neighbor of $S$ does not contain the black neighbor of $S$, the procedure strictly decreases the dimension by a similar argument. Finally, suppose that $S$ has one black neighbor, but this neighbor does not coincide with the red square. By the same reasoning this increases the number of containment relations among 
the black squares to at least $j-i+3$. We conclude that the procedure strictly decreases the dimension unless $S$ has a unique black member that coincides with a red square.

We can by repeatedly applying the two procedures described above build an irreducible subvariety of $F\left(k_{1}, k_{2} ; n\right)$ that contains the irreducible component $Y$. Applying the procedure potentially forces us to leave the category of painted Mondrian tableaux. However, note that the procedures only involve the black squares. Furthermore, each repetition of the procedure strictly decreases the dimension of the resulting variety unless the total side-length of the black squares increase by at least one. Since this can happen at most once we conclude that the only possibilities are as follows: Either we are in case A i; $B^{\prime}$ is $S(0) ; S$ has a unique black neighbor that coincides with a red square. Or we are in case A ii; $B^{\prime}$ is $S(0)$; and every vector $e_{i}$ with $l(S(0)) \leq e_{i}<R_{0}(0)$ is the lower-left corner of a square strictly contained in $S(0)$. Or we are in case $\mathrm{B} ; B^{\prime}$ is the smallest neighbor of $S(0) ; R_{0}(0)$ coincides with $S(0)$ and contains $B^{\prime}$. We can also describe the corresponding tableaux. We already saw that in cases A ii and B we obtain the Tableau $M_{0}$. In Case A i, the procedure produces the tableau $M_{3}(N R)$ (prior normalizing and shrinking the red squares to become the span of black squares contained in them). A priori at a general point of $Y$ the subspaces of $V_{2}$ contained in the vector spaces corresponding to the squares can become more dependent. However, by Corollary 3.44 this strictly decreases the dimension of the image of the projection. Since we are assuming that the support of $Y$ is not $\Sigma_{M_{0}}$, we conclude that $Y$ has to be supported on $\Sigma_{M_{3}(N R)}$.

Next, we can assume that at a general point of a maximal irreducible component $Y$ of $\Sigma_{M}(0)$, the subspaces of $V_{1}$ intersect $\mathrm{R}_{i}(0) \cap \mathrm{R}_{j}(0)$ in dimension greater than $\#_{R}\left(R_{i} \cap R_{j}\right)(M)$. Then using Observation 4.44, we can build a subvariety of $F\left(k_{1}, k_{2} ; n\right)$ that has to contain $Y$. Consider the smallest subspace $\mathrm{T}$ such that $V_{1}$ at a general point of $Y$ intersects $\mathrm{T}$ in dimension greater than the number of red squares contained in $T$. If $T$ is not contained in the span of black squares we add a black square coinciding with $T$. We then adjust the tableau consisting of red squares as in Corollary 3.44 and the tableau consisting of black squares as in Corollary 3.44. That is we list the minimal red squares $R_{1}, \ldots, R_{l}$ and the minimal black squares $B_{1}, \ldots, B_{m}$ containing $T$ and apply the procedure in Corollary 3.44

We continue until $V_{1}$ intersects the vector spaces corresponding to the intersection of any two red squares in dimension equal to the number of red squares contained in that intersection. Call the resulting tableau $\tilde{M}$. If for each red square $R$, the dimension of the subspace of $V_{2}$ contained in $\mathrm{R}$ is equal to $\#_{B} R(\tilde{M})$ and for any two $B_{1}, B_{2}$ in $\tilde{M}, V_{2}$ intersects $\mathrm{B}_{1} \cap \mathrm{B}_{2}$ in dimension equal to $\#_{B}\left(B_{1} \cap B_{2}\right)(\tilde{M})$, then the support of $Y$ is contained in $\Sigma_{\tilde{M}}$. Otherwise, we have to run the procedure described in Case II. Running the procedure on Tableau $\tilde{M}$ cannot increase the dimension of the resulting tableau by an argument analogous to the one given after Case II above. Hence, we can estimate the dimension of $\Sigma_{\tilde{M}}$.

We can consider the first projection $\pi_{1}: Y \rightarrow G\left(k_{1}, n\right)$. Let $M_{R}$ be the tableau consisting of the red squares in $M$. By Corollary 3.44 the projection of $Y$ has to be contained in the variety associated to one of the tableau $M_{0}$ or $M_{1}(N)$ obtained from $M_{R}$ by Algorithm 3.40. Next we observe that forming the tableau $\tilde{M}$ preserves or decreases the fiber dimension of the projection to $G\left(k_{1}, n\right)$.

Let $A$ be a painted Mondrian tableau. Then the projection of the variety $\Sigma_{A}$ to $G\left(k_{1}, n\right)$ is obtained by taking the tableau consisting of the red squares of $A$. The fiber dimension of this projection is calculated by

$$
\sum_{B_{i} \in A}\left|B_{i}\right|-\sum_{B_{i} \in A} \#_{B} B_{i}(A)+\sum_{R_{i} \in A} \#_{B} R_{i}(A)-\sum_{R_{i} \in A}\left|R_{i}\right| .
$$

More generally, the fiber dimension of the projection is calculated by the dimension for the choice of $V_{2}$ containing $V_{1}$ and satisfying the constraints imposed by the black squares of the tableau. This is calculated as in Corollary 3.44. Suppose there are $m$ minimal black squares containing the intersection. If the one with southwest most corner is not $S(0)$, then the contribution to the fiber dimension from the choice of $V_{2}$ decreases by at least $m-1$. If the square with southwest most corner is $S(0)$, then the contribution to the fiber dimension decreases by at least $m-2$. On the other hand, the containment relations among the subspaces of $V_{2}$ and $V_{1}$ can increase by the number of black squares which were not contained in a red square, but are now contained in the consecutive spans of the red squares. By APM1 and APM2 for $M$, if a black square $B$ which was not contained in $R_{h}$ or $R_{h+1}$ is contained in the span $R_{h, h+1}$, then $B$ must be among the minimal black squares in the construction. Otherwise, $B$ would contain a red square strictly contained in it containing a minimal black square. Hence, the minimal red squares could not be $R_{1}, \ldots, R_{l}$. Hence by APM2 and APM3, the number of black squares 
contained in red squares increases by at most $m-1$. If the square with southwest most corner is $S(0)$, the dimension of $V_{1}$ contained in the span also increases by 1 . Hence, we conclude that under the construction the generic fiber dimension of the projection to $G\left(k_{1}, n\right)$ does not increase.

Since $Y$ has the same dimension as $\Sigma_{M}$, we conclude that the projection of $\Sigma_{\tilde{M}}$ to $G\left(k_{1}, n\right)$ must equal the dimension of the projection of $\Sigma_{M}$ to $G\left(k_{1}, n\right)$. Hence by Corollary 3.44 , the red squares of $\tilde{M}$ must be one of $M_{0}$ or $M_{1}$ associated to $M_{R}$. Since we are assuming that we are not in the case $M_{0}$, we must be in the case $M_{1}$. By the same argument as in Corollary 3.44, this projection has the same dimension only if the move increases the dimension of intersection of $R$ (or a chop of $R$ ) with a neighbor of $R$ (respectively, of a chop of $R$, where we define the neighbor of a chop of $R$ as in definition 3.10 with every occurrence of $R$ replaced by the chop of $R$ ). By Proposition 4.29 this only happens when $S$ coincides with a red square $R$ since in every other case there exists a red square to the lower-left of $l(S)$ that $R$ does not contain. By APM1 the move cannot increase the length of intersection of a red square (or a chop of it) with a neighbor. We conclude that the dimension of the projection of $Y$ can have the same dimension only when $S$ coincides with a red square. Since the generic fiber dimension of the projection to $G\left(k_{1}, n\right)$ has to be maximal, we conclude that $\tilde{M}$ is equal to $M_{1}(N R)$. Now it is clear that running the procedure after Case II or Case I, strictly decreases the dimension. We conclude that $Y$ has to be supported on $\Sigma_{M_{1}(N R)}$.

Finally, suppose $B_{j}$ does not coincide with a red square of $M$ and $R_{i}$ does not coincide with a black neighbor of $B_{j}$. A priori there could exist a maximal irreducible component $Y$ of $\Sigma_{M}(0)$, where at a general point $V_{1}$ intersects $\mathrm{B}_{j}(0) \cap \mathrm{R}_{i}(0)$ in dimension greater than $\#_{R}\left(B_{j}(0) \cap R_{i}(0)\right)(M)$. Such loci have dimension strictly less than the dimension of $\Sigma_{M}$. Hence, they cannot support maximal irreducible components of $\Sigma_{M}(0)$. The argument is very similar to the previous cases, so we leave it to the reader.

This concludes the first step of the proof. In order to conclude the proof we have to check that $\Sigma_{M}(0)$ is reduced along each irreducible component. Using the same reduction as in the proof of Theorem 3.32 , we can assume that $M$ consists of $S$ and the squares that whose intersections and spans are being taken. Namely consider the subspace of $\Sigma_{M(t)}$ contained in the vector spaces coinciding with the squares whose intersections and spans are formed. Over an Zariski-open dense subset intersecting every component of $\Sigma_{M}(0)$, the restriction of $V_{1}$ and $V_{2}$ gives a smooth morphism to a smaller two-step flag variety. The statement about reducedness follows from the smaller flag variety.

Suppose first that $S$ does not coincide with a red square and the largest neighbor of $S$ does not coincide with a red square. In this case, the projection $\pi_{2}: F\left(k_{1}, k_{2} ; n\right) \rightarrow G\left(k_{2}, n\right)$ exhibits an open subset of the family intersecting every component of $\Sigma_{M}(0)$ as an open set in a tower of projective bundles. In this case the fact that

$\Sigma_{M_{2}\left(N_{i}\right)}$ and $\Sigma_{M_{0}}$ have multiplicity one follows from Theorem 3.32 by pulling back via this morphism. More generally, the fact that the components $\Sigma_{M_{2}\left(N_{i}\right)}$ have multiplicity one follows from Theorem 3.32 by pulling back by the projection morphism. Now suppose $S$ coincides with a red square, but the largest neighbor of $S$ is not red. Then consider the projection $\pi_{1}: F\left(k_{1}, k_{2} ; n\right) \rightarrow G\left(k_{1}, n\right)$. This projection exhibits an open subset of the family intersecting $\Sigma_{M_{1}(N R)}$ as a tower of projective bundles over an open subset in the variety associated to the tableau consisting of the red squares. By Theorem 3.32, it follows that $\Sigma_{M_{1}(N R)}$ occurs with multiplicity one. More generally, by the same argument $\Sigma_{M_{1}(N R)}$ occurs with multiplicity one. Next, we need to check that in case the largest neighbor of $S$ is red $\Sigma_{M_{3}(N R)}$ occurs with multiplicity one. This case immediately reduces to the case when $M$ consists of a black square $S$ a red neighbor $R$ and a black neighbor $N$ of $R$. That the multiplicity is one in this case is an easy local calculation. Finally, observe that $M_{0}$ has multiplicity one in the remaining two cases: If $S$ is black and the largest red neighbor contains $S(0)$; or if $S$ coincides with a red square and it contains its only black neighbor. That $\Sigma_{M_{0}}$ has multiplicity one in these two cases is obvious either by an easy local calculation or by Monk's rule. This concludes the proof of the theorem.

\section{A quantum Littlewood-Richardson rule for Grassmannians}

In this section we obtain a quantum Littlewood-Richardson rule for Grassmannians $G(k, n)$ as a corollary to Theorem 4.21 .

Given a Mondrian tableau for $\sigma_{\lambda}$ in $G(k, n)$ and an integer $d \leq k$, we can associate to it a painted Mondrian tableau in $F(k-d, k+d ; n)$ as follows: The Mondrian tableau associated to the Schubert variety $\sigma_{\lambda}$ consists of $k$ nested squares. We take the largest $k-d$ squares (those of index $d+1, \ldots, k$ ) and color them in $C_{1}$. 
We color the remaining squares in $C_{2}$. Finally, we add $d$ squares of color $C_{2}$ at the largest available places in the flag defining the Mondrian tableau of $\sigma_{\lambda}$ (see Figure 20 for two examples). We call the resulting painted Mondrian tableau the quantum Mondrian tableau of degree $d$ associated to $\sigma_{\lambda}$. This tableau is none other than the painted Mondrian tableau associated to the special Schubert variety $X_{\lambda}^{(d)}$ of $F(k-d, k+d ; n)$ defined in $\S 2$.
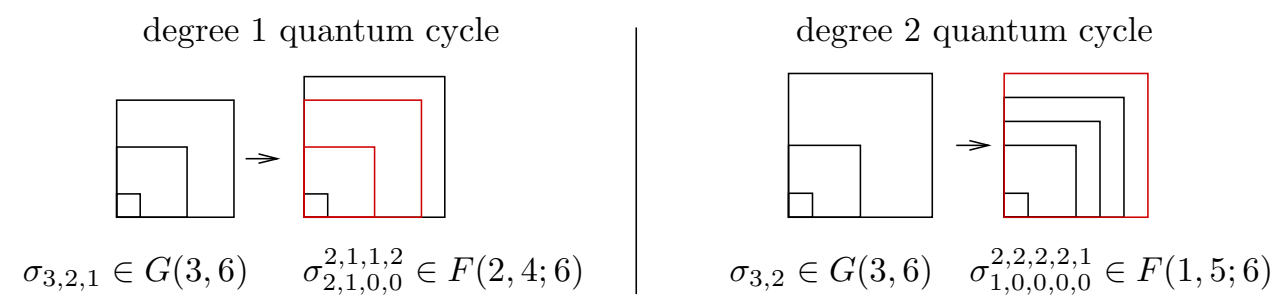

Figure 20. The quantum Mondrian tableaux associated to two Schubert varieties.

Let $\sigma_{\lambda}, \sigma_{\mu}$ and $\sigma_{\nu}$ be three Schubert cycles in $G(k, n)$ that satisfy the equality

$$
|\lambda|+|\mu|+|\nu|=k(n-k)+d n .
$$

Apply the algorithm described in the previous section to the quantum Mondrian tableau of degree $d$ associated to $\sigma_{\lambda}$ and $\sigma_{\mu}$ to express their intersections as a sum of Schubert cycles in $F(k-d, k+d ; n)$. Theorem 4.21 and Lemma 2.7 imply that the Gromov-Witten invariant $I_{d}\left(\sigma_{\lambda}, \sigma_{\mu}, \sigma_{\nu}\right)$ is equal to the number of times the Poincaré dual of the quantum Mondrian tableau of degree $d$ associated to $\sigma_{\nu}$ occurs in this product. We have obtained the following theorem.

Theorem 5.1. The three-pointed Gromov-Witten invariant $I_{d}\left(\sigma_{\lambda}, \sigma_{\mu}, \sigma_{\nu}\right)$ of $G(k, n)$ is equal to the number of times the Poincaré dual of the quantum Mondrian tableau of degree d associated to $\sigma_{\nu}$ occurs in the Algorithm 4.18 applied to the quantum Mondrian tableaux of degree $d$ associated to $\sigma_{\lambda}$ and $\sigma_{\mu}$ in an $n \times n$ square.

We illustrate the use of Theorem 5.1 by computing the Gromov-Witten invariant

$$
I_{G(3,6), d=1}\left(\sigma_{3,2,1}, \sigma_{3,2,1}, \sigma_{2,1}\right)=2 .
$$

Figure 21 demonstrates the computation. The quantum cycle of $d=1$ associated to $\sigma_{3,2,1}$ (respectively, $\sigma_{2,1}$ ) is $\sigma_{2,1,0,0}^{2,1,1,2}$ (respectively, $\sigma_{1,0,0,0}^{2,1,2,1}$ ). In order to calculate the Gromov-Witten invariant we have to find how many times $\sigma_{2,2,2,1}^{1,2,1,2}$ (the dual of $\sigma_{1,0,0,0}^{2,1,2,1}$ ) occurs in the square of the class $\sigma_{2,1,0,0}^{2,1,1,2}$. An easy calculation with painted Mondrian tableaux shows that the answer is 2 .

\section{REFERENCES}

[BS] N. Bergeron and F. Sottile. Schubert polynomials, the Bruhat order, and the geometry of flag manifolds. Duke Math. J. 95(1998), 373-423.

[BGG] I.N. Bernstein, I.M. Gelfand, and S.I. Gelfand. Schubert cells and the cohomology of the spaces G/P. Russian Math. Surveys 28:3(1973), 1-26.

[B] A. Bertram. Quantum Schubert calculus. Adv. Math. 128(1997), 289-305.

[Bu] A. S. Buch. Quantum cohomology of Grassmannians. Compositio Math. 137(2003), 227-235.

[BKT] A. S. Buch, A. Kresch, and H. Tamvakis. Gromov-Witten invariants on Grassmannians. J. Amer. Math. Soc. 16(2003), 901-915.

[C] I. Coskun. Degenerations of surface scrolls and the Gromov-Witten invariants of Grassmannians. J. Algebraic Geom. 15(2006), 223-284.

[CV] I. Coskun and R. Vakil. Geometric positivity in the cohomology of homogeneous spaces and generalized Schubert calculus. submitted.

[Ful1] W. Fulton. Young tableaux, volume 35 of London Mathematical Society Student Texts. Cambridge University Press, Cambridge, 1997.

[Ful2] W. Fulton. Intersection theory, volume 2 of Ergebnisse der Mathematik und ihrer Grenzgebiete. 3. Folge. A Series of Modern Surveys in Mathematics. Springer-Verlag, Berlin, second edition, 1998.

[FP] W. Fulton and R. Pandharipande. Notes on stable maps and quantum cohomology. In Algebraic geometry - Santa Cruz 1995, volume 62 Part 2 of Proc. Sympos. Pure Math., pages 45-96. Amer. Math. Soc., 1997. 


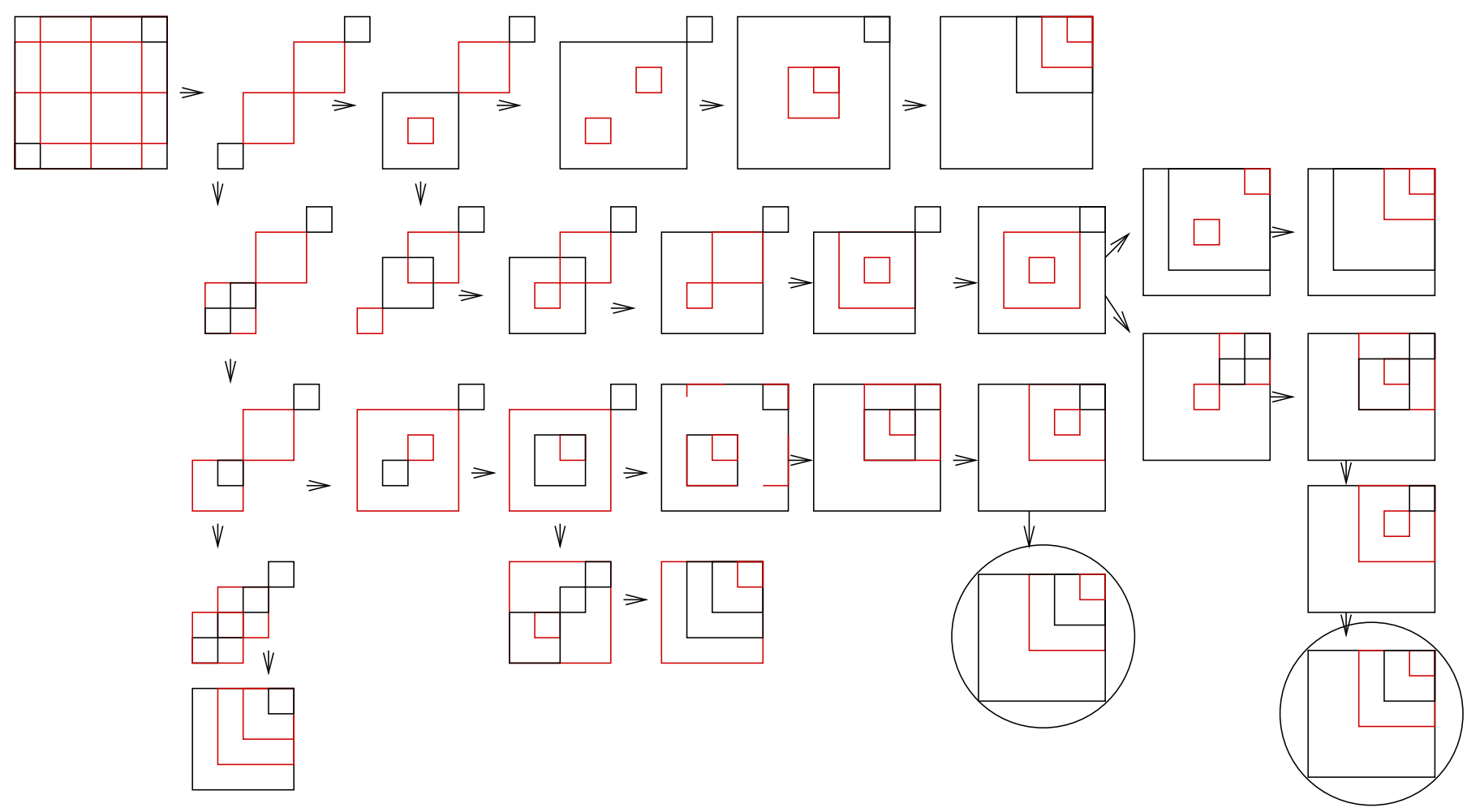

Figure 21. Computing the Gromov-Witten invariant $I_{G(3,6), d=1}\left(\sigma_{3,2,1}, \sigma_{3,2,1}, \sigma_{2,1}\right)=2$.

[FPi] W. Fulton and P. Pragacz. Schubert varieties and degeneracy loci, volume 1689 of Lecture Notes in Mathematics. SpringerVerlag, Berlin, 1998.

[GH] P. Griffiths and J. Harris. Principles of Algebraic Geometry. Wiley Interscience, 1978.

[Kl] S. L. Kleiman. The transversality of a general translate. Compositio Math. 28(1974), 287-297.

[KT] A. Knutson and T. Tao. Puzzles and (equivariant) cohomology of Grassmannians. Duke Math. J. 119(2003), 221-260.

$[\mathrm{KTW}]$ A. Knutson, T. Tao, and C. Woodward. The honeycomb model of $\mathrm{GL}_{n}(\mathbb{C})$ tensor products. II. Puzzles determine facets of the Littlewood-Richardson cone. J. Amer. Math. Soc. 17(2004), 19-48 (electronic).

[Kog] M. Kogan. RC-graphs and a generalized Littlewood-Richardson rule. Internat. Math. Res. Notices (2001), 765-782.

[V1] R. Vakil. A geometric Littlewood-Richardson rule. Ann. of Math. (2) 164(2006), 371-421. Appendix A written with A. Knutson.

[V2] R. Vakil. Schubert induction. Ann. of Math. (2) 164(2006), 489-512.

Massachusetts Institute of Technology, Mathematics Department, Cambridge, MA 02139

E-mail address: coskun@math.mit.edu 